University of Rhode Island

DigitalCommons@URI

Open Access Master's Theses

2017

\title{
Testbed for Trajectory Control of a Two-Wheeled Robot
}

Nathan Kando

University of Rhode Island, nate.kando@gmail.com

Follow this and additional works at: https://digitalcommons.uri.edu/theses

\section{Recommended Citation}

Kando, Nathan, "Testbed for Trajectory Control of a Two-Wheeled Robot" (2017). Open Access Master's Theses. Paper 1136.

https://digitalcommons.uri.edu/theses/1136

This Thesis is brought to you for free and open access by DigitalCommons@URI. It has been accepted for inclusion in Open Access Master's Theses by an authorized administrator of DigitalCommons@URI. For more information, please contact digitalcommons-group@uri.edu. 
TESTBED FOR TRAJECTORY CONTROL OF A TWO-WHEELED ROBOT

BY

NATHAN KANDO

A THESIS SUBMITTED IN PARTIAL FULFILLMENT OF THE REQUIREMENTS FOR THE DEGREE OF

MASTER OF SCIENCE

IN

ELECTRICAL ENGINEERING

UNIVERSITY OF RHODE ISLAND

2017 


\title{
MASTER OF SCIENCE THESIS
}

BY

NATHAN KANDO

APPROVED:

Thesis Committee:

\author{
Major Professor RICHARD J. VACCARO \\ PETER F. SWASZEK \\ ORLANDO MERINO \\ NASSER H. ZAWIA \\ DEAN OF THE GRADUATE SCHOOL
}




\begin{abstract}
This thesis develops a test platform for a control problem.

The inverted pendulum is selected as a well-established control problem. It is representative of an unstable nonlinear system which may remain balanced using any of several methods. Once balancing is achieved, multidimensional maneuvering is added as a supplemental control objective.

To approach the control problem hardware is selected which is then characterized and simulated, and then operated while communicating operational data.
\end{abstract}

The thesis provides a detailed description of the approaches to:

- Selecting the hardware.

- Characterizing the hardware.

- Developing a functioning controller.

- Simulating the results.

Additionally, a modular test platform is developed such that additional control approaches or characterization models could be implemented and hot-swapped. 


\section{Acknowledgements}

The research performed and described in this document could not have been completed without the insight and guidance of several parties.

First and foremost, I would like to acknowledge Professor Richard J. Vaccaro for his capabilities, his mentorship, and his professionalism in the STEAM fields community. The great level of knowledge which he has provided to me and countless others, whether in academic text, course lecture, personal conversation, or penned scrawl on scrap paper, is not to be underestimated.

Additional acknowledgement should be provided to Professor Joshua Hurst, who publicly released software drivers for the MinSeg hardware, and who extended the supported platforms of those drivers to make them accessible beyond their original use.

I would like to thank all other cited academic parties, particularly, [but in no particular order]:

- Yorihisa Yamamoto, for his two-wheeled robot dynamic model.

• "Phil O", Ryo Watanabe, and related peers, for their NXT motor studies.

- Mark Peltier, for his two-wheeled robot control publication

- Brian Howard and Linda Bushnell, for their MinSeg publication

Thank you for making your works available.

Finally, but of no less consideration, I would like to thank Holly Gaboriault who took photos on request, as well as the members of the Wikimedia Foundation, and the Stackexchange and Matlab Central communities, who offered their multidisciplinary expertise regarding any number of subjects, whether mathematical, technical, typographical, or otherwise, rapidly and on-demand. 


\section{Dedication}

For those who led by example

with an unyielding optimism and a devotion to the field which has only enriched;

for Laura and for Professor Richard Vaccaro.

Thank you for your own sizable accomplishments and for your guidance in mine. 


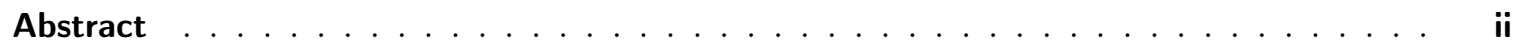

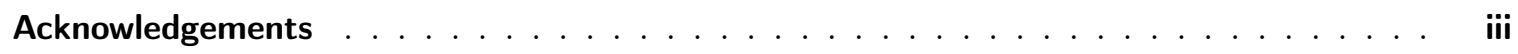

Dedication .......................... iv

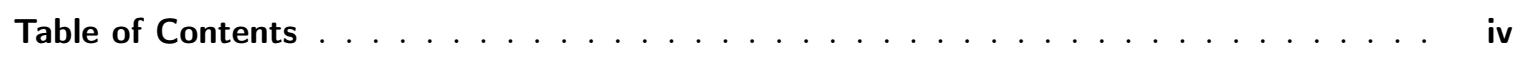

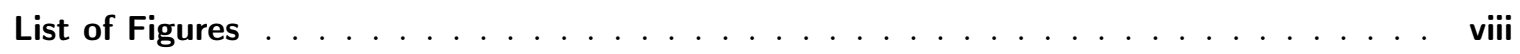

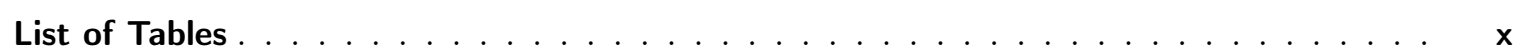

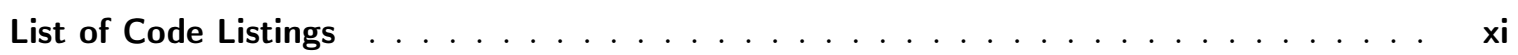

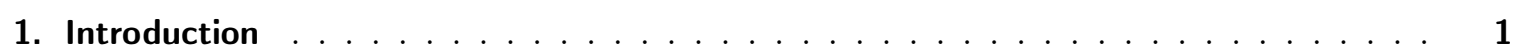

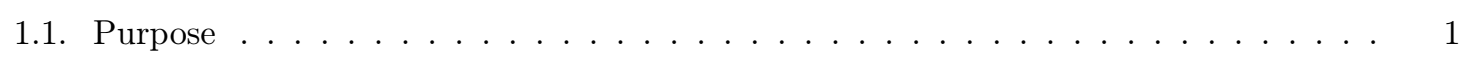

1.2. Statement of the Problem . . . . . . . . . . . . . . . 2

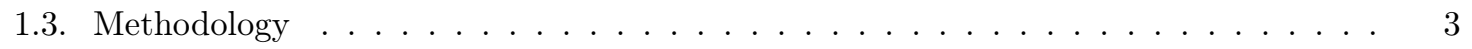

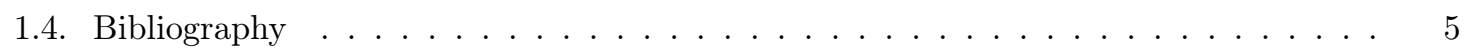

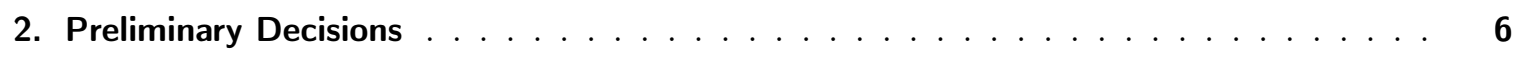

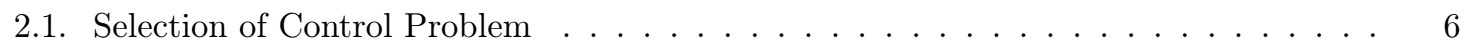

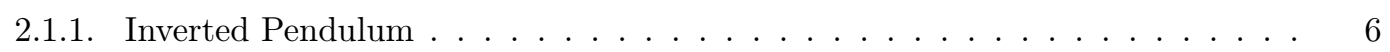

2.1.2. Two-Wheeled Robot . . . . . . . . . . . . . . . . . 7

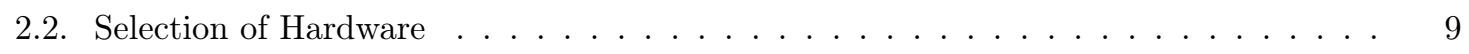

2.2.1. MinSeg M2V3 Two-Wheeled Robot . . . . . . . . . . . . . . . . . . 10

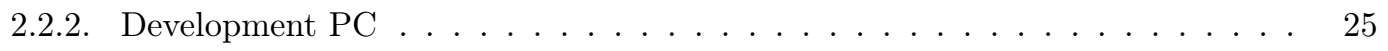

2.3. Selection of a Hardware Model . . . . . . . . . . . . . . . . . . . 28

2.4. Selection of Controller Design . . . . . . . . . . . . . . . . . . . 29

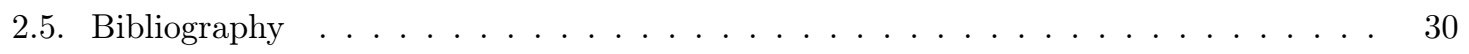

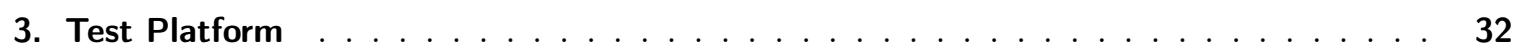

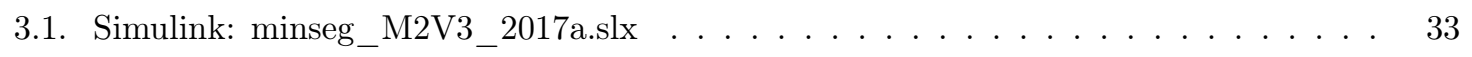

3.1.1. Root . . . . . . . . . . . . . . . . 33

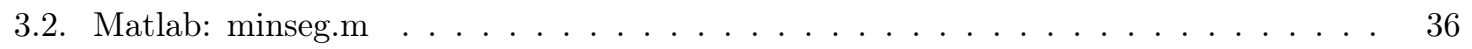




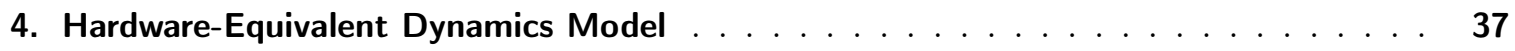

4.1. Nonlinear model . . . . . . . . . . . . . . . . . . . . . . . . 40

4.1.1. Coordinate System . . . . . . . . . . . . . . . . . . . . . . . . . . 40

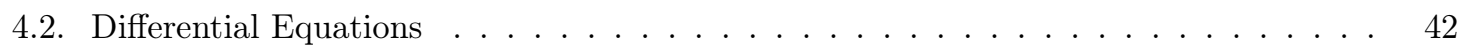

4.2.1. Wheel Angular Position $\theta$ and Body Pitch $\phi_{x} \ldots \ldots \ldots$. . . . . . 42

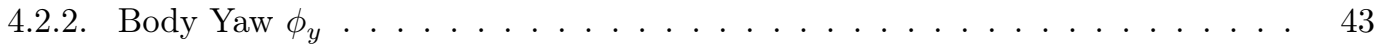

4.3. State-Space Representation . . . . . . . . . . . . . . . . . . . . 44

4.4. Calculation of Nonintuitive Parameters . . . . . . . . . . . . . . . . . 47

4.4.1. Moment of Inertia: Body: X-axis (Pitch) $J_{\phi_{x}} \ldots \ldots \ldots \ldots$. . . . . . . 47

4.4.2. Moment of Inertia: Body: Y-axis (Yaw) $J_{\phi_{y}} \ldots \ldots \ldots \ldots$. . . . . . . . 47

4.4.3. Length From Body Center of Mass to Body Axis of Rotation $l_{b . c 2 a} \ldots \ldots$

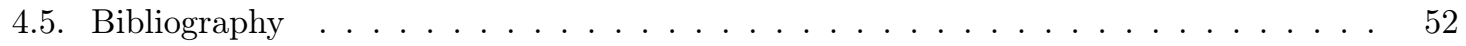

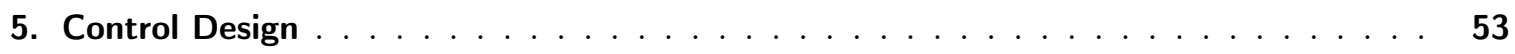

5.1. Additional Dynamics . . . . . . . . . . . . . . . . . . . . . . 53

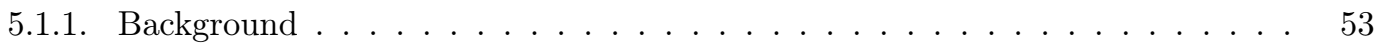

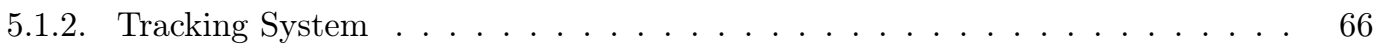

5.1.3. Control Gains . . . . . . . . . . . . . . . . . . . 68

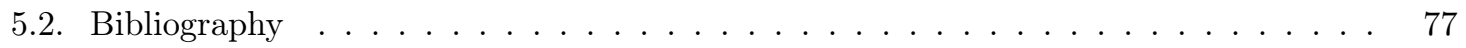

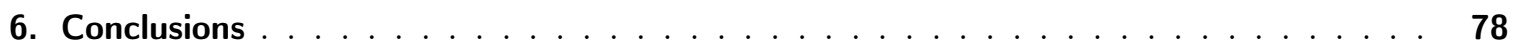

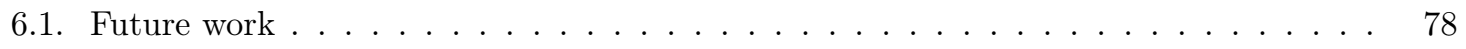

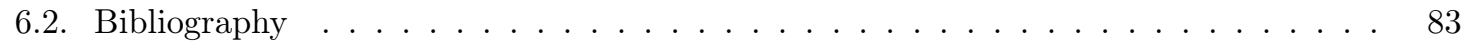

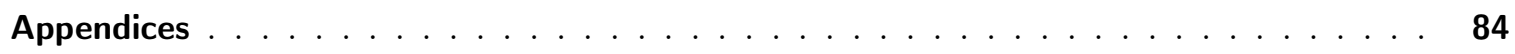

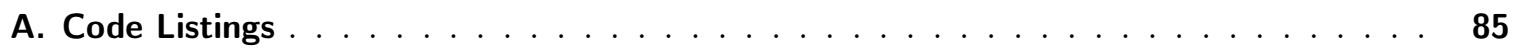

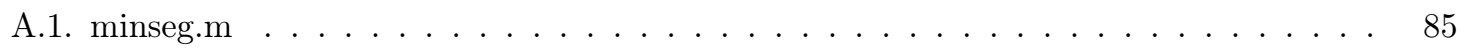

A.1.1. Global Setup . . . . . . . . . . . . . . . . . . . 88

A.1.2. User Inputs . . . . . . . . . . . . . . . . . . . . . . . . . 90

A.1.3. Initialization . . . . . . . . . . . . . . . . . 92

A.1.4. Processing . . . . . . . . . . . . . . . . . . . . . 132

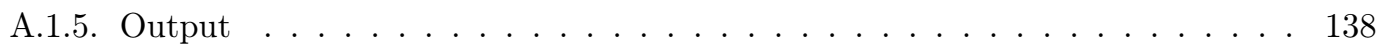

A.1.6. Global Cleanup . . . . . . . . . . . . . . . . . . . . . . . . . . . . 142 


\section{List of Figures}

2.1 [Selection of Control Problem]: Inverted Pendulum on Cart . . . . . . . . . . . 6

2.2 [Selection of Control Problem]: Two Wheeled Robot . . . . . . . . . . . . . 8

2.3 [Selection of Compatible HW \& SW]: MinSeg M2V3 (Multiview) . . . . . . . . . . 11

2.4 [Selection of Compatible HW \& SW]: MinSeg M2V3 (Exploded) . . . . . . . . . . 12

2.5 [Selection of Compatible HW \& SW]: Arduino Mega 2560 (Isometric View) . . . . . 15

2.6 [Selection of Compatible HW \& SW]: Arduino Mega 2560 (Top View) . . . . . . . 15

2.7 [Selection of Compatible HW \& SW]: Arduino Mega 2560 (Pin Map) . . . . . . . 16

2.8 [Selection of Compatible HW \& SW]: AA-Battery Voltage During Constant Discharge 17

2.9 [Selection of Compatible HW \& SW]: Motor Driver Pin Map . . . . . . . . . . . . 19

2.10 [Selection of Compatible HW \& SW]: Lego NXT Motor (3D Model) . . . . . . . . . 22

2.11 [Selection of Compatible HW \& SW]: Lego NXT Motor (Exploded) . . . . . . . . . 22

2.12 [Selection of Compatible HW \& SW]: Lego NXT Motor Gear Teeth Map . . . . . . 23

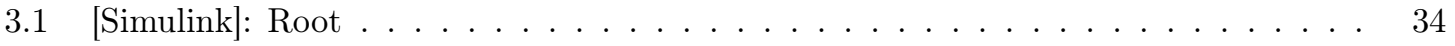

$4.1 \quad$ Hardware-Equivalent Physical Dynamics Model]: Isometric . . . . . . . . . . . . 38

4.2 [Hardware-Equivalent Physical Dynamics Model]: Multiview . . . . . . . . . . . 38

$5.1 \quad$ [Additional Dynamics]: State Feedback Regulator . . . . . . . . . . . . . . 55

$5.2 \quad$ [Additional Dynamics]: 1.0 Additional Dynamics (Design View) . . . . . . . . . . 56

5.3 [Additional Dynamics]: 1.1 Additional Dynamics (Design View) . . . . . . . . . 57

5.4 [Additional Dynamics]: 1.2 Additional Dynamics (State Feedback Regulator View) . 58

$5.5 \quad$ [Additional Dynamics]: 2.0 Additional Dynamics (Split Gains) . . . . . . . . . . 59

5.6 [Additional Dynamics]: 3.0 Additional Dynamics (Linear View: Plant) . . . . . . 60

5.7 [Additional Dynamics]: 3.1 Additional Dynamics (Linear View: Plant) _ . . . . . 61

5.8 [Additional Dynamics]: 4.0 Additional Dynamics (Linear View: Controller) . . . . . 62

5.9 [Additional Dynamics]: 4.0 Additional Dynamics with Reference Signal (Linear View:

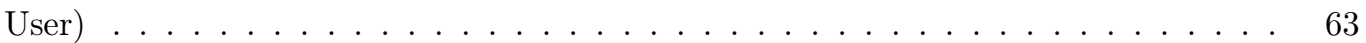

5.10 [Additional Dynamics]: 4.0 Additional Dynamics with Reference Signal (Linear View:

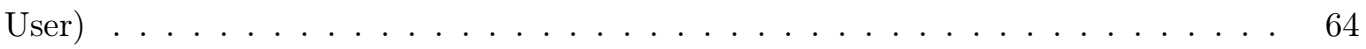

5.11 [Control Gains: LQR]: Simulation Results: Wheel Angular Position $\theta$. . . . . . . 70 
5.12 [Control Gains: LQR]: Simulation Results: Body Angular Position $\phi_{y} \ldots \ldots \ldots \ldots$. 71

5.13 [Control Gains: LQR]: Simulation Results: Body Angular Position $\phi_{x} \ldots \ldots \ldots$. . . 72

5.14 [Control Gains: LQR]: Simulation Results: Wheel Linear Position $p_{x} \ldots \ldots \ldots$

5.15 [Control Gains: LQR]: Simulation Results: Wheel Linear Position $p_{y} \ldots \ldots \ldots$. . . 74

5.16 [Control Gains: LQR]: Simulation Results: Wheel Linear Position $p_{x y} \ldots \ldots \ldots$. . 75

5.17 [Control Gains: LQR]: Simulation Results: Motor Driver Commanded Voltage $v_{\text {motor Driver }} 76$ 


\section{List of Tables}

$2.1 \quad$ Selection of Compatible HW \& SW]: MinSeg Components . . . . . . . . . . . . . 13

2.2 [Selection of Compatible HW \& SW]: Arduino Board Comparison . . . . . . . . . . 14

2.3 [Selection of Compatible HW \& SW]: Motor Driver Pin Legend . . . . . . . . . . . 19

2.4 [Selection of Compatible HW \& SW]: Motor Driver Pin Function Legend . . . . . . 20

2.5 [Selection of Compatible HW \& SW]: Motor Driver Operating Conditions . . . . . . 20

2.6 [Selection of Compatible HW \& SW]: Motor Driver Switching Characteristics . . . . 20

2.7 [Selection of Compatible HW \& SW]: Lego NXT Motor Figure Legend . . . . . . . 23

2.8 [Selection of Compatible HW \& SW]: Development PC Specifications . . . . . . . . 25

4.1 [Hardware-Equivalent Physical Dynamics Model]: Variables . . . . . . . . . . . . . . 39

4.2 [Hardware-Equivalent Physical Dynamics Model]: Parameters . . . . . . . . . . . 39 


\section{List of Code Listings}

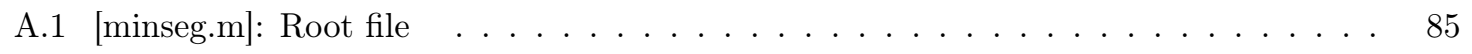

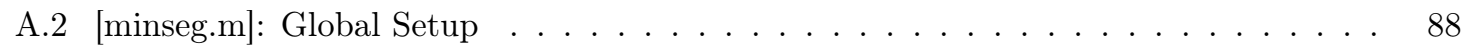

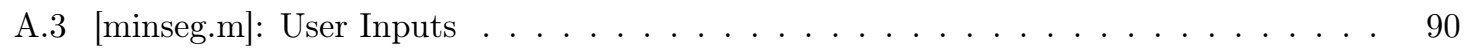

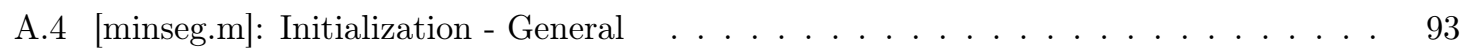

A.5 [minseg.m]: Initialization - Model - General . . . . . . . . . . . . . . . 95

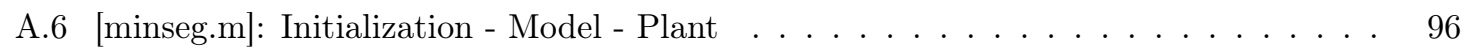

A.7 [minseg.m]: Initialization - Model - Plant - Hardware _ . . . . . . . . . . . . 98

A.8 [minseg.m]: Initialization - Model - Plant - Nonlinear Dynamics Model _ . . . . . . 103

A.9 [minseg.m]: Initialization - Model - Plant - Linear Dynamics Model _ . . . . . . . 104

A.10 [minseg.m]: Initialization - Model - Controller _ . . . . . . . . . . . . . 111

A.11 [minseg.m]: Initialization - Model - User-Defined Board Inputs and Outputs . . . 112

A.12 [minseg.m]: Initialization - Model - Model Build Parameters . . . . . . . . . . . . 114

A.13 [minseg.m]: Initialization - Serial - Write . . . . . . . . . . . . . . . . . 120

A.14 [minseg.m]: Initialization - Serial - Read . . . . . . . . . . . . . . . . 121

A.15 [minseg.m]: Initialization - Serial - General _ . . . . . . . . . . . . . . 126

A.16 [minseg.m]: Initialization - Serial - Reads . . . . . . . . . . . . . . . . . 129

A.17 [minseg.m]: Initialization - Serial - Model Build Parameters . . . . . . . . . . . . 130

A.18 [minseg.m]: Processing - Build . . . . . . . . . . . . . . . . . . . . 133

A.19 [minseg.m]: Processing - Serial - Transmit _ . . . . . . . . . . . . . . 134

A.20 [minseg.m]: Processing - Serial - Reads _ . . . . . . . . . . . . . . 136

A.21 [minseg.m]: Output - Save . . . . . . . . . . . . . . . . . . . . . . 139

A.22 [minseg.m]: Output - Serial - Reads - Plot _ . . . . . . . . . . . . . . . 140

A.23 [minseg.m]: Global Cleanup . . . . . . . . . . . . . . . . . . . . . 142 


\section{CHAPTER 1.}

\section{Introduction}

\subsection{Purpose}

The intent of this thesis is as follows:

1. Select a well-established control problem as a focal point. [Inverted Pendulum: Two-wheeled Robot]

2. Develop a modular test platform, such that differing control methods could be independently applied to the selected control problem, in real-time and in simulation.

[MinSeg Two-Wheeled Robot and Mathworks Software Suite]

3. Select or derive the dynamic equations for the physical model, and populate it. [Yamamoto [1]]

4. Select the first controller design to address the selected control problem on the test platform, and implement it in simulation.

[Optimal Controller]

5. Time permitting, implement the same controller design on the hardware. 


\subsection{Statement of the Problem}

The two-wheeled robot is a well-established control problem. The robot is topheavy and must continually work to balance itself. The robot is able to move freely on a two-dimensional plane; however, any movements performed by the robot create additional disturbances against its ability to balance itself.

Numerous command regulator approaches (PID, pole-placement, optimal) have been developed to control such a device; however, no one approach has been determined as a clear choice. Additional functionalities other than command regulators which significantly improve performance may also be implemented in a controller.

This study therefore intends to comparatively study multiple control approaches involving optimalcontrol-focused command regulators and to study the effects of additional functionalities which may be beneficial in general control cases. As a prerequisite to this work, a test platform must be developed for which to design the controllers. This study intends to design the test platform such that:

- Studies could be performed on actual hardware.

- Studies could be performed in simulation (using a hardware-equivalent model).

- Similar work involving alternate control methods could easily be incorporated.

The intent of the latter is to significantly diminish several barriers to entry to perform a control study relating to hardware (initial implementation, interfacing/communication, and theoretical/simulation modeling). This would ideally encourage future studies as well as draw them to a common platform, which would allow for effective comparisons between those studies. 


\subsection{Methodology}

The methodology of this thesis is as follows:

1. Select a well-established control problem as a focal point. [Inverted Pendulum: Two-wheeled Robot]

- Select compatible hardware and software.

$$
\left[\begin{array}{l}
\text { HW: MinSeg (Two-Wheeled Robot) } \\
\text { SW: Mathworks Matlab \& Simulink }
\end{array}\right]
$$

- Implement basic hardware-software interfaces.

- Process signals input to hardware drivers.

- Process raw signals output from hardware sensors.

$$
\left[\begin{array}{c}
\text { Datatype conversion } \\
\text { Unit conversion } \\
\text { Derivation/Integration } \\
\text { Filtration }
\end{array}\right]
$$

2. Develop a modular test platform.

- Establish infrastructure.

- Develop a unified, modular Simulink model which is capable of representing any desired system configuration.

[Variant subsystems used.]

- Create a Matlab script hierarchy which is able to:

- Configure the Simulink model to any desired system configuration.

- Configure the Simulink model to any desired build/run state.

- Organize the relatively large number of parameters involved in such a system.

- Minimize the effort required for the user to incorporate additional system configurations.

- Minimize the effort required for the user to transition between any system configurations.

- Establish robust methods of signal routing.

- Implement bus structures.

- Implement serial communication between hardware and development computer.

- Minimimize sampling interval within the limits of the board hardware.

- Process transmitted signals prior to sending and reconstruct after receiving. 
- Calibrate hardware sensors.

- Mitigate gyroscope bias.

- Develop theoretical plant model.

- Research (non-linear) physical equations.

- Linearize the physical equations.

- Develop a state-space model.

- Acquire linear plant model parameters.

- Implement linear plant model into unified test platform.

3. Design and develop and controller for the test platform.

- Implement dynamic reference tracking to mitigate bias on the body angular velocity $\phi$ sensors.

- Determine control gains using LQR. 


\subsection{Bibliography}

[1] Y. Yamamoto. (May 1, 2009). Nxtway-gs (self-balancing two-wheeled robot) controller design, [Online]. Available: https : //www . mathworks . com/matlabcentral/fileexchange/19147nxtway - gs - - self - balancing - two - wheeled - robot - controller - design (visited on 07/03/2017). 
CHAPTER 2.

\section{Preliminary Decisions}

\subsection{Selection of Control Problem}

The focal control problem was designated to be the two-wheeled robot, a special case of the inverted pendulum.

\subsubsection{Inverted Pendulum}

In control theory, the balancing of an inverted pendulum is a well-established problem [2].

In such a problem, a rigid, column-like mass is used as a pendulum. One end of the pendulum is mounted to a motoring device. The mounted end of the pendulum is granted a degree of freedom to rotate. If the pendulum is inverted (positioned in a standing position), any disturbance will ultimately tip it such that it falls.

One such system is depicted in Figure 2.1. In this simple case, the wheels of the cart allow it to move along a one-dimensional plane (a linear path).

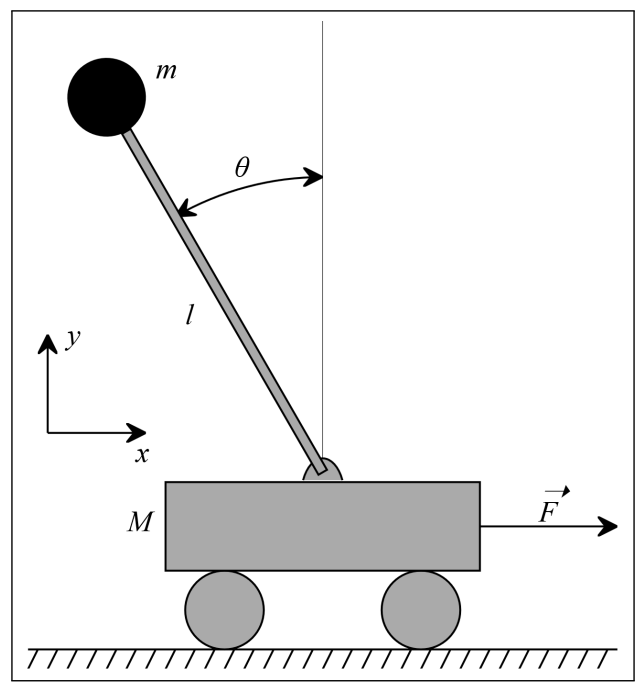

Figure 2.1.: [Selection of Control Problem]: Inverted Pendulum on Cart [3] 
The motoring device is used in such a setup to provide counterforces to the mounted end of the pendulum. These counterforces are intended to ultimately return the top of the pendulum to its inverted (standing) position.

For the actuator to successfully perform these actions, a controller (calculation device) is required. The controller, with the assistance of sensory data, is able to dynamically calculate (in real time) the exact forces needed to reestablish the positioning of the pendulum to a standing equilibrium. The controller then communicates the magnitude and direction of these forces to the motoring device which actuates the forces in the physical space. This in turn changes the state of the system, requiring that the controller continually recalculate the forces needed to return to equilibrium.

This problem may be further complicated by implementing trajectory control, in which the operator may command the device to move to one or more different locations. In such a scenario, the device must maintain its control of the balance of the inverted pendulum during and after moving.

\subsubsection{Two-Wheeled Robot}

The two-wheeled robot is a special case of the inverted pendulum model. In this case, the inverted pendulum model is reduced to only the pendulum and the wheels. The entirety of the robot hardware forms the pendulum, and the pendulum is coupled directly to the wheels.

One such device is depicted in Figure 2.2. In this case, the robot is being used in a medical application. The significance of the two-wheeled robot is not related to any one application; rather its ability to balance allows the added inclusion of top-heavy architectures in design options.

The robot has two wheels, each of which is coupled to an individual motoring device (included in the robot hardware). The motoring devices are able to act independently; therefore, the device is capable of turning and moving across a two-dimensional plane. This transition from one shaft to two shafts creates additional complexity in the system which must be considered in the design of the controller. 


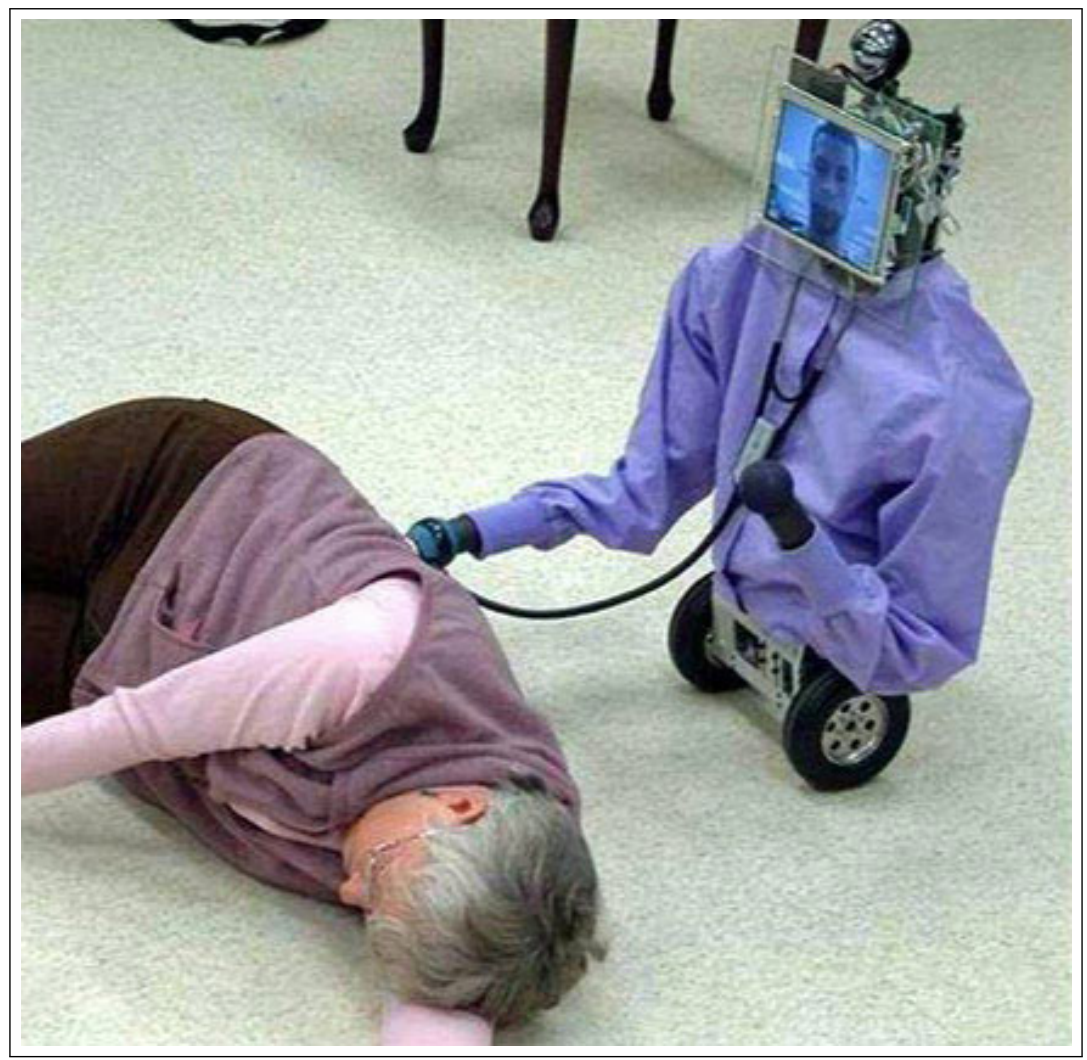

Figure 2.2.: [Selection of Control Problem]: Two Wheeled Robot [4] 


\subsection{Selection of Hardware}

The selection of hardware consists of selecting:

- A specific device to serve as the plant with respect to the designated control problem.

[MinSeg two-wheeled robot.]

- A specific device to serve as the controller with respect to the designated control problem.

[Arduino Mega2560 single-board microcontroller, included with MinSeg two-wheeled robot.]

- A specific computer to be used to interface with the controller.

[Available laptop installed with Mathworks Software Suite and supporting software.] 


\subsubsection{MinSeg M2V3 Two-Wheeled Robot}

The MinSeg two-wheeled-robot was selected as the designated hardware platform due to:

- Its standard inclusion of several components which are considered desirable with respect to performing a control study.

[See Section 2.2.1.1]

- Its existing published academic work.

[Howard and Bushnell [5]]

[This highlighted the device as a suitable hardware platform for control studies.]

- Its existing driver support [for the Mathworks software environment.].

[Mathworks [6] and Hurst [7]]

- Its use of an Arduino-brand single-board microcontroller.

[This highlighted a significant level of support for a principal component.]

- Its relatively affordable cost.

$[\sim \$ 300]$

Specifically, the MinSeg Model M2V3 [8] was selected as the designated hardware platform, due to:

- Its standard inclusion of two [equivalent but independent] motoring axes.

$\left[\begin{array}{ccc}\text { n.axes } & \text { Movement } & \\ 1 & \text { One-dimensional } & \text { (single, straight line only) } \\ 2 & \text { Two-dimensional } & \end{array}\right]$

The MinSeg M2V3 is depicted from the front, the left-side, and the rear in Figure 2.3. The MinSeg M2V3 is depicted from the front in an exploded view in Figure 2.4. In both figures, a United States quarter is depicted for the comparison of scale.

In Figure 2.4, in addition to separated motors and a separated wheel and wheel axle, two auxiliary components are depicted. To the left is a retractable USB cable used to connect to a development PC. To the right, beside the battery, are two of the same Lego component. These were used to mount and swing the robot as an uninverted pendulum, [as described in Section 4.4.3.2]. 

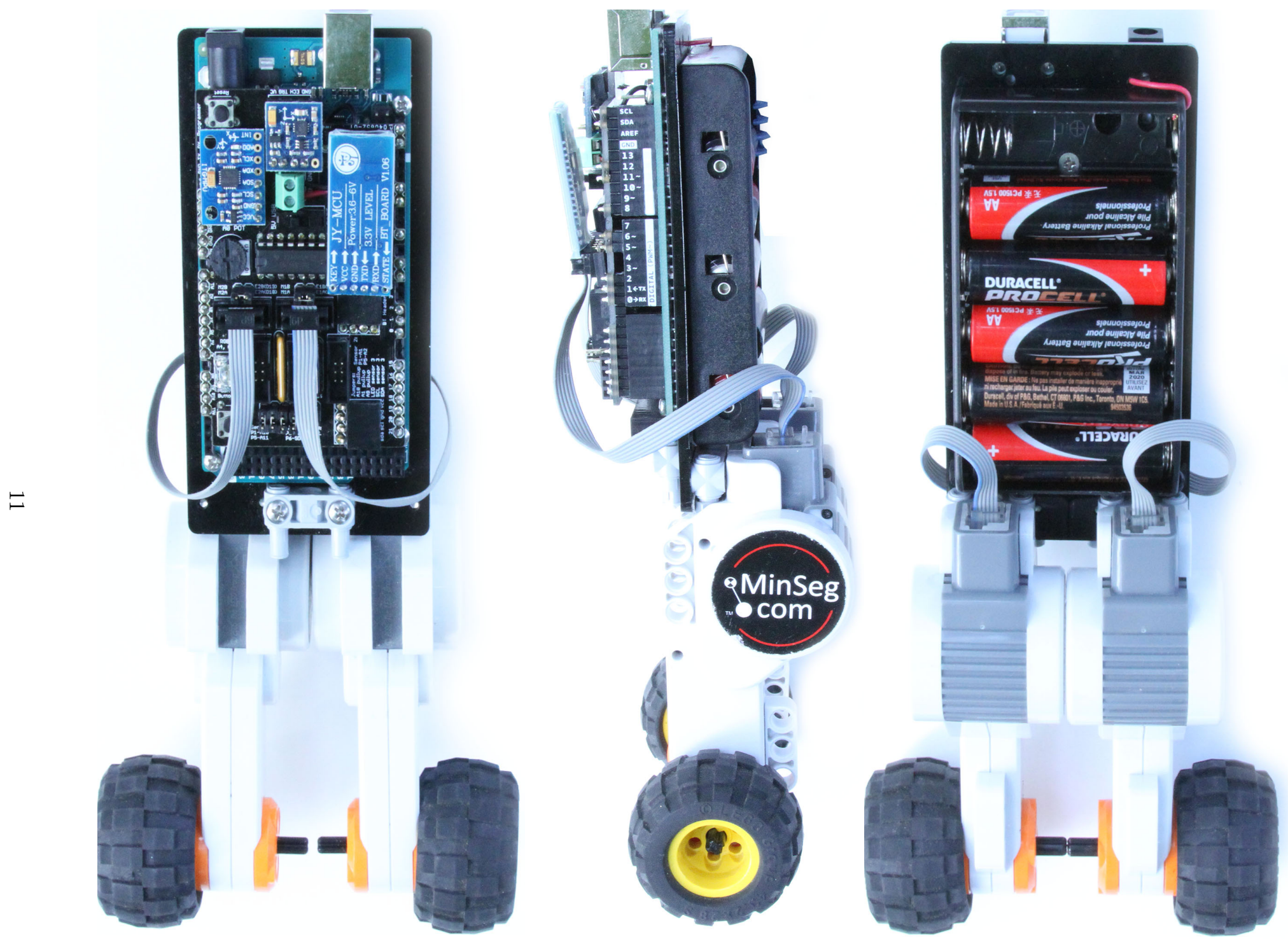

Figure 2.3.: [Selection of Compatible HW \& SW]: MinSeg M2V3 (Multiview) 


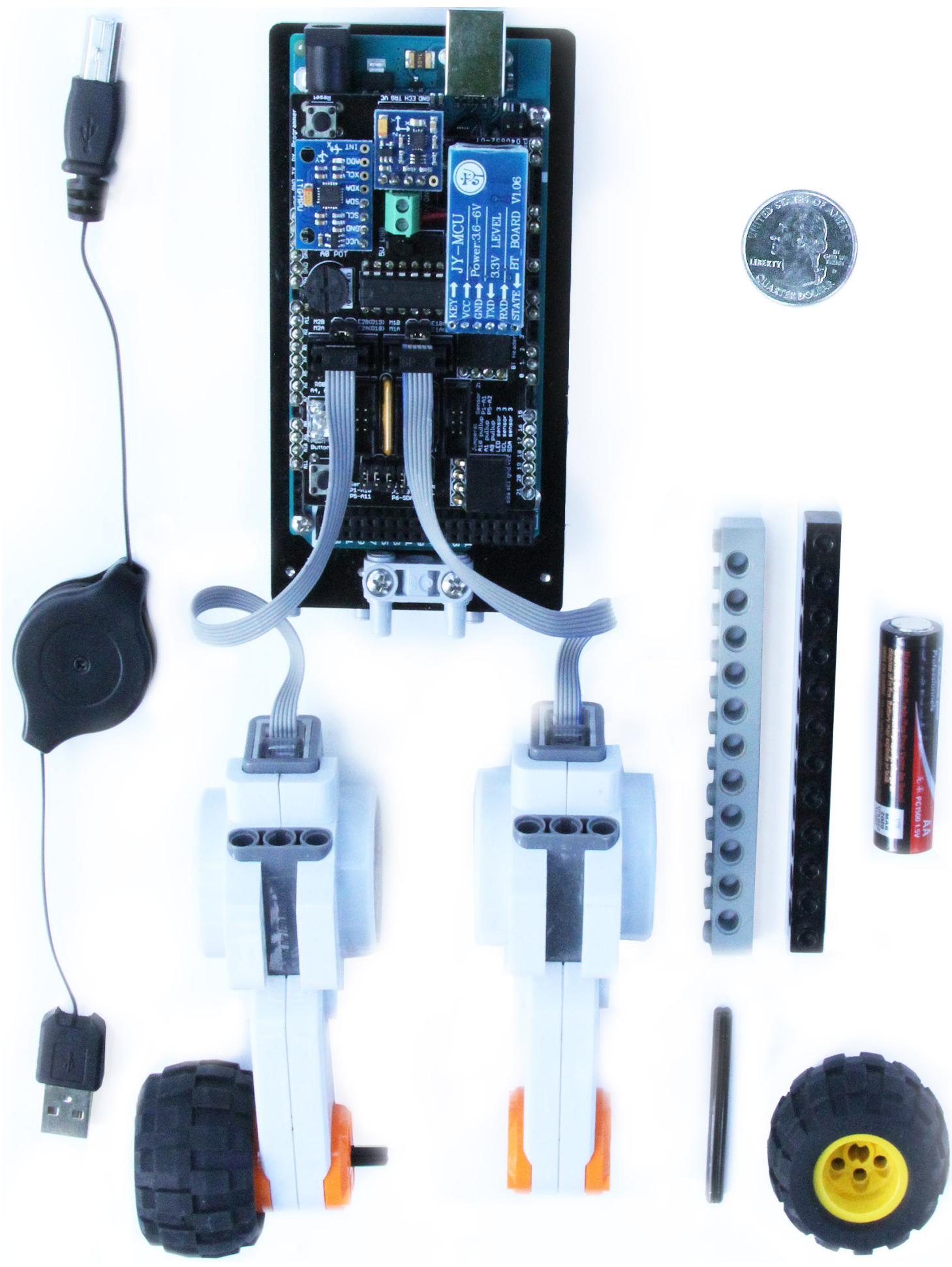

Figure 2.4.: [Selection of Compatible HW \& SW]: MinSeg M2V3 (Exploded) 


\subsubsection{Components}

As stated in Section 2.2 the MinSeg M2V3 two-wheeled robot was selected due to its inclusion of all of the desired components to perform a control study. These components are defined in Table 2.1.

Where beneficial, the components in Table 2.1 are described in greater detail in the sections which follow.

Table 2.1.: [Selection of Compatible HW \& SW]: MinSeg Components

\begin{tabular}{|c|c|}
\hline Component (Desirable) & Part Description \\
\hline Programmable microprocessor & 1x Arduino single-board microcontroller [Mega 2560] \\
\hline Dual parallel electric-driven traction motors & $\begin{array}{l}\text { 2x Lego Mindstorm NXT servo motor } \\
\qquad \text { [Includes: } 1 x \text { DC motor, } 1 x \text { gearbox, } 1 x \text { encoder }]\end{array}$ \\
\hline Tires with a relatively-high coefficient of friction & $2 \mathrm{x}$ Lego wheel $\quad[2 \mathrm{x}[43.2 \times 28]$ Balloon Small $]$ \\
\hline Motor drivers & 4x half-H-driver $\quad[1 \mathrm{x}$ SN754410] \\
\hline Sensors permitting sufficient observability of: & - \\
\hline The angular velocity of the wheels & $2 \mathrm{x}$ Encoder \\
\hline The 3-dimensional position of the body & 1x 3-axis gyroscope \& accelerometer [1x MPU6050] \\
\hline PC Communication (during operation): & - \\
\hline Wired & Serial: USB [Default. Included with microcontroller.] \\
\hline Wireless & Serial: Bluetooth $\quad$ [Supported, but sold separately. $]$ \\
\hline Power Source: & - \\
\hline Wired & $5[\mathrm{~V}]$ \\
\hline Wireless & Battery holster (6x AA) \\
\hline
\end{tabular}

\begin{tabular}{|l|lr|}
\hline \multicolumn{2}{|c|}{ Component (Unnecessary) } & \multicolumn{2}{|c|}{ Part Description } \\
\hline- & $1 \mathrm{x}$ Magnetometer & {$[1 \mathrm{x}$ HMC5883L] } \\
\hline- & $1 \mathrm{x}$ Potentiometer & {$[1 \mathrm{x} 3352]$} \\
\hline
\end{tabular}




\subsubsection{Arduino Single-Board Microcontroller}

The MinSeg M2V3 is primarily built upon an Arduino Mega 2560 single-board microcontroller. Arduino is company, project, and user-community which focuses on the development of open-source computer-hardware and software with respect to single-board microcontrollers [9]. A major boon of Arduino products is the relatively high level of support which has manifested with their popularity, including (but not limited to) the company itself, academic communities, hobbyist communities, as well as third-party private supporters such as math-software company Mathworks.

A brief comparison between the Arduino Mega 2560 and the more standard Arduino Uno is provided in Table 2.2. Most notably, the Mega 2560 has an increased number of input and output interfaces, a superior clock [10] (not apparent in the table), and increased memory versus the Arduino Uno.

Table 2.2.: [Selection of Compatible HW \& SW]: Arduino Board Comparison [11]

\begin{tabular}{|c|c|c|c|}
\hline Characteristic & Uno & Mega 2560 & Unit \\
\hline Microcontroller & ATmega328 MCU & ATmega2560 & - \\
\hline Programming Interface & $\begin{array}{c}\text { USB } \\
\text { [via ATMega16U2 ] }\end{array}$ & $\begin{array}{c}\text { USB } \\
\text { [via ATMega16U2 ] }\end{array}$ & - \\
\hline $\begin{array}{c}\text { UART } \\
\text { [Universal Asynchronous Receiver/Transmitter] }\end{array}$ & 01 & 04 & - \\
\hline Clock & ceramic resonator & crystal oscillator & - \\
\hline Clock Speed & 16 & 16 & $\mathrm{MHz}$ \\
\hline Operating Voltage & 05 & 05 & $\mathrm{~V}$ \\
\hline Number of Digital I/O [Inputs/Outputs] & 14 & 54 & - \\
\hline PWM & 06 & 14 & - \\
\hline Analog Inputs & 06 & 16 & - \\
\hline \multicolumn{4}{|l|}{ Memory/Storage: } \\
\hline Permanent (Flash) & 32 & 256 & $\mathrm{kB}$ \\
\hline Permanent $(E E P R O M)$ & 01 & 04 & $\mathrm{kB}$ \\
\hline Working (SRAM) & 02 & 08 & $\mathrm{kB}$ \\
\hline
\end{tabular}

Due to the inclusion of a USB port (which is coupled to one of the UARTs), board-to-PC interfacing (in either direction) is relatively convenient, as no special equipment is necessary (beyond a PC containing integrated development environment (IDE) software). This applies to programming the board (via the USB programming interface), as well as communicating signals during operation. 
Photos of the Arduino microcontroller are depicted in Figures 2.5 - 2.6. Additionally, a pin layout is provided in Figure 2.7.

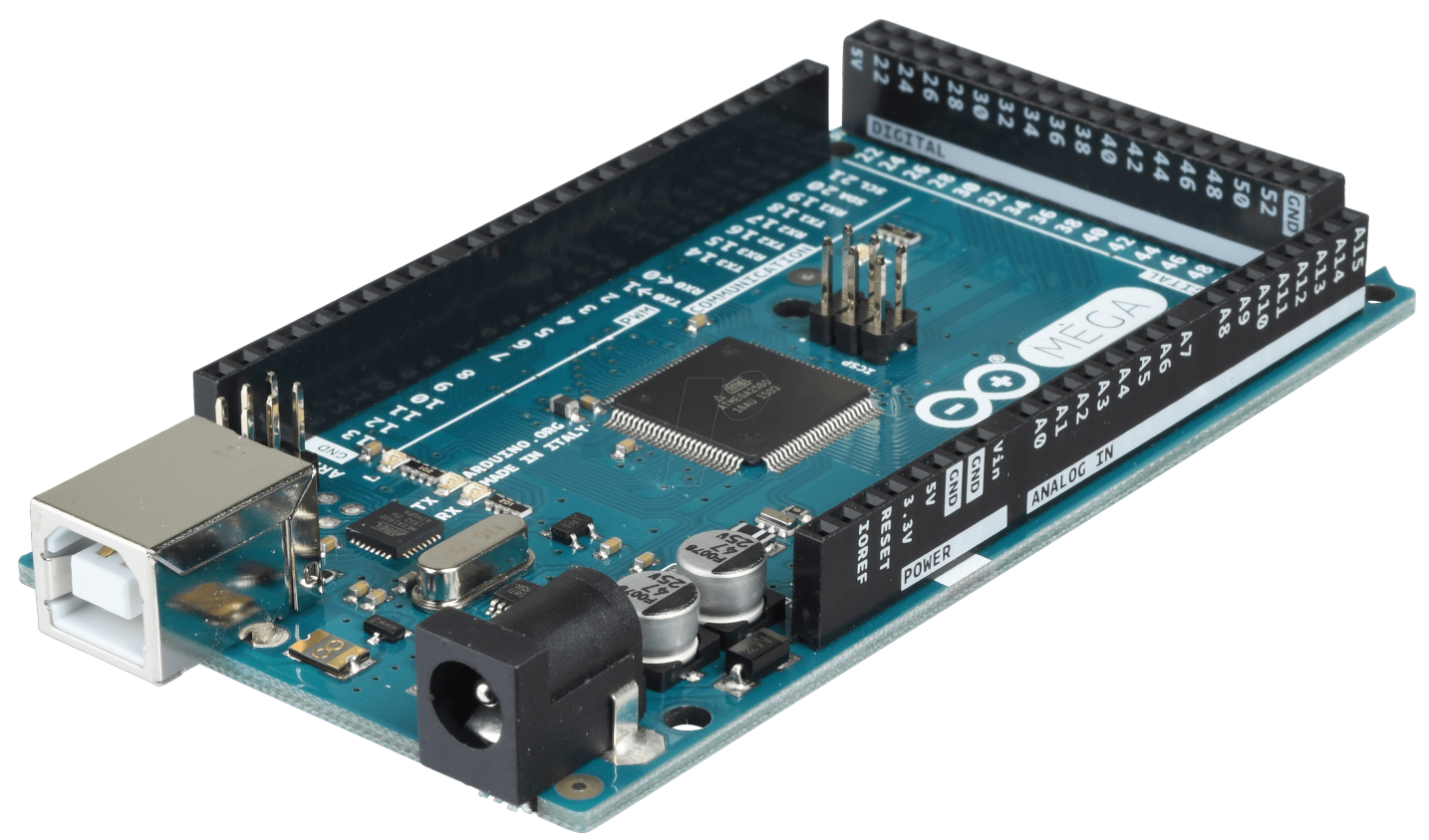

Figure 2.5.: [Selection of Compatible HW \& SW]: Arduino Mega 2560 (Isometric View) [12]

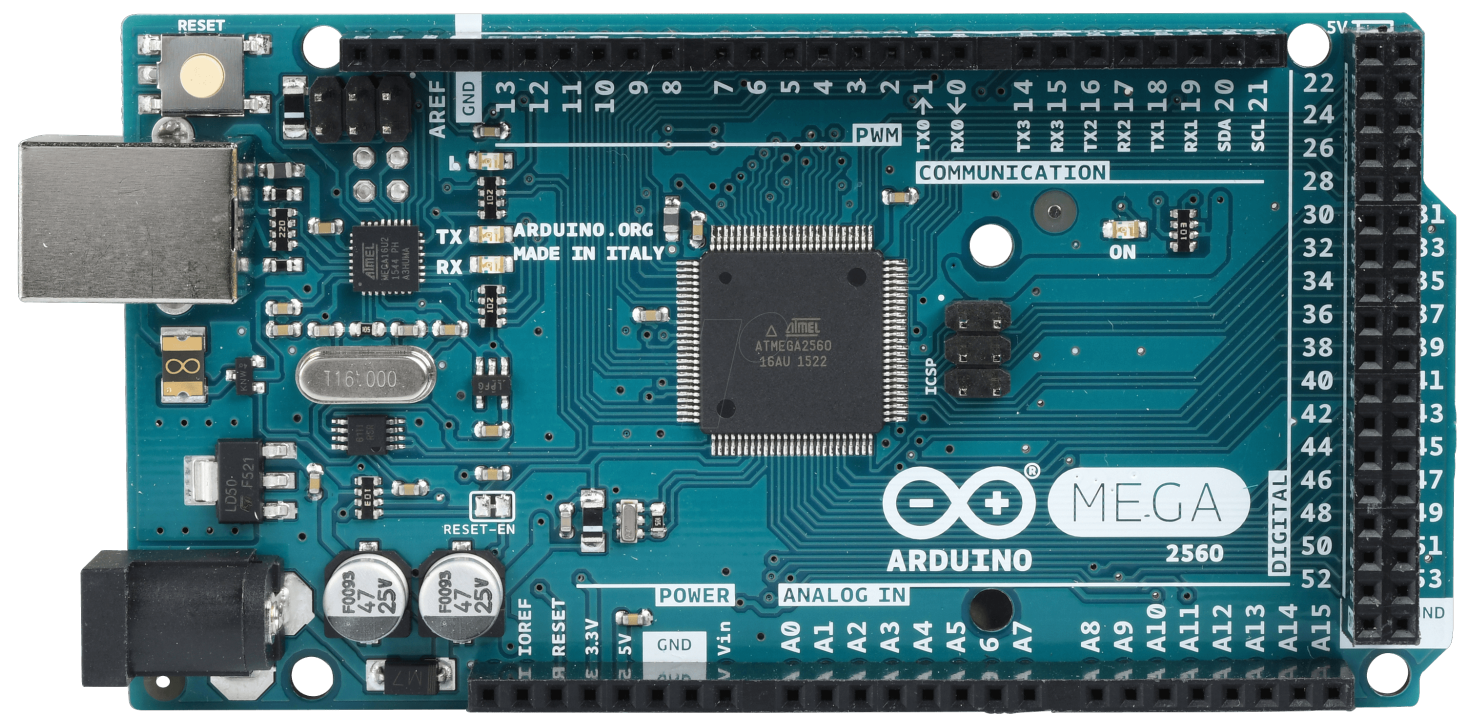

Figure 2.6.: [Selection of Compatible HW \& SW]: Arduino Mega 2560 (Top View) [12] 


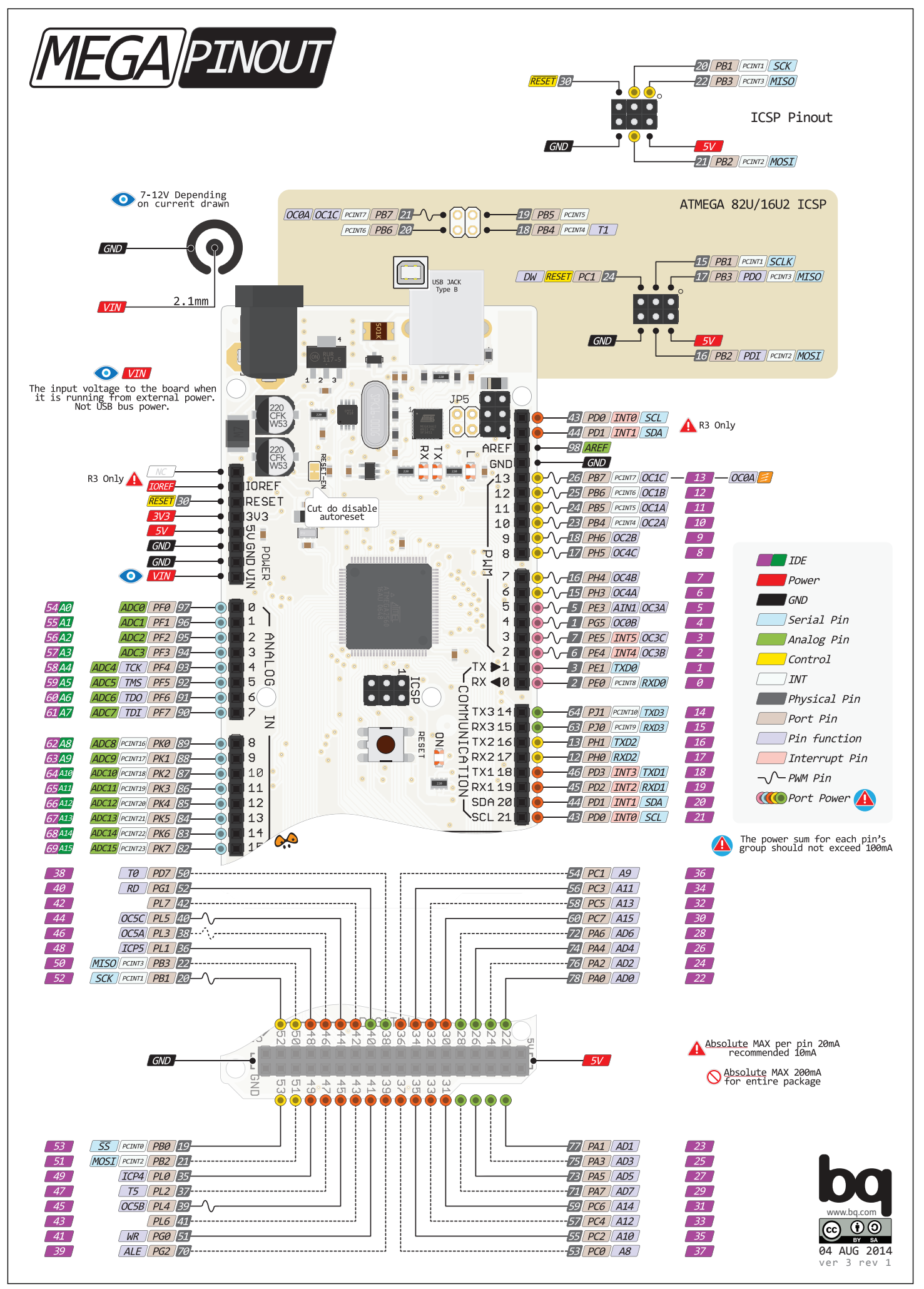

Figure 2.7.: [Selection of Compatible HW \& SW]: Arduino Mega 2560 (Pin Map) [13] 


\subsubsection{Power Source}

The MinSeg M2V3 offers two independent sources of power:

- External power via a USB port

- Internal power via an embedded battery holster

A physical switch exists on the MinSeg device to alternate between the two modes of power sourcing.

\section{External-Sourced Power (USB-Cable Connection)}

Externally-sourcing power via the USB port offers a constant 5 [V], per the USB standard; however, the cable must be consistently connected to the robot body during use.

\section{Internal-Sourced Power (Battery Pack)}

As an alternative to externally-sourced power, power may be sourced from a battery holster embedded within the MinSeg. The battery holster permits the installation of 6 AA-sized batteries.

A typical Alkaline AA-sized battery carries $1.5[\mathrm{~V}]$ at maximum charge. During use, this voltage will rapidly diminish to $\sim 1.25[\mathrm{~V}]$, and more slowly diminish from then on to $\sim 1.00[\mathrm{~V}]$ before rapidly becoming completely discharged, as depicted in Figure 2.8 .

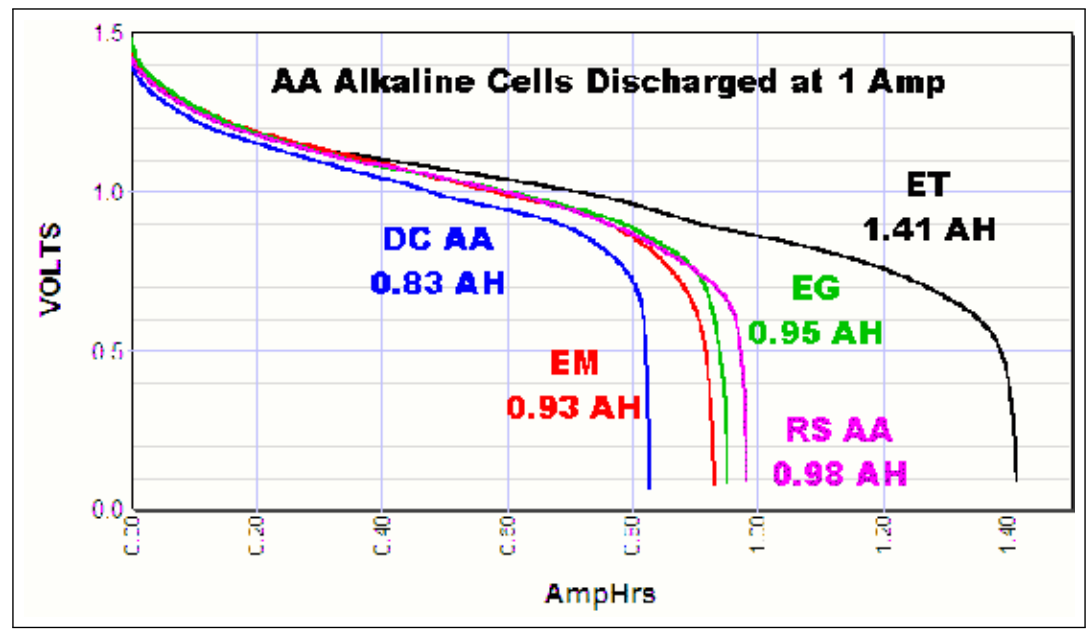

Figure 2.8.: [Selection of Compatible HW \& SW]: AA-Battery Voltage During Constant Discharge [14] 
To use the battery holster as a power source, all six AA batteries must be installed. The batteries are connected in series and therefore cumulatively offer up to $9.00[\mathrm{~V}]$ when at full charge. During typical operation, the batteries will more likely offer a reduced voltage, $\sim 7.50[\mathrm{~V}]$.

Therefore, sourcing power from the battery holster offers consistently greater voltage than external USB-connected sources, (so long as the batteries are not completely discharged), and additionally precludes the use of any wiring which could obstruct testing and operation. 


\subsubsection{Motor Driver}

The MinSeg M2V3 uses a Texas Instruments (TI) SN754410: Quadruple Half-H Driver chip as a motor driver. Supplementary information from the SN754410 datasheet is depicted in Figure 2.9 and Tables $2.3-2.6$.

Figure 2.9 and Table 2.3 exhibit that the chip has four inputs $A$ and four corresponding outputs $Y$. Table 2.5 provides a simplified description of the behavior of any one input with respect to its corresponding output:

Input $A$ acts a switch for corresponding output $Y$ :

- If the input pin $A$ is enabled $V_{I H}$, then the corresponding output $Y$ will output $V_{C C 2}[V]$.

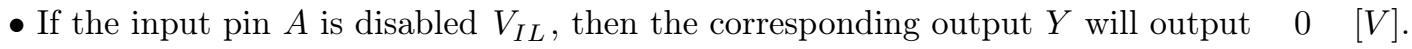

Note: It can be assumed that the enable $E N$ is engaged whenever necessary during MinSeg operation.

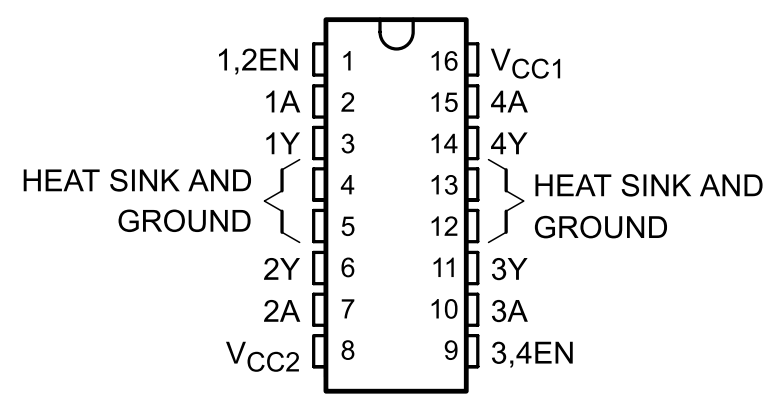

Figure 2.9.: [Selection of Compatible HW \& SW]: Motor Driver Pin Map [15]

Table 2.3.: [Selection of Compatible HW \& SW]: Motor Driver Pin Legend [15]

\begin{tabular}{|l|c|c|l|}
\hline \multicolumn{2}{|c|}{ PIN } & \multirow{2}{*}{ TYPE } & \\
\cline { 1 - 2 } NAME & NO. & I & Enable driver channels 1 and 2 (active high input) \\
\hline 1,2EN & 1 & I & Driver inputs, non-inverting \\
\hline$<1: 4>A$ & $2,7,10,15$ & O & Driver outputs \\
\hline GROUND & $4,5,12,13$ & - & $\begin{array}{l}\text { Device ground and heat sink pin. Connect to circuit board ground plane with multiple solid } \\
\text { vias }\end{array}$ \\
\hline$V_{\mathrm{CC} 2}$ & 8 & - & Power VCC for drivers 4.5V to 36V \\
\hline $3,4 \mathrm{EN}$ & 9 & $\mathrm{I}$ & Enable driver channels 3 and 4 (active high input) \\
\hline $\mathrm{V}_{\mathrm{CC} 1}$ & 16 & - & 5V supply for internal logic translation \\
\hline
\end{tabular}


Table 2.4.: [Selection of Compatible HW \& SW]: Motor Driver Pin Function Legend [15]

\begin{tabular}{|cc|c|}
\hline \multicolumn{2}{|c|}{ INPUTS (2) } & \multirow{2}{*}{ OUTPUTS } \\
\cline { 1 - 2 } A & EN & Y \\
\cline { 1 - 2 } H & H & H \\
L & $H$ & L \\
X & L & Z \\
\hline
\end{tabular}

$\mathrm{H}=$ high-level

$\mathrm{L}=$ low-level

$X=$ irrelevant

$Z=$ high-impedance (off)

Table 2.5.: [Selection of Compatible HW \& SW]: Motor Driver Operating Conditions [15]

\begin{tabular}{|c|c|c|c|c|}
\hline & & MIN & MAX & UNIT \\
\hline $\mathrm{V}_{\mathrm{CC} 1}$ & Logic supply voltage & 4.5 & 5.5 & V \\
\hline $\mathrm{V}_{\mathrm{CC} 2}$ & Output supply voltage & 4.5 & 36 & $\mathrm{~V}$ \\
\hline$V_{I H}$ & High-level input voltage & 2 & 5.5 & $\mathrm{~V}$ \\
\hline $\mathrm{V}_{\mathrm{IL}}$ & Low-level input voltage & $-0.3^{(1)}$ & 0.8 & $\mathrm{~V}$ \\
\hline $\mathrm{T}_{\mathrm{J}}$ & Operating virtual junction temperature & -40 & 125 & ${ }^{\circ} \mathrm{C}$ \\
\hline $\mathrm{T}_{\mathrm{A}}$ & Operating free-air temperature & -40 & 85 & ${ }^{\circ} \mathrm{C}$ \\
\hline
\end{tabular}

Table 2.6.: [Selection of Compatible HW \& SW]: Motor Driver Switching Characteristics [15]

\begin{tabular}{|c|c|c|c|c|c|c|}
\hline & PARAMETER & TEST CONDITIONS & MIN & TYP & MAX & UNIT \\
\hline$t_{d 1}$ & $\begin{array}{l}\text { Delay time, high-to-low-level output } \\
\text { from A input }\end{array}$ & \multirow{4}{*}{ See Figure 3} & \multicolumn{3}{|c|}{400} & ns \\
\hline$t_{d 2}$ & $\begin{array}{l}\text { Delay time, low-to-high-level output } \\
\text { from A input }\end{array}$ & & \multicolumn{3}{|c|}{800} & ns \\
\hline$t_{T L H}$ & $\begin{array}{l}\text { Transition time, low-to-high-level } \\
\text { output }\end{array}$ & & \multicolumn{3}{|c|}{300} & ns \\
\hline$t_{T H L}$ & $\begin{array}{l}\text { Transition time, high-to-low-level } \\
\text { output }\end{array}$ & & \multicolumn{3}{|c|}{300} & ns \\
\hline$t_{\text {en1 }}$ & Enable time to the high level & \multirow{4}{*}{ See Figure 4} & \multicolumn{3}{|c|}{700} & ns \\
\hline$t_{\text {en2 }}$ & Enable time to the low level & & \multicolumn{3}{|c|}{400} & ns \\
\hline$t_{\text {dis1 }}$ & Disable time from the high level & & \multicolumn{3}{|c|}{900} & ns \\
\hline$t_{\text {dis2 }}$ & Disable time from the low level & & \multicolumn{3}{|c|}{600} & ns \\
\hline
\end{tabular}


Table 2.5 specifies voltages associated with normal chip operation. The voltage source for SN754410 outputs $V_{C C 2}$ is wired to the MinSeg power source, and may therefore vary, from $4.5-9.0[\mathrm{~V}]$, (see Section 2.2.1.3).

The SN754410 inputs $A$ are connected to digital output pins on the Arduino microcontroller, specifically those which are capable of producing pulse width modulated (PWM) signals (see Sections 2.2.1.2). Programmed binary lows on the Arduino board will induce $0[V]$ and programmed binary highs will induce $5[V]$, (which is the Arduino board operating voltage).

To achieve voltages other than $V_{C C 2}$ exactly, PWM voltage signals are used. The Arduino can set its digital output pin to high for a defined fraction of the time spanning each sample interval of the Arduino board. The effect of the added switching during each sample should be considered minimal, since the MinSeg sample interval operates at the $10^{-3}[s]$ scale (as set by the operator), and the SN754410 switching interval operates at the $10^{-7}[s]$ scale, (per Table 2.6).

As stated in Section 2.2.1.1, there are two DC motors. Each has a positive and negative lead. 


\subsubsection{Motor, Gearbox, and Encoder}

The MinSeg implements two Lego NXT servo motors. Each Lego NXT servo motor contains a DC traction motor, a gearbox, and an encoder. A three-dimensional model of the component is depicted in Figure 2.10.

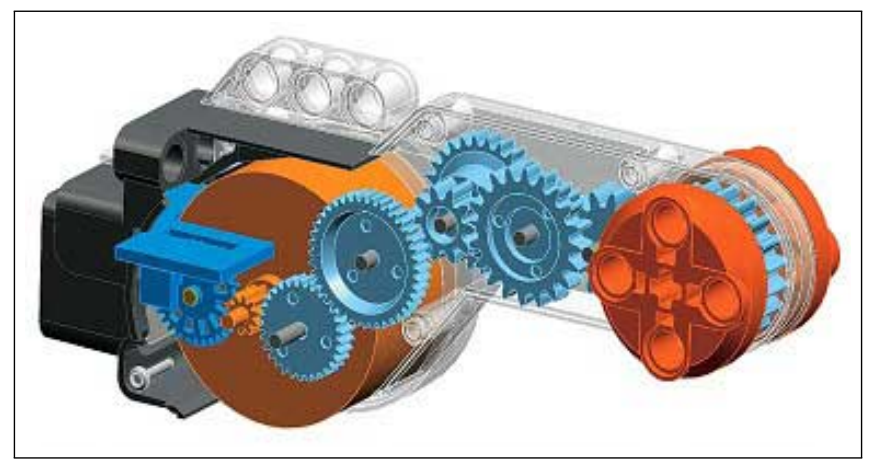

Figure 2.10.: [Selection of Compatible HW \& SW]: Lego NXT Motor (3D Model) [16]

Although Lego did not publicly disclose all of the characteristic parameters of their components, the hobbyist community reverse engineered several of these values by performing various tests and also by (irreversibly) dismantling a spare [16]. The dismantled component is depicted in Figure 2.11.

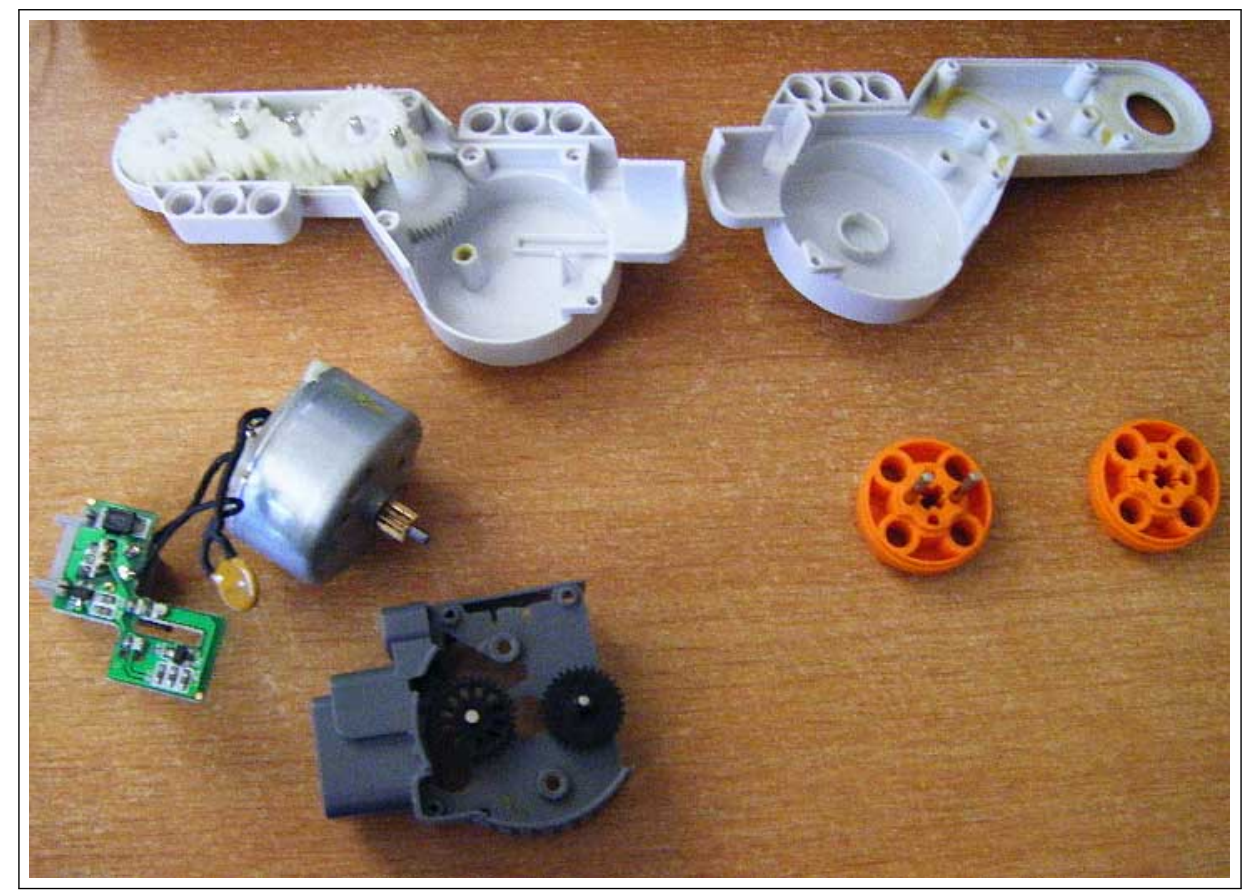

Figure 2.11.: [Selection of Compatible HW \& SW]: Lego NXT Motor (Exploded) [16] 
Table 2.7 provides a legend for the different components in Figures $2.10-2.11$.

Table 2.7.: [Selection of Compatible HW \& SW]: Lego NXT Motor Figure Legend [16]

\begin{tabular}{|l|c|c|c|}
\hline \multicolumn{1}{|c|}{ Component } & Figure 2.10 & \multicolumn{2}{c|}{ Figure 2.11 } \\
\hline Enclosure & Translucent & Top & Grey \\
\hline $\begin{array}{c}\text { Encoder } \\
\text { Enclosure } \\
\text { Gearing }\end{array}$ & - & - & - \\
Dark blue & Bottom & Black \\
PCB & Dark grey & Bottom & Dark grey \\
\hline Motor & - & Left & Green \\
\hline Gearbox & Light orange & Left & Chrome \\
\hline Wheel axle mount & Dark orange & Right & Orange \\
\hline
\end{tabular}

\section{Gearing}

The encoder, the motor, and the wheel axle mount are coupled through gearing. Thus, the angular velocity $\omega$ of any one of these components can be related to the angular velocity of any one of the other components based on the the number(s) of teeth between each component, as exhibited in Eqn. [2.1], where $k$ represents a ratio and $n$ represents an integer count.

$$
k_{\omega A 2 B}=\frac{n_{\omega B}}{n_{\omega A}}=\left[k_{\text {teeth } A 2 B}\right]^{-1}=\left[\frac{n_{\text {teeth } B}}{n_{\text {teeth } A}}\right]^{-1}
$$

The number of teeth in each gearing is depicted in Figure 2.12. Teeth counts in the same row are coupled by teeth. Teeth counts in the same column are coupled by axle, (and therefore rotate at the same rate, independent of teeth count).

$$
\begin{array}{|ccccccccccc|}
\hline 20 & \mid & 13 & 10 & \cdot & \cdot & \cdot & \mid & \cdot & \mid & \cdot \\
\cdot & \mid & \cdot & 20 & 10 & \cdot & \cdot & \mid & \cdot & \mid & \cdot \\
\cdot & \mid & \cdot & \cdot & 27 & 09 & \cdot & \mid & \cdot & \mid & \cdot \\
\cdot & \mid & \cdot & \cdot & \cdot & 40 & 30 & \mid & 10 & \mid & 32 \\
\hline
\end{array}
$$

Figure 2.12.: [Selection of Compatible HW \& SW]: Lego NXT Motor Gear Teeth Map [16] 
Additionally, in Figure 2.12, each component is separated by a vertical bar. From left to right: wheel axle mount, gearbox, motor, encoder. The completed relation between the wheel axle mount and the gearbox is exhibited in Eqns. [2.2-2.6].

$$
\begin{aligned}
k_{\text {gearTeeth wheel2motor }}= & {\left[\frac{13}{20} \cdot \frac{10}{13}\right] \cdot\left[\frac{10}{20}\right] \cdot\left[\frac{09}{27}\right] \cdot\left[\frac{30}{40} \cdot \frac{10}{30}\right]=\frac{01}{48} } \\
k_{\text {gearTeeth motor2encoder }}= & {\left[k_{\text {gearTeeth wheel2motor }}\right]^{-1}=\frac{48}{01} } \\
k_{\omega \text { wheel2motor }}= & {\left[k_{\text {gearTeeth motor2encoder }}\right]=\frac{32}{10} } \\
k_{\omega \text { motor2encoder }}= & k_{\omega \text { wheel2motor }} \cdot k_{\omega} \text { motor2encoder }=\frac{15}{01} \\
k_{\omega \text { wheel2encoder }}= &
\end{aligned}
$$




\subsubsection{Development PC}

Designations pertaining to the development $\mathrm{PC}$ with respect to the test platform are exhibited in Table 2.8.

Table 2.8.: [Selection of Compatible HW \& SW]: Development PC Specifications [14]

\begin{tabular}{|l|c|}
\hline Hardware & Version \\
\hline PC & 2015 Macbook Pro [17] \\
\hline
\end{tabular}

\begin{tabular}{|l|c|}
\hline Software & Version \\
\hline Operating System (OS) & macOS 10.12.5 \\
\hline Mathworks Software Suite & - \\
$\cdot$ MATLAB & - \\
- Simulink & - \\
- Control System Toolbox & - \\
- DSP System Toolbox & - \\
- Instrument Control Toolbox & - \\
- MATLAB Coder & - \\
- Simulink Coder & - \\
- Simulink Desktop Real-Time & 17.1 .0 \\
- Matlab Support Package for Arduino Hardware & 17.1 .0 \\
- Simulink Support Package for Arduino Hardware & 7.3 .1 \\
\hline Xcode $\quad[$ A Mathworks (macOS)-Supported Compiler [18].] & 1.1 \\
\hline Rensselaer Arduino Support Package Library (RASPLib) & \\
\hline [A third-party Simulink Support Package for MinSeg Hardware [7]].] & \\
\hline
\end{tabular}




\subsubsection{Designated PC}

A 2015 Macbook Pro PC was selected as the designated development PC, as this was available to the researcher without the need to request additional funding.

\subsubsection{Designated Operating System}

macOS was selected as the designated operating system, as this was the only operating system installed on the designated PC. (Version 10.12.5 was the most up to date version at the time of research.)

\section{Alternative Operating System Compatibility}

Although the macOS operating system was used, alternative operating systems (Windows and/or Linux) would be equally acceptable.

Such a transition would primarily require an alternative Mathworks-supported compiler [18] which would be compatible with the new operating system. Slight alterations to the method of determining the test platform serial communication channelwould also be required.

It is not expected that such a transition would be preventatively difficult. 


\subsubsection{Designated Hardware-Interfacing Software}

The Mathworks Software Suite was selected as the designated hardware-interfacing software due to:

- Its first-party support for programming real-time hardware.

- Its first-party support for simulating real-time hardware.

- Its first-party driver support for Arduino-brand microcontrollers.

- Its third-party driver support for the MinSeg.

- Its first-party support for serial communication with hardware in real-time.

- Its relatively user-friendly language and interfaces.

- Its relative commonality among students and academic institutions.

$\left[\begin{array}{r}\text { The software environment was already relatively familiar to } \\ \text { the author and to the advising professor prior to performing this study. }\end{array}\right]$

- Its relatively affordable cost.

[With respect to students and academic institutions. $\sim$ \$150]. 


\subsection{Selection of a Hardware Model}

The physical plant model developed in Yamamoto [1] was used, due to:

- Use of state variables involving:

- Body pitch angle $\alpha$

- Body yaw angle $\Psi$

- Wheel angle $\theta$

- Existing familiarity of the work by the advising professor.

- Existing knowledge of methods to measure nonintuitive model parameters by the advising professor.

The physical plant model is discussed in greater detail in Section ??. 


\subsection{Selection of Controller Design}

Since pole-placement methods had been researched relatively recently under the advising professor, optimal control techniques were researched.

This is discussed in greater detail in Section 5 . 


\subsection{Bibliography}

[1] Y. Yamamoto. (May 1, 2009). Nxtway-gs (self-balancing two-wheeled robot) controller design, [Online]. Available: https : //www . mathworks . com/matlabcentral/fileexchange/19147nxtway - gs - - self - balancing - two - wheeled - robot - controller - design (visited on $07 / 03 / 2017)$.

[2] R. J. Vaccaro, Digital Control: A State-space Approach, ser. Mcgraw Hill Series in Electrical and Computer Engineering. Mcgraw-Hill College, Jan. 1995, ISBN: 978-0070667815.

[3] Wikipedia. (Jun. 24, 2017). Inverted pendulum, Wikimedia Foundation, [Online]. Available: https://en.wikipedia.org/wiki/Inverted_pendulum (visited on 07/03/2017).

[4] D. Melanson. (Jul. 14, 2016). Researchers create life-saving ubot-5 robot, play dress-up with it, Engadget, [Online]. Available: https ://www . engadget.com/2008/04/17/researcherscreate-life-saving-ubot-5-robot-play-dress-up-with/ (visited on 02/05/2017).

[5] B. Howard and L. Bushnell, "Enhancing linear system theory curriculum with an inverted pendulum robot," in 2015 International Conference on Computer Science and Mechanical Automation (CSMA), IEEE, Hangzhou, China, Oct. 2015. DOI: 10.1109/CSMA .2015.63.

[6] Mathworks. Arduino programming with matlab and simulink, [Online]. Available: https: //www . mathworks . com/discovery/arduino-programming-matlab-simulink.html (visited on $07 / 03 / 2017)$.

[7] J. Hurst. (Jun. 17, 2016). Rensselaer arduino support package library (rasplib), 1.1, Rensselaer Polytechnic Institute (RPI), [Online]. Available: https : / / www . mathworks . com / matlabcentral/fileexchange/62702-rensselaer-arduino-support-package-library-rasplib- (visited on $07 / 03 / 2017$ ).

[8] MinSeg. M2v3, [Online]. Available: https : //minseg . com/collections/minseg-kits / products/minsegshield-kit-m2v3-2-dual-drive-segway-and-line-follower (visited on $07 / 03 / 2017)$.

[9] Wikipedia. (Jul. 1, 2017). Inverted pendulum, Wikimedia Foundation, [Online]. Available: https://en.wikipedia.org/wiki/Arduino (visited on 07/03/2017).

[10] jkenny23. (May 9, 2011). Crystal vs. resonator, Arduino, [Online]. Available: https://forum. arduino.cc/index.php?topic=60662.0 (visited on 07/03/2017). 
[11] Arduino Geeks. (Feb. 2, 2017). Arduino mega vs uno compared, Arduino Starter Kits, [Online]. Available: https : / / www . arduinostarterkits . com / reviews / arduino-mega - vs - uno compared/ (visited on 07/03/2017).

[12] Reichelt Elektronik. Arduino mega: Arduino mega 2560, atmega1280, usb, [Online]. Available: https : //www . reichelt. com/de/en/Single-board-microcontroller/ARDUINO-MEGA/3/ index $\cdot h t m l$ ?ACTION=3\&GROUPID=6667\&ARTICLE=119696 (visited on 07/03/2017).

[13] Alberto "PighiXXX". (Aug. 4, 2014). Mega, PighiXXX, [Online]. Available: http: //www . pighixxx.com/test/portfolio-items/mega/ (visited on 07/03/2017).

[14] PowerStream. (Jun. 29, 2017). Discharge tests of aa batteries, alkaline and nimh, [Online]. Available: https://www. powerstream.com/AA-tests.htm (visited on 07/03/2017).

[15] Texas Instruments. (Jan. 2015). Sn754410: Quadruple h drivers, [Online]. Available: http: //www.ti.com/product/sn754410?qgpn=sn754410 (visited on 07/03/2017).

[16] P. "Philo" Hurbain. (May 15, 2017). Nxt motor internals, [Online]. Available: http://www . philohome.com/nxtmotor/nxtmotor.htm (visited on 07/03/2017).

[17] Everymac. (May 22, 2017). Apple macbook pro "core i5" 2.7 13" early 2015 specs, [Online]. Available: http://www. everymac.com/systems/apple/macbook_pro/specs/macbook-procore-i5-2.7-13-early-2015-retina-display-specs.html (visited on 07/03/2017).

[18] Mathworks. Previous releases: System requirements and supported compilers, [Online]. Available: https://www . mathworks.com/support/sysreq/previous_releases.html (visited on $07 / 03 / 2017)$. 


\section{CHAPTER 3.}

\section{Test Platform}

The test platform consists of the designated hardware, [MinSeg M2V3 two-wheeled robot, see Section 2.2.1], and the designated development PC, [see Section 2.2.2]. To interface with the hardware, a Simulink model and a hierarchy of Matlab subscripts were created.

The Simulink model is capable of:

- Acting as an algorithm with which to program the hardware, such that it may:

- Process

- Actuate

- Communicate

- Simulate an equivalent model of "the hardware when loaded with the same algorithm".

The Matlab script hierarchy is capable of:

- Initialize model parameters.

- Reconfigure model subsystems.

- Initialize a build or simulate event.

- Initialize a read or write event.

- Post-process raw read data.

- Save processed read data as well as other configuration data.

- Plot processed read data. 


\subsection{Simulink: minseg_M2V3_2017a.slx}

minseg_M2V3_2017a.slx is the label of the Simulink model file. The label includes the label of the hardware which it represents as well as the version of the Mathworks Software Suite with which it

was created. [Using the model in a different version of the Simulink will require conversion; therefore, the two files will not be equivalent.]

The Simulink model is hierarchical. The sections which follow will describe the model and will be similarly organized, as depicted in the extended-precision List of Contents below.

\subsubsection{Root}

The top level of the model, also known as the model root, is depicted in Figure 3.1.

The model root is contains the three primary components of the system:

- Plant

- Controller

- Board Inputs and Outputs 


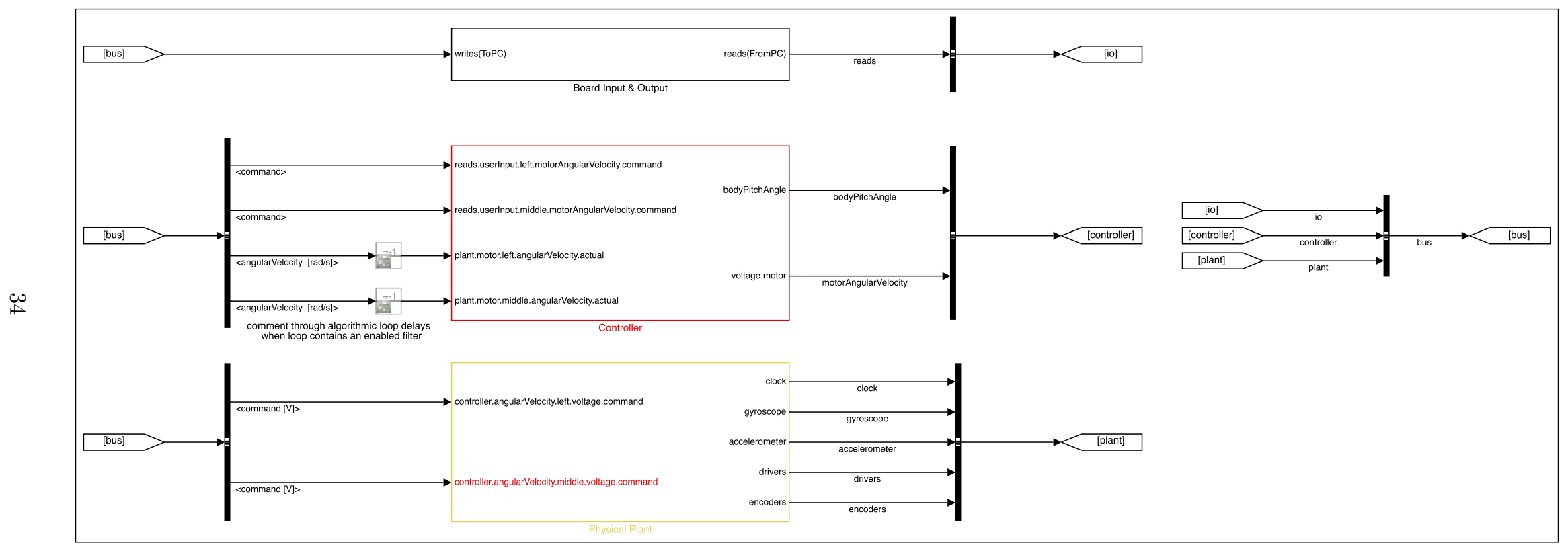

Figure 3.1.: [Simulink]: Root 


\subsubsection{Bus Structures}

Bus structures are a means of routing large quantities of signals. They are similar to muxed signals; however, it is not necessary to separate all of the signals during the demux process.

It is evident in Figure 3.1 that all of the components are passed into separate bus structures, [black bars on the right-side of the figure], and that those bus structures are in turn merged into one global bus structure.

This grants the user the ability to call any significant signal wherever it is needed using bus selectors, [black bars on the left-side of the figure]. The user should take care to implement a delay in the path of any signal which is implemented recursively [as feedback]. [This prevents the formation of an algebraic loop].

\subsubsection{Variant Subsystems}

A variant subsystem is a subsystem containing multiple subsystems, defined as variants. Only one variant can be active at one time. The variant subsystem serves as the switch between them. [Note that the variant subsystem cannot switch between variants during operation/runtime].

Several subsystems contained in this model are variant subsystems. These variant subsystems are used to switch system configurations. Examples of these variant configurations include:

- The plant:

- Actual hardware drivers.

[Hardware implementation only.]

- Hardware-equivalent simulation model of nonlinear dynamics.

[Simulation only.]

- Hardware-equivalent simulation model of linear dynamics.

[Simulation only.]

- The controller design:

- PID.

[Primarily for initial hardware characterization.]

- Optimal.

- Pole-placement. 


\subsection{Matlab: minseg.m}

The minseg.m script was developed to control the MinSeg test platform. The script is capable of:

- Reconfiguring the model

- Running a model simulation

- Programming the model hardware

- Communicating with the model hardware

- Optimizing the communication rate.

- Reformatting the raw hardware-output data on receipt.

- Saving the initialization parameters and output data.

- Plotting the output data.

The script is hierarchal, and is therefore only the root or master file to a series of subfiles. The subfiles are broken up into principal segments of the scripting process:

- Global setup

- User-input

- Initialization

- Processing

- Output

- Global Cleanup 


\section{CHAPTER 4.}

\section{Hardware-Equivalent Dynamics Model}

To simulate the dynamics of the hardware, a hardware-equivalent dynamics model was selected.

The model was originally derived by Yamamoto [1] and has been successfully used in other control studies [19].

Figures $4.1-4.2$, depict the physical model of the two-wheeled inverted pendulum as isometric and multiview projections. These figures use Yamamoto's original symbol notation; a legend is provided in Figure 4.2.

Yamamoto [1] makes the following assumptions in Figures 4.1 - 4.2:

- All mass geometries are uniform.

- All masses are uniformly distributed.

- The hardware consists of three principal masses:

- A rectangular cuboid

[The body.]

- A cylinder

[The left wheel.]

- A cylinder

[The right wheel.]

Tables $4.1-4.2$, define the variables and the parameters, respectively, that the physical model of the two-wheeled inverted pendulum will use. 


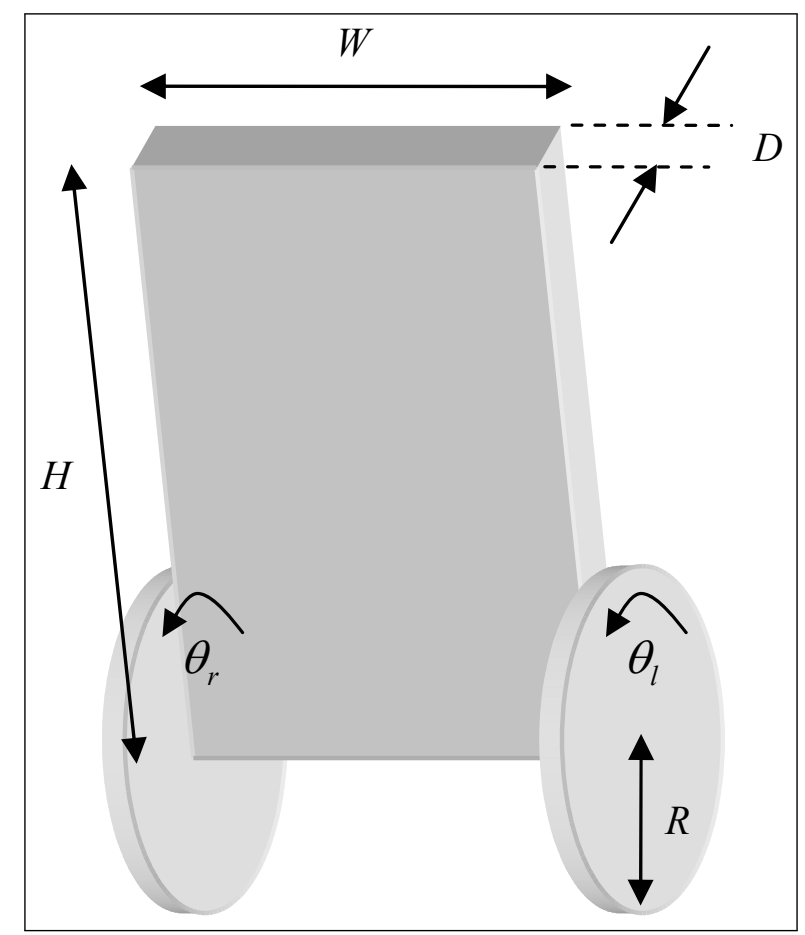

Figure 4.1.: [Hardware-Equivalent Physical Dynamics Model]: Isometric[1]

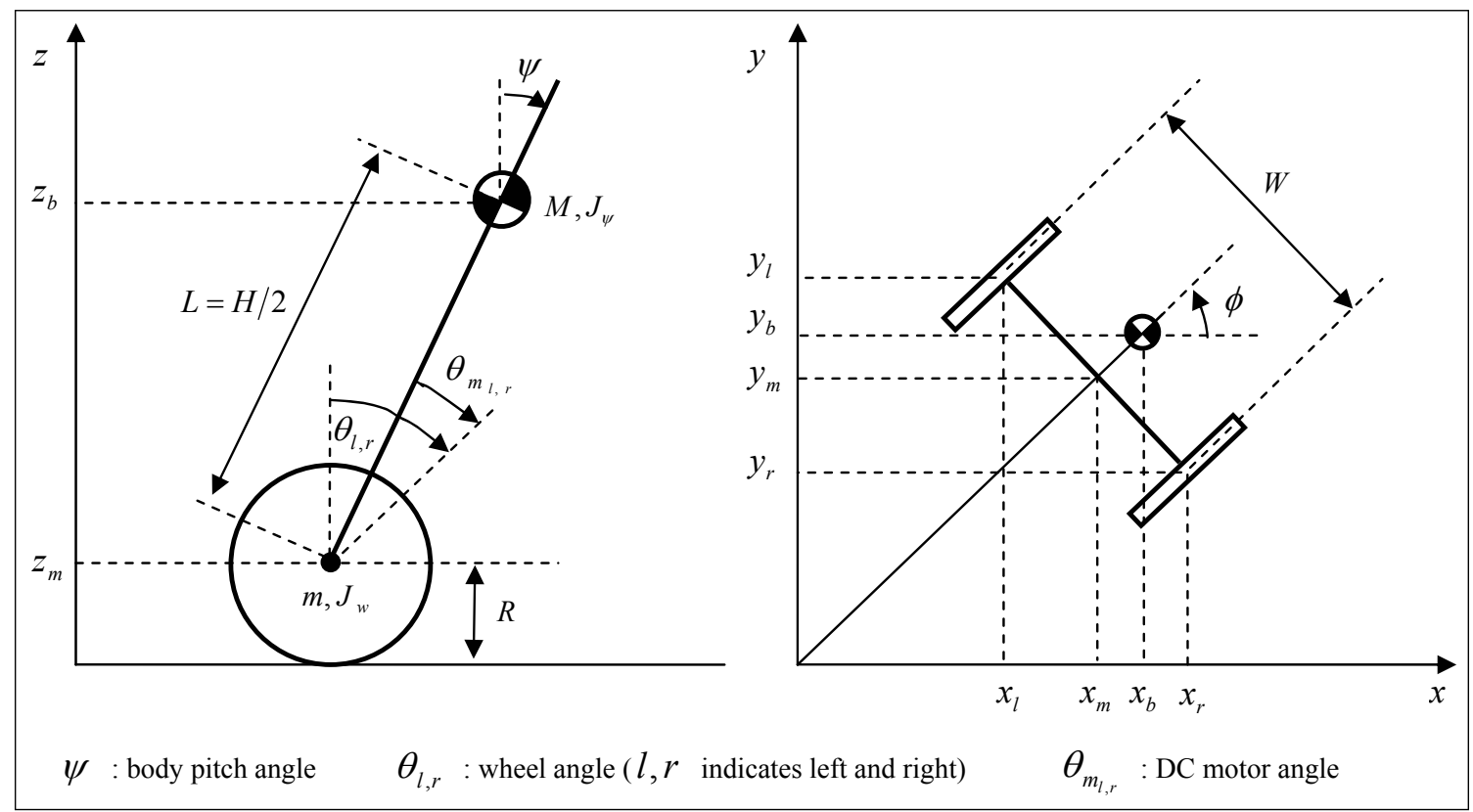

Figure 4.2.: [Hardware-Equivalent Physical Dynamics Model]: Multiview[1] 
Table 4.1.: [Simulink]: Root

\begin{tabular}{|c|c|c|c|}
\hline Symbol & & Definition & Unit \\
\hline$\theta$ & Angular position: Wheel & {$\left[\theta=\theta_{g, a v}\right][$ Measured from the wheel center of mass] } & \\
\hline$\theta_{g}, \theta_{b}$ & Origin aligns with: & {$\left[\begin{array}{c}\text { global rejection vector } \\
\text { (orthogonal from earth's surface) }\end{array}\right]\left[\right.$ body pitch $\left.\phi_{x}\right]$} & $\mathrm{rad}$ \\
\hline$\theta_{a v}, \theta_{l}, \theta_{r}$ & Component: & [ average of left and right wheels ] [ left wheel ] [ right wheel ] & \\
\hline$\phi$ & Angular position: Body & [Measured from the wheels center of mass.] & rad \\
\hline$\phi_{x}, \phi_{y}, \phi_{z}$ & Dimension: & {$[\mathrm{X}][\mathrm{Y}][\mathrm{Z}]$} & \\
\hline$p$ & Translational position & {$\left[p=p_{w}\right]$ [Measured from the corresponding center of mass] } & \\
\hline$p_{x}, p_{y}, p_{z}$ & Dimension: & {$[\mathrm{X}] \quad[\mathrm{Y}][\mathrm{Z}]$} & $m$ \\
\hline$p_{w}, p_{w l}, p_{w r}, p_{b}$ & Component: & [ both wheels ] [ left wheel ] [ right wheel ] [ body ] & \\
\hline$v_{m t r}$ & Voltage: Motor Input & & $V$ \\
\hline$v_{m t r . l}, v_{m t r . r}$ & Component: & [ left-wheel ] [ right-wheel ] & \\
\hline
\end{tabular}

Note: When a subscript is unspecified, assume the first option is used by default.

Table 4.2.: [Simulink]: Root

\begin{tabular}{|c|l|c|c|c|}
\hline Symbol & \multicolumn{1}{|c|}{ Definition } & Value & Unit & Source \\
\hline$a_{g}$ & Acceleration of gravity: Earth & 9.81 & $\frac{m}{s^{2}}$ & - \\
\hline$m_{w}$ & Mass: Wheel $\quad$ [Includes wheel axle.] & 0.018 & $\mathrm{~kg}$ & {$[5]$} \\
\hline$m_{b}$ & Mass: Body & 0.381 & $\mathrm{~kg}$ & {$[5]$} \\
\hline$l_{b . h}$ & Length: Body: Height & $? ?$ & $m$ & - \\
\hline$l_{b . w}$ & Length: Body: Width & $? ?$ & $m$ & - \\
\hline$l_{b . d}$ & Length: Body: Depth & $? ?$ & $m$ & - \\
\hline$l_{b . c 2 a}$ & Length: Body: [Center of mass] to [Axis of Rotation] & $? ?$ & $m$ & Sec. 4.4 .3 \\
\hline$r_{w}$ & Length: Wheel: Radius & 0.021 & $m$ & {$[5]$} \\
\hline$J_{b . \phi_{x}}$ & Moment of Inertia: Wheel & $7.46 \cdot 10^{-6}$ & $\mathrm{~kg} \cdot \mathrm{m}^{2}$ & {$[5]$} \\
\hline$J_{b . \phi_{x}}$ & Moment of Inertia: Body: X-axis (pitch) & $? ?$ & $\mathrm{~kg} \cdot \mathrm{m}^{2}$ & Sec. 4.4 .1 \\
\hline$J_{b . \phi_{y}}$ & Moment of Inertia: Body: Y-axis (yaw) & $? ?$ & $\mathrm{~kg} \cdot \mathrm{m}^{2}$ & Sec. 4.4 .2 \\
\hline$R_{m t r}$ & Motor: Resistance & 4.4 & $\Omega$ & {$[5]$} \\
\hline$k_{m t r . b E M F}$ & Motor: Coefficient of Back EMF & 0.495 & $\frac{V \cdot s}{r a d}$ & {$[5]$} \\
\hline$k_{m t r . T}$ & Motor: Coefficient of Torque & 0.470 & $\frac{N \cdot m}{A}$ & {$[5]$} \\
\hline$k_{f r . m 2 w}$ & Motor: Coefficient of friction: [DC Motor] to [Wheel] & $? ?$ & - & Sec. ?? \\
\hline
\end{tabular}




\subsection{Nonlinear model}

The dynamic motion equations of the two-wheeled robot are derived using the Lagrangian method. The equations are based on the coordinate system provided in Figure 4.2.

\subsubsection{Coordinate System}

The coordinate system is explicitly defined in Equations (4.1) - (4.6).

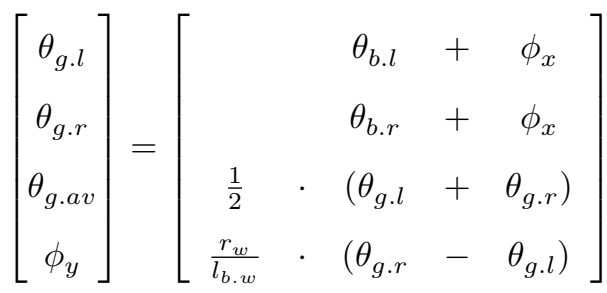

$\left[\begin{array}{c}\dot{p}_{w \cdot x} \\ \dot{p}_{w \cdot y} \\ \dot{p}_{w . z}\end{array}\right]=\left[\begin{array}{c}r_{w} \cdot \dot{\theta}_{g . a v} \cdot \cos \left(\phi_{y}\right) \\ r_{w} \cdot \dot{\theta}_{g \cdot a v} \cdot \sin \left(\phi_{y}\right) \\ 0\end{array}\right]$

$\left[\begin{array}{c}p_{w \cdot x} \\ p_{w \cdot y} \\ p_{w \cdot z}\end{array}\right]=\left[\begin{array}{ccc}\int \dot{p}_{w . x} \cdot \mathrm{d} t+ & p_{w \cdot x}(0) \\ \int \dot{p}_{w \cdot y} \cdot \mathrm{d} t+ & p_{w \cdot y}(0) \\ \int \dot{p}_{w . z} \cdot \mathrm{d} t+ & p_{w . z}(0)\end{array}\right]$

$\left[\begin{array}{l}p_{w l . x} \\ p_{w l . y} \\ p_{w l . z}\end{array}\right]=\left[\begin{array}{cc}p_{w . x} & -\frac{l_{b . w}}{2} \cdot \sin \left(\phi_{y}\right) \\ p_{w . x} & +\frac{l_{b . w}}{2} \cdot \cos \left(\phi_{y}\right) \\ & p_{w . z}\end{array}\right]$

$\left[\begin{array}{l}p_{w r . x} \\ p_{w r . y} \\ p_{w r . z}\end{array}\right]=\left[\begin{array}{c}p_{w . x}+\frac{l_{b . w}}{2} \cdot \sin \left(\phi_{y}\right) \\ p_{w . x}-\frac{l_{b . w}}{2} \cdot \cos \left(\phi_{y}\right) \\ p_{w . z}\end{array}\right]$

$\left[\begin{array}{l}p_{b . x} \\ p_{b . y} \\ p_{b . z}\end{array}\right]=\left[\begin{array}{ccc}p_{w . x} & + & l_{b . c 2 a} \cdot \sin \left(\phi_{x}\right) \cdot \cos \left(\phi_{y}\right) \\ p_{w . y} & + & l_{b . c 2 a} \cdot \sin \left(\phi_{x}\right) \cdot \sin \left(\phi_{y}\right) \\ p_{w . z} & + & l_{b . c 2 a} \cdot \cos \left(\phi_{x}\right)\end{array}\right]$ 
Typically, initial conditions are assumed to be as follows:

$$
\left[\begin{array}{l}
p_{w . x}(0) \\
p_{w . y}(0) \\
p_{w . z}(0)
\end{array}\right]=\left[\begin{array}{c}
0 \\
0 \\
r_{w}
\end{array}\right]
$$




\subsection{Differential Equations}

After creating a nonlinear model using the Lagrangian method, and then linearizing that model, Yamamoto [1] provides the differential equations (4.7) and (4.18), [and their abbreviated term definitions].

\subsubsection{Wheel Angular Position $\theta$ and Body Pitch $\phi_{x}$}

Equation (4.7) corresponds to wheel angular position $\theta$ and body pitch $\phi_{x}$.

$$
\begin{aligned}
& \mathbf{K}_{1 . \ddot{x}} \cdot\left[\begin{array}{c}
\ddot{\theta} \\
\ddot{\phi}_{x}
\end{array}\right]+\mathbf{K}_{1 . \dot{x}} \cdot\left[\begin{array}{c}
\dot{\theta} \\
\dot{\phi}_{x}
\end{array}\right]+\mathbf{K}_{1 . x} \cdot\left[\begin{array}{c}
\theta \\
\phi_{x}
\end{array}\right]=\mathbf{K}_{1 . v} \cdot\left[\begin{array}{l}
v_{m t r . l} \\
v_{m t r . r}
\end{array}\right] \\
& \mathbf{K}_{1 . \ddot{x}}=\left[\begin{array}{ll}
+k_{1.1} & +k_{1.2} \\
+k_{1.2} & +k_{1.3}
\end{array}\right] \\
& \mathbf{K}_{1 . \dot{x}}=\left[\begin{array}{ll}
+k_{1.4} & -k_{1.4} \\
-k_{1.4} & +k_{1.4}
\end{array}\right] \\
& \mathbf{K}_{1 . x}=\left[\begin{array}{cc}
0 & 0 \\
0 & +k_{1.5}
\end{array}\right] \\
& \mathbf{K}_{1 . v}=\left[\begin{array}{cc}
+k_{1.6} & +k_{1.6} \\
-k_{1.6} & -k_{1.6}
\end{array}\right] \\
& k_{1.1}=\left(2 \cdot m_{w}+m_{b}\right) \cdot r_{w}+J_{w} \\
& k_{1.2}=m_{b} \cdot r_{w} \cdot l_{b . c 2 a} \\
& k_{1.3}=m_{b} \cdot l_{b . c 2 a}^{2}+J_{b . \phi_{x}} \\
& k_{1.4}=2 \cdot\left(\frac{k_{m t r . T} \cdot k_{m t r . b E M F}}{R_{m t r}}+k_{f r . m 2 w}\right) \\
& k_{1.5}=-m_{b} \cdot a_{g} \cdot l_{b . c 2 a} \\
& k_{1.6}=\frac{k_{m t r . T}}{R_{m t r}}
\end{aligned}
$$




\subsubsection{Body Yaw $\phi_{y}$}

Equation (4.18) corresponds to body yaw $\phi_{y}$.

$$
\begin{aligned}
k_{2 . \ddot{x}} \cdot\left[\ddot{\phi}_{y}\right]+ & k_{2 . \dot{x}} \cdot\left[\dot{\phi}_{y}\right]=k_{2 . v} \cdot\left[v_{m t r . r}-v_{m t r . l}\right] \\
k_{2.0} & =\frac{l_{b . w}}{r_{w}} \\
k_{2 . \ddot{x}} & =\frac{1}{2} \cdot m_{w} \cdot l_{b . w}^{2}+\frac{1}{2} \cdot k_{2.0}^{2} \cdot J_{b . \phi_{y}} \\
k_{2 . \dot{x}} & =\frac{1}{2} \cdot k_{2.0}^{2} \cdot k_{1.4} \\
k_{2 . v} & =\frac{1}{2} \cdot k_{2.0} \cdot k_{1.6}
\end{aligned}
$$




\subsection{State-Space Representation}

The general form of state-space representation is exhibited in Equation (4.23).

$$
\begin{aligned}
\underset{n x 1}{\dot{\mathbf{x}}}=\underset{n x n}{\mathbf{A}} \cdot \underset{n x 1}{\mathbf{x}}+\underset{n x p}{\mathbf{B}} \cdot \underset{p x 1}{\mathbf{u}} \\
\underset{m x 1}{\mathbf{y}}=\underset{m x n}{\mathbf{C}} \cdot \underset{n x 1}{\mathbf{x}}+\underset{m x p}{\mathbf{D}} \cdot \underset{p x 1}{\mathbf{u}}
\end{aligned}
$$

The designated $x$ states and $p$ inputs are exhibited in Equations (4.24) - (4.25).

$$
\begin{array}{r}
\underset{n x 1}{\mathbf{x}}=\left[\begin{array}{c}
\theta \\
\phi_{x} \\
\dot{\theta} \\
\dot{\phi}_{x} \\
\phi_{y} \\
\dot{\phi}_{y}
\end{array}\right] \\
\underset{p x 1}{\mathbf{u}}=\left[\begin{array}{c}
v_{m t r . l} \\
v_{m t r . r}
\end{array}\right]
\end{array}
$$


The derivation of indices for the system matrices $\mathbf{A}$ and $\mathbf{B}$ which are nonintuitive are derived from Equations (4.7) - (4.18). in Equations (4.26) - (4.27).

$$
\begin{aligned}
& \mathbf{K}_{1 . \ddot{x}} \cdot\left[\begin{array}{c}
\ddot{\theta} \\
\ddot{\phi}_{x}
\end{array}\right]+\mathbf{K}_{1 . \dot{x}} \cdot\left[\begin{array}{c}
\dot{\theta} \\
\dot{\phi}_{x}
\end{array}\right]+\mathbf{K}_{1 . x} \cdot \cdot\left[\begin{array}{c}
\theta \\
\phi_{x}
\end{array}\right]=\mathbf{K}_{1 . v} \cdot \cdot\left[\begin{array}{l}
v_{m t r . l} \\
v_{m t r . r}
\end{array}\right] \\
& {\left[\begin{array}{c}
\ddot{\theta} \\
\ddot{\phi}_{x}
\end{array}\right]=\underbrace{-\mathbf{K}_{1 . \ddot{x}}^{-1} \cdot \mathbf{K}_{1 . \dot{x}}}_{\mathbf{A}_{1}} \cdot\left[\begin{array}{c}
\dot{\theta} \\
\dot{\phi}_{x}
\end{array}\right]+\underbrace{-\mathbf{K}_{1 . \ddot{x}}^{-1} \cdot \mathbf{K}_{1 . x}}_{\mathbf{A}_{0}} \cdot\left[\begin{array}{c}
\theta \\
\phi_{x}
\end{array}\right]+\underbrace{\mathbf{K}_{1 . \ddot{x}}^{-1} \cdot \mathbf{K}_{1 . v}}_{\mathbf{B}_{1}} \cdot\left[\begin{array}{c}
v_{m t r . l} \\
v_{m t r . r}
\end{array}\right]} \\
& k_{2 . \ddot{x}} \cdot\left[\ddot{\phi}_{y}\right]+\quad k_{2 . \dot{x}} \cdot\left[\dot{\phi}_{y}\right]=k_{2 . v} \cdot\left[v_{m t r . r}-v_{m t r . l}\right] \\
& {\left[\ddot{\phi}_{y}\right]=\underbrace{-k_{2 . \ddot{x}}^{-1} \cdot k_{2 . \dot{x}}}_{A_{2}} \cdot\left[\dot{\phi}_{y}\right]+\underbrace{k_{2 . \ddot{x}}^{-1} \cdot k_{2 . v}}_{B_{2}} \cdot\left[v_{m t r . r}-v_{m t r . l}\right]}
\end{aligned}
$$

Note that $K_{1 . \ddot{x}}$ must be invertible to perform the second step in in Equation (4.26). The derivation for matrix invertibility and the proof that $K_{1 . \ddot{x}}$ is nonsingular [and is therefore invertible], are exhibited in Equations (4.28) - (4.31).

$$
\begin{gathered}
\underset{2 x 2}{\mathbf{X}}=\left[\begin{array}{ll}
+X_{(1,1)} & +X_{(1,2)} \\
+X_{(2,1)} & +X_{(2,2)}
\end{array}\right] \\
\mathbf{X}^{-1}=\frac{1}{\operatorname{det}(\mathbf{X})} \cdot \operatorname{adj}(\mathbf{X})=\frac{1}{X_{(1,1)} \cdot X_{(2,2)}-X_{(1,2)} \cdot X_{(2,1)}} \cdot\left[\begin{array}{ll}
+X_{(2,2)} & -X_{(2,1)} \\
-X_{(1,2)} & +X_{(1,1)}
\end{array}\right] \\
\operatorname{det}(\mathbf{X}) \neq 0 \\
\operatorname{det}\left(\mathbf{K}_{1 . \ddot{x}}\right)=k_{1.1} \cdot k_{1.3}-k_{1.2} \cdot k_{1.2} \neq 0
\end{gathered}
$$


The $\mathbf{A}$ matrix and the state vector $\mathbf{x}$ are exhibited in Equation (4.32).

$$
\underset{n x n}{\mathbf{A}} \cdot \underset{n x 1}{\mathbf{x}}=\left[\begin{array}{ccccccc}
0 & 0 & 1 & 0 & 0 & 0 \\
0 & 0 & 0 & 1 & 0 & 0 \\
\mathbf{A}_{0(1,1)} & \mathbf{A}_{0(1,2)} & \mathbf{A}_{1(1,1)} & \mathbf{A}_{1(1,2)} & 0 & 0 \\
\mathbf{A}_{0(2,1)} & \mathbf{A}_{0(2,2)} & \mathbf{A}_{1(2,1)} & \mathbf{A}_{1(2,2)} & 0 & 0 \\
0 & 0 & 0 & 0 & 0 & 1 \\
0 & 0 & 0 & 0 & 0 & A_{2}
\end{array}\right] \cdot\left[\begin{array}{c}
\theta \\
\phi_{x} \\
\dot{\theta} \\
\dot{\phi}_{x} \\
\phi_{y} \\
\dot{\phi}_{y}
\end{array}\right]
$$

The $\mathbf{B}$ matrix and the input vector $\mathbf{u}$ are exhibited in Equation (4.33).

$$
\underset{n x p}{\mathbf{B}} \cdot \underset{p x 1}{\mathbf{u}}=\left[\begin{array}{cc}
0 & 0 \\
0 & 0 \\
\mathbf{B}_{\mathbf{1}(\mathbf{1}, \mathbf{1})} & \mathbf{B}_{\mathbf{1}(\mathbf{1 , 2})} \\
\mathbf{B}_{\mathbf{1}(\mathbf{2}, \mathbf{1})} & \mathbf{B}_{\mathbf{1}(\mathbf{2}, \mathbf{2})} \\
0 & 0 \\
-B_{2} & +B_{2}
\end{array}\right] \cdot\left[\begin{array}{l}
v_{m t r . l} \\
v_{m t r . r}
\end{array}\right]
$$

The $\mathbf{C}$ matrix and the state vector $\mathbf{x}$ are exhibited in Equation (4.34).

$$
\underset{m x n}{\mathbf{C}} \cdot \underset{n x 1}{\mathbf{x}}=\left[\begin{array}{cccccc}
1 & 0 & 0 & 0 & 0 & 0 \\
0 & 1 & 0 & 0 & 0 & 0 \\
0 & 0 & 1 & 0 & 0 & 0 \\
0 & 0 & 0 & 1 & 0 & 0 \\
0 & 0 & 0 & 0 & 1 & 0 \\
0 & 0 & 0 & 0 & 0 & 1
\end{array}\right] \cdot\left[\begin{array}{c}
\theta \\
\phi_{x} \\
\dot{\theta} \\
\dot{\phi}_{x} \\
\phi_{y} \\
\dot{\phi}_{y}
\end{array}\right]
$$

The $\mathbf{D}$ matrix and the input vector $\mathbf{u}$ are exhibited in Equation (4.35).

$$
\underset{m x p}{\mathbf{D}} \cdot \underset{p x 1}{\mathbf{u}}=\left[\begin{array}{cc}
0 & 0 \\
0 & 0 \\
0 & 0 \\
0 & 0 \\
0 & 0 \\
0 & 0
\end{array}\right] \cdot\left[\begin{array}{l}
v_{m t r . l} \\
v_{m t r . r}
\end{array}\right]
$$




\subsection{Calculation of Nonintuitive Parameters}

Most of the hardware-equivalent dynamic model parameter values (with respect to the MinSeg hardware) were publicly available $[5,16]$, or were intuitive to obtain $\left[\right.$ Example: $\left.l_{h}, l_{w}, l_{d}\right]$.

Methods to determine those parameters which were not considered easily obtained are defined in the following sections.

\subsubsection{Moment of Inertia: Body: X-axis (Pitch) $J_{\phi_{x}}$}

The moment of inertia of the body with respect to pitch, is assumed to be sufficiently equivalent to the moment of inertia of "an ideal thin rectangular plate with length $l_{h}$, width $l_{w}=0$, an axis of rotation at one end of the plate".

This relation is exhibited in Equation (4.36).

$$
J_{\phi_{x}}=\frac{m_{b} \cdot l_{b . c 2 a}^{2}}{3}
$$

\subsubsection{Moment of Inertia: Body: Y-axis (Yaw) $J_{\phi_{y}}$}

The moment of inertia of the body with respect to pitch, is assumed to be sufficiently equivalent to the moment of inertia of "an ideal thin rectangular plate with length $l_{h}$, width $l_{w}=0$, an axis of rotation at one end of the plate".

This relation is exhibited in Equation (4.37).

$$
J_{\phi_{y}}=\frac{m_{b} \cdot\left(l_{b . w}^{2}+l_{b . d}^{2}\right)}{12}
$$




\subsubsection{Length From Body Center of Mass to Body Axis of Rotation $l_{b . c 2 a}$}

The length from the body center of mass to the body axis of rotation $l_{b . c 2 a}$ may be determined using more than one method.

\subsubsection{Yamamoto Method}

As seen in Figure 4.1 [on page 38], Yamamoto [1] assumes that the geometries of the wheels and the body are uniform. He also assumes that the masses of these geometries are uniform. He therefore defines length from the body center of mass to the body axis of rotation $l_{b . c 2 a}$, as exhibited in Equation (4.38)

$$
l_{b . c 2 a}=\frac{l_{b . h}}{2}
$$




\subsubsection{Vaccaro Method}

Since the geometries of the actual hardware are assumed to significantly deviate from the assumption of uniform mass distribution, an alternative method is instead used to calculate length from the body center of mass to the body axis of rotation $l_{b . c 2 a}$, as exhibited in Equation (4.38)

If the hardware is mounted at both wheel axles along the axis which is shared by both wheel axles, and if the hardware is given a degree of freedom to rotate about the wheel axle axis, without rotating the actual wheel axles, then the hardware may be lifted slightly and then released to swing freely like a pendulum along that axis.

Allowing the hardware to freely swing like a pendulum along the wheel axle axis significantly simplifies the dynamic equations of motion of the hardware. Furthermore, if friction at the newly added mount coupling points is negligible, then there will not be a need to model and implement the friction into the dynamics equations.

If the hardware is freely swung like a pendulum along the wheel axle axis as described above, then the relations exhibited in Equations (4.39) - (4.40) become true.

$$
\begin{aligned}
& \theta=\phi_{x} \\
& \mathbf{u}=\mathbf{0}
\end{aligned}
$$

The effects of these changes are exhibited in Equation (4.42) [on page 50]. This results in two relations, which are exhibited in Equation (4.41).

$$
\begin{array}{cc}
\ddot{\phi}_{x}+ & 0=0 \\
\ddot{\phi}_{x}+\underbrace{\frac{k_{1.5}}{k_{1.2}+k_{1.3}}}_{k_{\omega}} \cdot \phi_{x}=0 & =
\end{array}
$$

Of the two resulting relations in Equation (4.41), the former cannot be true while the hardware is in motion; thus, the latter is selected, as depicted on the right with a left-facing arrow. 


$$
\mathbf{K}_{1 . \ddot{x}} \cdot \underbrace{\left[\begin{array}{c}
\ddot{\theta} \\
\ddot{\phi}_{x}
\end{array}\right]+\mathbf{K}_{1 . \dot{x}}^{\left[\begin{array}{c}
\dot{\theta} \\
\dot{\phi}_{x}
\end{array}\right]+\mathbf{K}_{1 . x}} \cdot \underbrace{\left[\begin{array}{c}
\theta \\
\phi_{x}
\end{array}\right]}=\mathbf{K}_{1 . v} \cdot\left[\begin{array}{c}
v_{m t r . l} \\
v_{m t r . r}
\end{array}\right]}
$$$$
\underbrace{\mathbf{K}_{1 . \ddot{x}}} \cdot\left[\begin{array}{l}
\ddot{\phi}_{x} \\
\ddot{\phi}_{x}
\end{array}\right]+\underbrace{\mathbf{K}_{1 . \dot{x}}} \cdot\left[\begin{array}{l}
\dot{\phi}_{x} \\
\dot{\phi}_{x}
\end{array}\right]+\underbrace{\mathbf{K}_{1 . x}} \cdot\left[\begin{array}{l}
\phi_{x} \\
\phi_{x}
\end{array}\right]=\underbrace{\mathbf{K}_{1 . v} \cdot\left[\begin{array}{l}
0 \\
0
\end{array}\right]}
$$$$
\left[\begin{array}{ll}
k_{1.1} & k_{1.2} \\
k_{1.2} & k_{1.3}
\end{array}\right] \cdot\left[\begin{array}{l}
\ddot{\phi}_{x} \\
\ddot{\phi}_{x}
\end{array}\right]+2 \cdot k_{1.4} \cdot\left[\begin{array}{cc}
+1 & -1 \\
-1 & +1
\end{array}\right] \cdot\left[\begin{array}{c}
\dot{\phi}_{x} \\
\dot{\phi}_{x}
\end{array}\right]+\left[\begin{array}{cc}
0 & 0 \\
0 & k_{1.5}
\end{array}\right] \cdot\left[\begin{array}{l}
\phi_{x} \\
\phi_{x}
\end{array}\right]==\left[\begin{array}{l}
0 \\
0
\end{array}\right]
$$

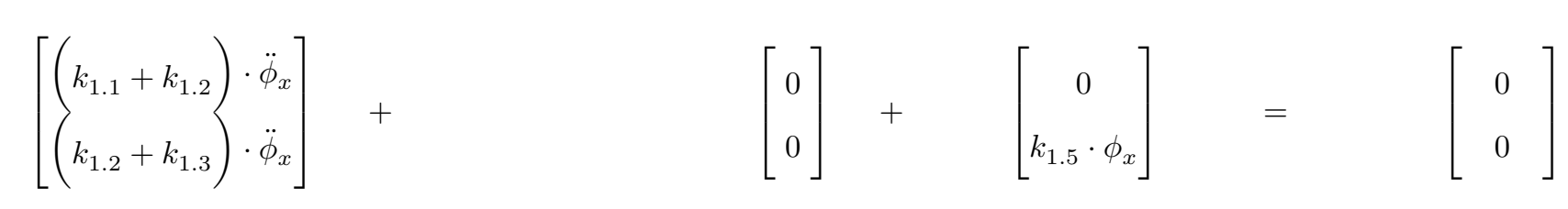


The coefficient term, abbreviated as $k_{w}$, is expanded in Equation (4.43). It may be expanded further with the use of Equation (4.36), as exhibited in Equation (4.44).

$$
\begin{gathered}
k_{\omega}=\frac{k_{1.5}}{k_{1.2}+k_{1.3}}=\frac{-m_{b} \cdot a_{g} \cdot l_{b . c 2 a}}{\left(m_{b} \cdot r_{w} \cdot l_{b . c 2 a}\right)+\left(m_{b} \cdot l_{b . c 2 a}^{2}+J_{b . \phi_{x}}\right)} \\
k_{\omega}=\frac{-m_{b} \cdot l_{b . c 2 a} \cdot a_{g}}{m_{b} \cdot l_{b . c 2 a} \cdot r_{w}+m_{b} \cdot l_{b . c 2 a}^{2}+\left(m_{b} \cdot l_{b . c 2 a}^{2} \cdot \frac{1}{3}\right)}=\frac{-a_{g}}{r_{w}+l_{b . c 2 a} \cdot\left(1+\frac{1}{3}\right)}
\end{gathered}
$$

\section{Harmonic Oscillator}

Notably, the selected relation in Equation (4.41) form-matches the equation for a harmonic oscillator [2, p. $119-120,122-123]$, as is exhibited in Equation (4.45).

$$
\begin{aligned}
& \ddot{y}+\omega^{2} \cdot y=\omega^{2} \cdot u \\
& \ddot{\phi}_{x}+k_{\omega} \cdot \phi_{x}=k_{\omega} \cdot 0
\end{aligned}
$$

This allows for the relation of the abbreviated term representing the system dynamics, $k_{w}$, to the natural angular frequency of the hardware [a pendulum] $\omega_{p}$, as is exhibited in Equation (4.46).

$$
\omega_{p}^{2}=k_{\omega}=\frac{-a_{g}}{r_{w}+l_{b . c 2 a} \cdot \frac{4}{3}}
$$

This proves significant since $\omega_{p}$ represents the angular frequency of the pendulum, which is a mea-

surable value, and since $k_{\omega}$ includes the desired unknown term $l_{b . c 2 a}$. [All other terms are known]. The relation may rewritten to solve for length from the body center of mass to the body axis of rotation $l_{b . c 2 a}$, as is exhibited as Equation (4.47).

$$
l_{b . c 2 a}=-\frac{3}{4} \cdot\left(\frac{a_{g}}{\omega_{p}^{2}}+r_{w}\right)=-\frac{3}{4} \cdot\left(\frac{a_{g}}{\left(2 \cdot \pi \cdot f_{p}\right)^{2}}+r_{w}\right)
$$




\subsection{Bibliography}

[1] Y. Yamamoto. (May 1, 2009). Nxtway-gs (self-balancing two-wheeled robot) controller design, [Online]. Available: https : //www . mathworks . com/matlabcentral/fileexchange/19147nxtway - gs - - self - balancing - two - wheeled - robot - controller - design (visited on 07/03/2017).

[2] R. J. Vaccaro, Digital Control: A State-space Approach, ser. Mcgraw Hill Series in Electrical and Computer Engineering. Mcgraw-Hill College, Jan. 1995, ISBN: 978-0070667815.

[5] B. Howard and L. Bushnell, "Enhancing linear system theory curriculum with an inverted pendulum robot," in 2015 International Conference on Computer Science and Mechanical Automation (CSMA), IEEE, Hangzhou, China, Oct. 2015. DOI: 10.1109/CSMA . 2015.63.

[16] P. "Philo" Hurbain. (May 15, 2017). Nxt motor internals, [Online]. Available: http://www . philohome.com/nxtmotor/nxtmotor.htm (visited on 07/03/2017).

[19] M. D. Peltier, "Trajectory control of a two-wheeled robot," Master's thesis, University of Rhode Island (URI): Department of Electrical Engineering, Jan. 2012. 


\section{CHAPTER 5.}

\section{Control Design}

\subsection{Additional Dynamics}

Additional dynamics may be incorporated into a state feedback regulating system in order to beneficially alter the response of the system in various respects.

\subsubsection{Background}

A state feedback regulating system is depicted in Figure 5.1. It contains the hardware plant as well as the inverted system feedback gains.

The additional dynamics are added in Figure 5.2. In the figure, the output vector of the plant is demuxed into its individual output components such that certain outputs may additionally be used as inputs to the additional dynamics state-space representation. This is represented using flags; connections exist between flags with equivalent labels. The outputs of the plant as well as the outputs of the additional dynamics are then muxed to form an output vector representing the output of a larger system.

Thus, the larger system [which includes the plant and the additional dynamics] may temporarily be considered as new plant, as depicted in Figure 5.3. It may therefore be expressed as a single state-space representation containing both systems. Thus, state-feedback regulation techniques may be used to control the system; however, the system response will now include any benefits which the additional dynamics provide. A representation of the larger system is depicted in Figure 5.4. Note the increase in the output vector.

From this point, the system depiction may be rearranged such that the input to the controller is on the left, while the plant outputs remain on the right. A sequential description of the process, and the figure number which corresponds to each step is provided below:

1. The feedback gains are separated into those with respect to the original plant outputs $x$ and those with respect to the additional dynamics $x . a$.

[Figure 5.5]

2. The gains are shifted such that they are forward facing.

[Figure 5.6] 
- In this configuration, the original plant may be separated from the components used to control it, including the additional dynamics.

[Figure 5.7]

3. The plant is shifted to the right side of the system depiction.

[Figure 5.8] 


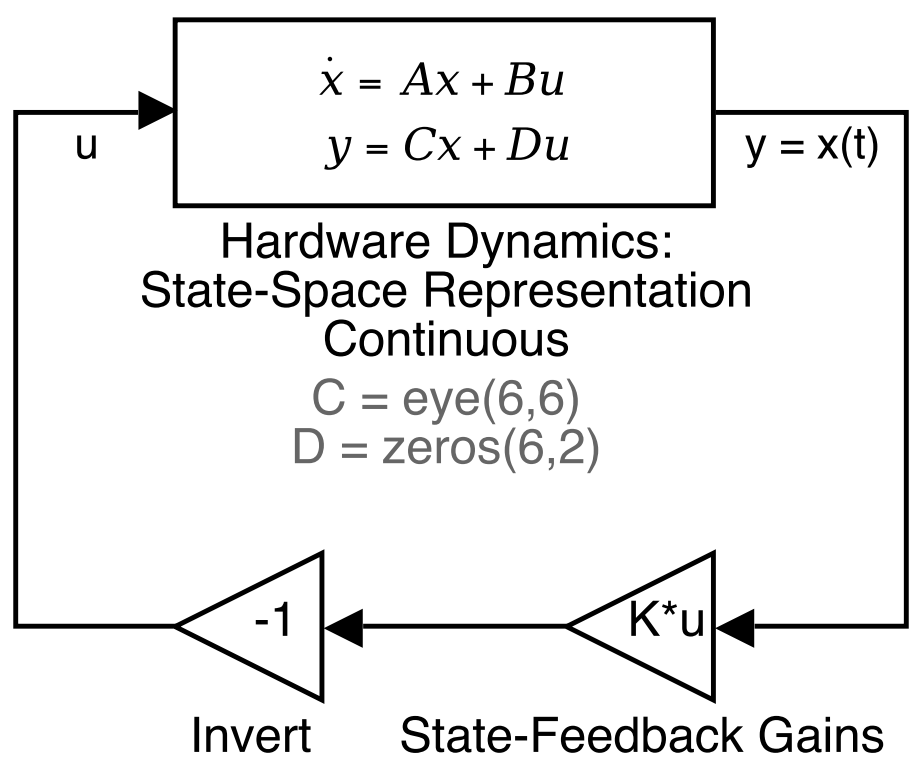

Figure 5.1.: [Additional Dynamics]: State Feedback Regulator 


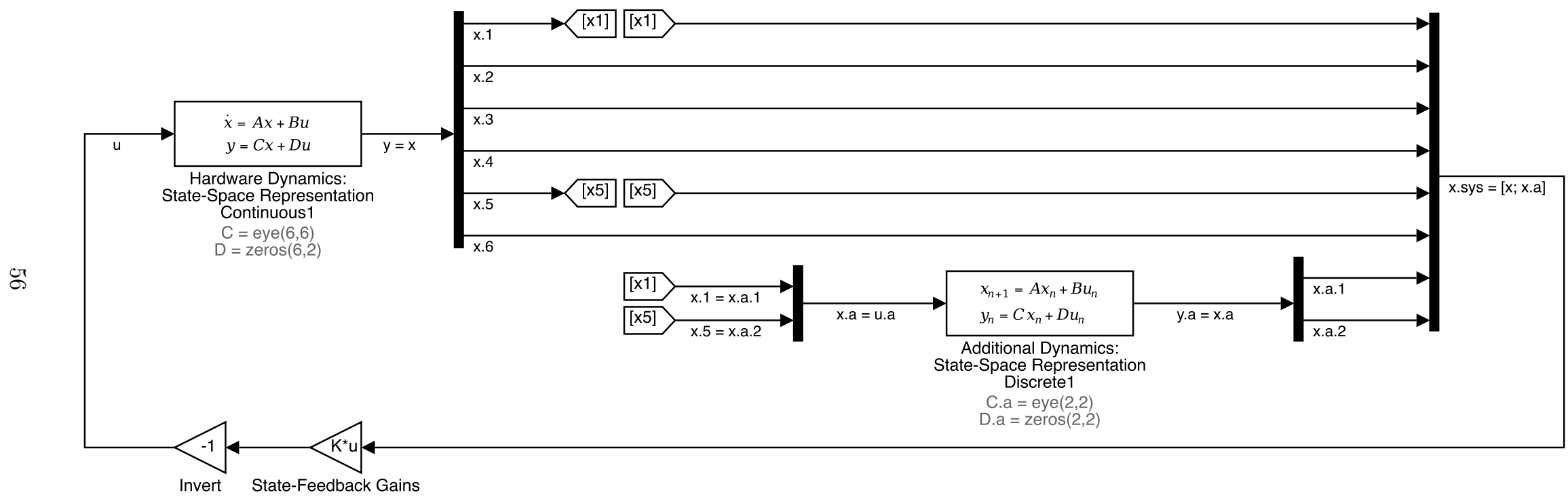

Figure 5.2.: [Additional Dynamics]: 1.0 Additional Dynamics (Design View) 


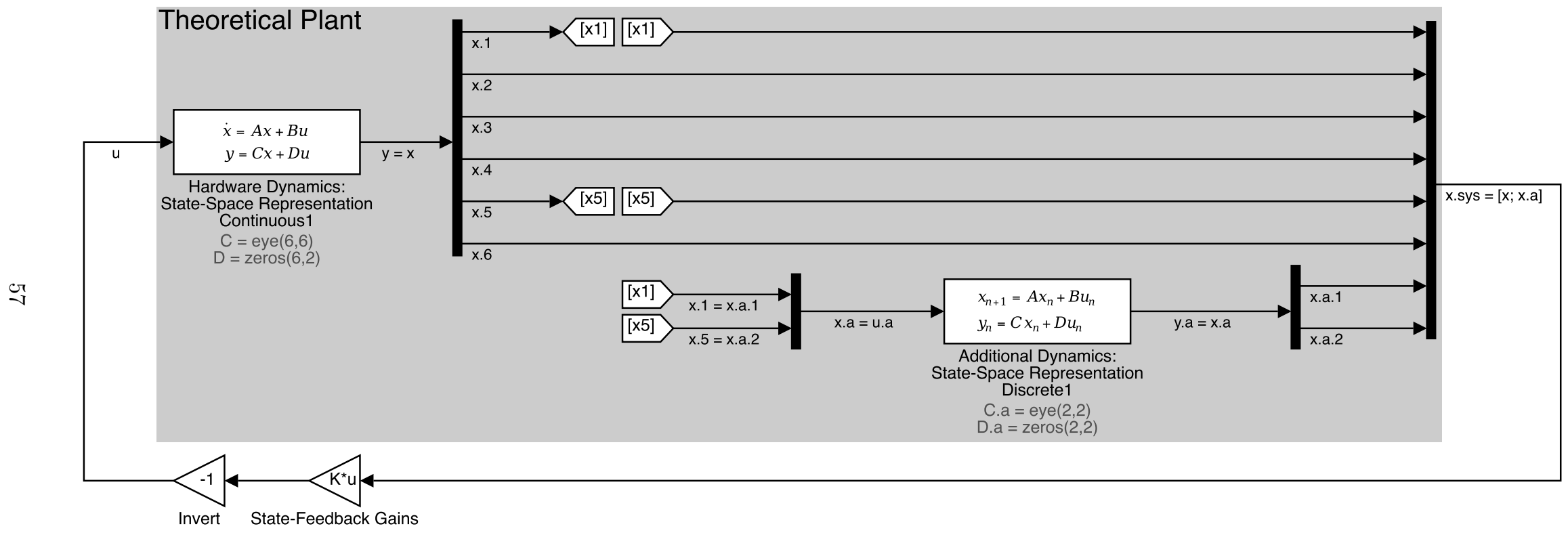

Figure 5.3.: [Additional Dynamics]: 1.1 Additional Dynamics (Design View) 


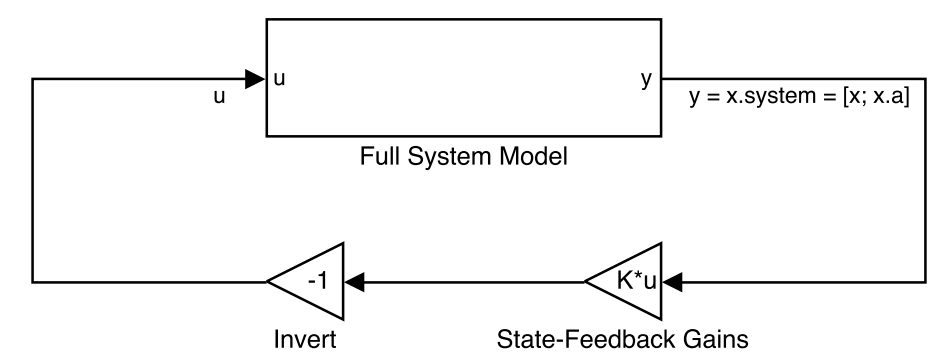

Figure 5.4.: [Additional Dynamics]: 1.2 Additional Dynamics (State Feedback Regulator View) 


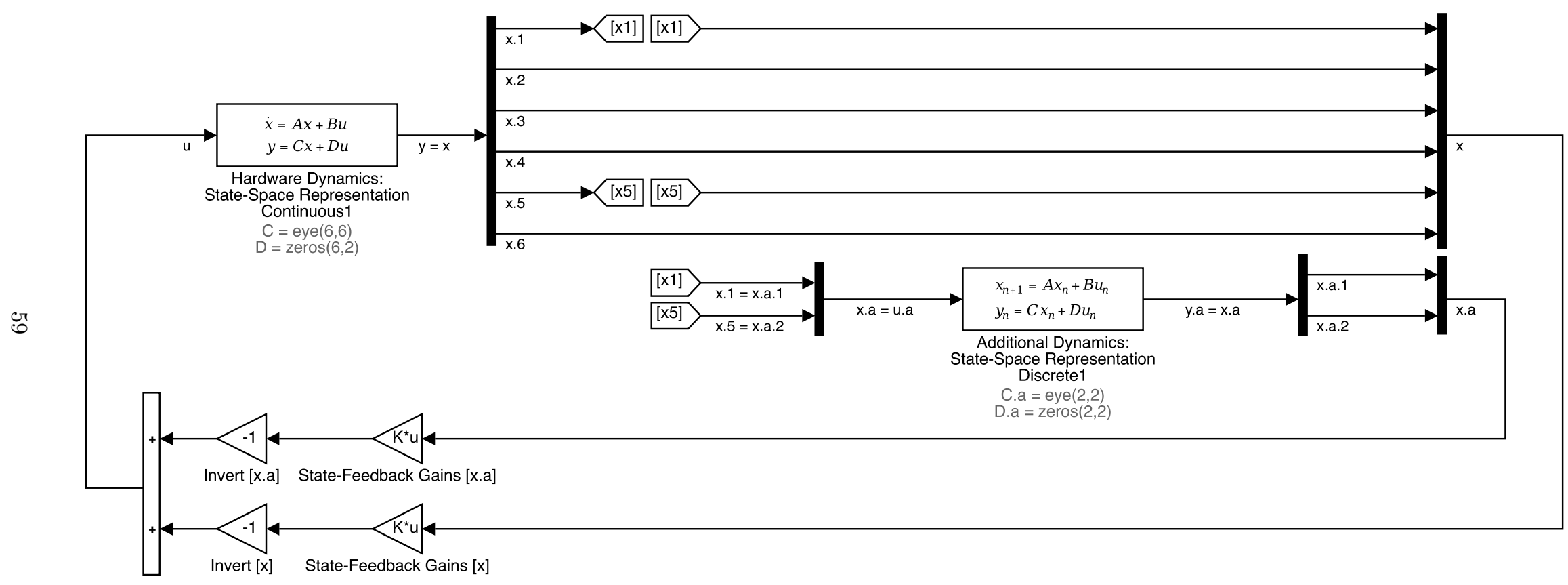

Figure 5.5.: [Additional Dynamics]: 2.0 Additional Dynamics (Split Gains) 


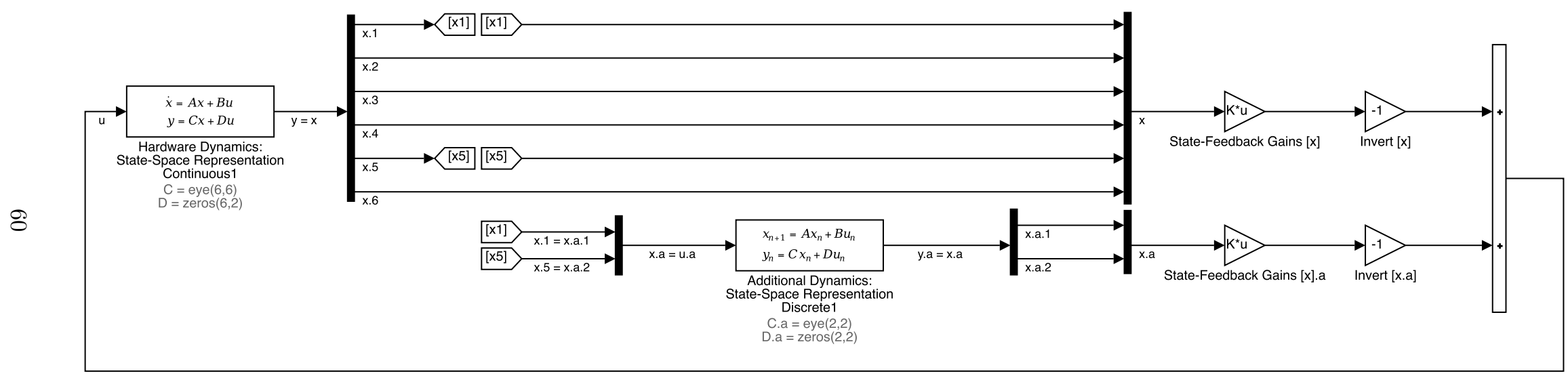

Figure 5.6.: [Additional Dynamics]: 3.0 Additional Dynamics (Linear View: Plant) 


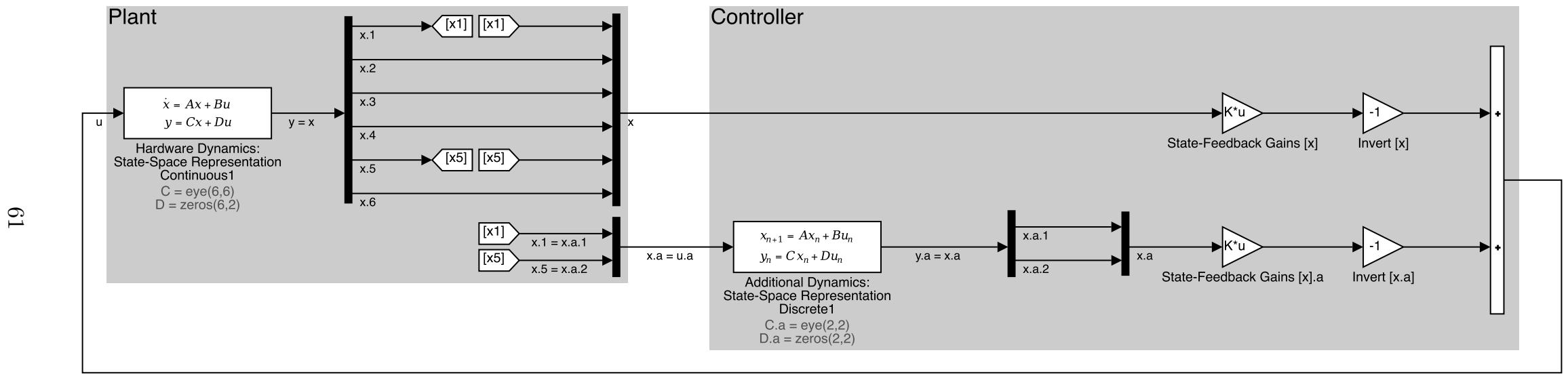

Figure 5.7.: [Additional Dynamics]: 3.1 Additional Dynamics (Linear View: Plant) 


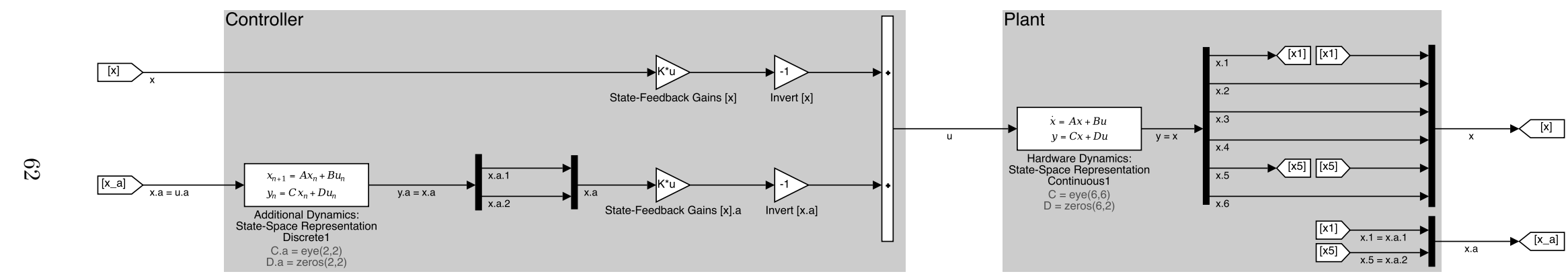

Figure 5.8.: [Additional Dynamics]: 4.0 Additional Dynamics (Linear View: Controller) 


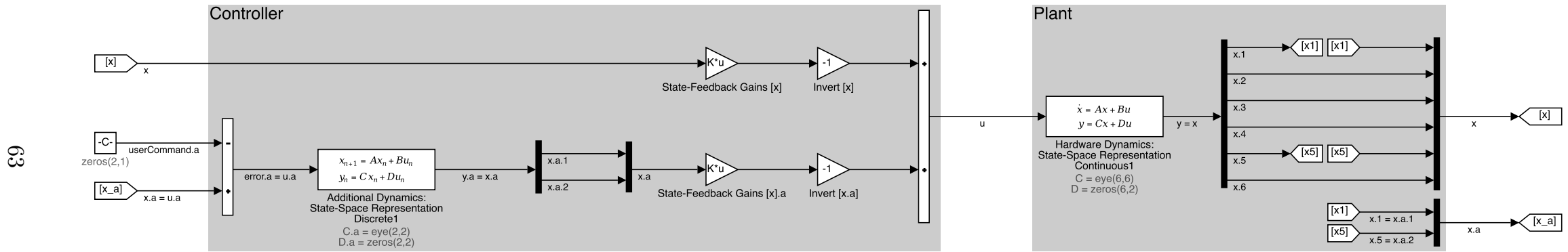

Figure 5.9.: [Additional Dynamics]: 4.0 Additional Dynamics with Reference Signal (Linear View: User) 


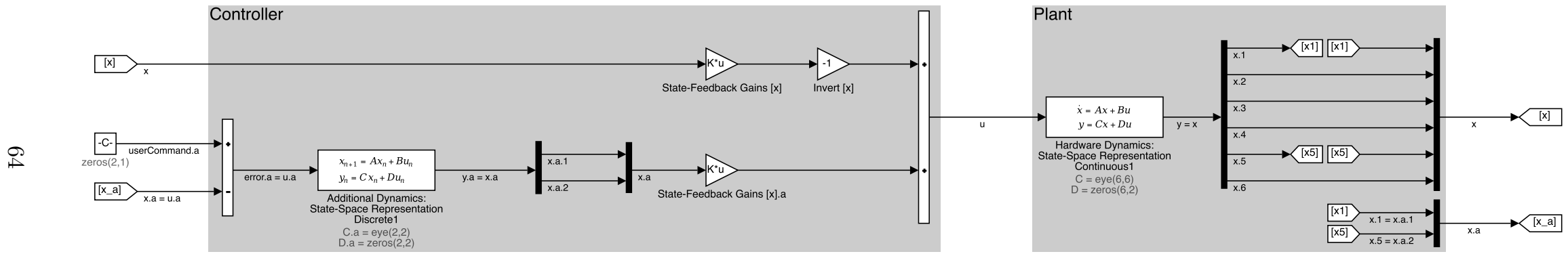

Figure 5.10.: [Additional Dynamics]: 4.0 Additional Dynamics with Reference Signal (Linear View: User) 


\subsubsection{Reference Signal}

Recall that a standard state-feedback regulator simply brings its inputs, $[$ in this case, system states $x$ and $x_{a}$ ], to zero. If it is desired that a controller input be brought to a value other than zero, a reference signal may be implemented.

In these cases, rather than input the controller with a state which the controller will bring to zero, the controller is input with the difference between the state value and the reference [desired] value. This difference is commonly known as the error signal. Once the error signal is brought to zero for a given state, the state will be equivalent to the desired reference value.

Returning to system depiction, a reference command is implemented, as depicted in Figure 5.9.

Note that the reference signal receives the negative. Inverting the system state alters the system equation, and could cause the system to become unstable.

Despite this fact, it is sometimes more common to see the system state subtracted from the reference signal. To correctly achieve this, once the reference signal is implemented, either side of the difference equation is multiplied by -1 . The negative on the input side is distributed to both inputs. The output of the difference equation is the input of the additional dynamics; thus, when the negative appears on the output side of the difference equation, a negative exists on either side of the additional dynamics equation.

Recall that all state-space representations are linear; therefore, the input and the output may be multiplied by the same value. In this case, the negative may be divided out on both sides.

These changes are depicted in Figure 5.10. 


\subsubsection{Tracking System}

Additional dynamics may be incorporated to improve reference tracking. When implemented for this purpose, the additional dynamics are known as a tracking system.

In the case of a tracking system, an integrator may be implemented as the additional dynamics to track a constant reference exactly, or to track a slowly varying reference approximately.

Integrators are also able to mitigate constant disturbances. Incidentally, the MinSeg M2V3 system uses gyroscopes as body angular velocity $\psi$ sensors. Bias is inherent in the output of a gyroscope; therefore, the use of such an integrator as a tracking system has an additional benefit: it will mitigate the effects of bias from a gyroscope output, whether directly or within terms which are derivative of the gyroscope output.

Thus, in the case of the two-wheeled robot, integrators are implemented as additional dynamics for the states representing wheel angular position $\theta$ and body angular position (yaw) $\phi_{y}$. This establishes a tracking system, [an augmented method of state feedback regulation], for the system. The state-space representation of the integrator is exhibited in Equation (5.1).

$$
\begin{aligned}
& \underset{n x 1}{\dot{\mathbf{x}}}=\left[\begin{array}{ll}
0 & 0 \\
0 & 0
\end{array}\right] \cdot \underset{n x 1}{\mathbf{x}}+\left[\begin{array}{ll}
1 & 0 \\
0 & 1
\end{array}\right] \cdot\left[\begin{array}{l}
e_{\theta} \\
e_{\phi_{y}}
\end{array}\right] \\
& \underset{m x 1}{\mathbf{y}}=\left[\begin{array}{ll}
1 & 0 \\
0 & 1
\end{array}\right] \cdot \underset{n x 1}{\mathbf{x}}+\left[\begin{array}{ll}
0 & 0 \\
0 & 0
\end{array}\right] \cdot\left[\begin{array}{l}
e_{\theta} \\
e_{\phi_{y}}
\end{array}\right]
\end{aligned}
$$




\subsubsection{Discrete Additional Dynamics}

Since the additional dynamics will be processed on a microcontroller, the additional dynamics will be digital; thus, a continuous-to-discrete conversion will be necessary. An integrator is an established case which is exhibited in Equation (5.2).

$$
\begin{aligned}
& \underset{n x 1}{\dot{\mathbf{x}}}=\left[\begin{array}{ll}
1 & 0 \\
0 & 1
\end{array}\right] \cdot \underset{n x 1}{\mathbf{x}}+\left[\begin{array}{ll}
1 & 0 \\
0 & 1
\end{array}\right] \cdot\left[\begin{array}{l}
e_{\theta} \\
e_{\phi_{y}}
\end{array}\right] \\
& \underset{m x 1}{\mathbf{y}}=\left[\begin{array}{ll}
1 & 0 \\
0 & 1
\end{array}\right] \cdot \underset{n x 1}{\mathbf{x}}+\left[\begin{array}{ll}
0 & 0 \\
0 & 0
\end{array}\right] \cdot\left[\begin{array}{l}
e_{\theta} \\
e_{\phi_{y}}
\end{array}\right]
\end{aligned}
$$




\subsubsection{Control Gains}

Once the additional dynamics are established, state feedback gains must be calculated. Multiple methods exist to calculate these gains. The most established methods involve optimization or poleplacement.

\subsubsection{Optimal}

Several optimal control techniques exist [20]. This section will focus on linear quadratic regulation techniques.

\subsection{Implementation}

In order to determine the feedback gains of the system, the state-space representation of the system, [the plant and the additional dynamics], is input into a discrete linear quadratic regulator gain-calculation Matlab function, $d l q r$, which outputs state-feedback gains which best minimize the quadratic cost function. The Matlab function also requires quadratic cost function matrices $Q$ and $R$ as inputs.

The quadratic cost matrices $Q$ and $R$ were determined through trial and error; however, some constraints existed. The $Q$ and $R$ matrices were both diagonal matrices; [thus, all indices which are not on the diagonal are equal to zero]. Also, the $R$ matrix was left as an identity matrix until the $Q$ matrix established desirable behavior. Once desirable behavior was established, the option of multiplying the $R$ matrix by a scalar value [greater than one] became a consideration.

Multiplying the $R$ matrix by a scalar value decreases the response time of the controller; however, this also decreases the peak magnitude of the control output, [in this case, motor voltage]. While a decreased response time is generally undesirable, the reduction of the control output can be necessary in certain circumstances. For example, the maximum permissible value for the control output, motor voltage, is limited by the nominal voltage provided by the hardware power source. 


\subsection{Results: Simulation}

To demonstrate the capabilities of the device, a dynamic command is provided which attempts to move the device in the shape of an eight 8 on the ground while maintaining balance.

Additionally, the device starts at a body angular position (pitch) $\phi_{x}$ of $0.03[\mathrm{rad}]$. This represents the inability to start the device at a perfect angle. This causes additional transients in the initial milliseconds of operation.

Figures 5.11 - 5.17 depict the system state during its operation while completing its response to a figure-eight linear position command. 

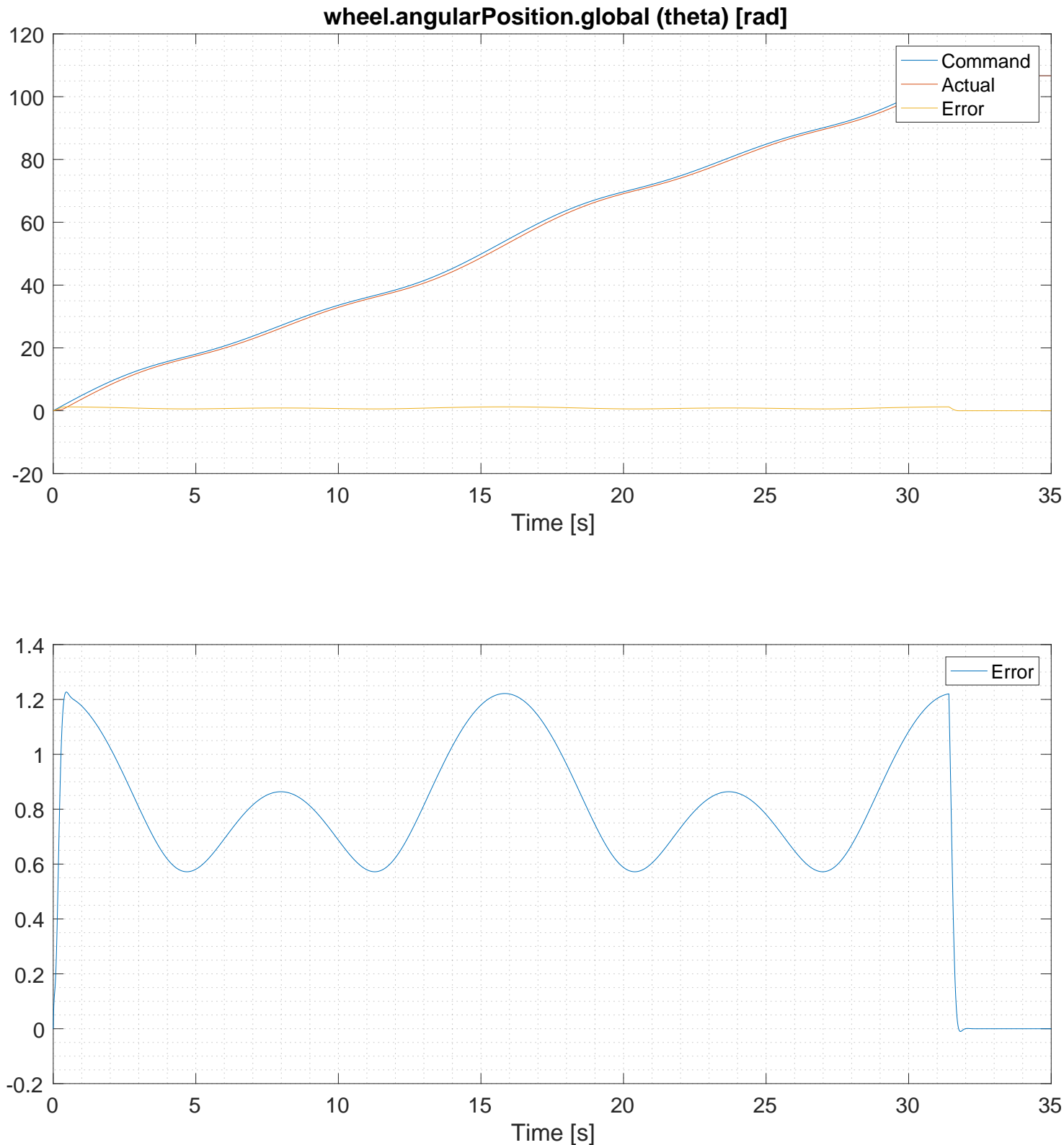

Figure 5.11.: [Control Gains: LQR]: Simulation Results: Wheel Angular Position $\theta$ 

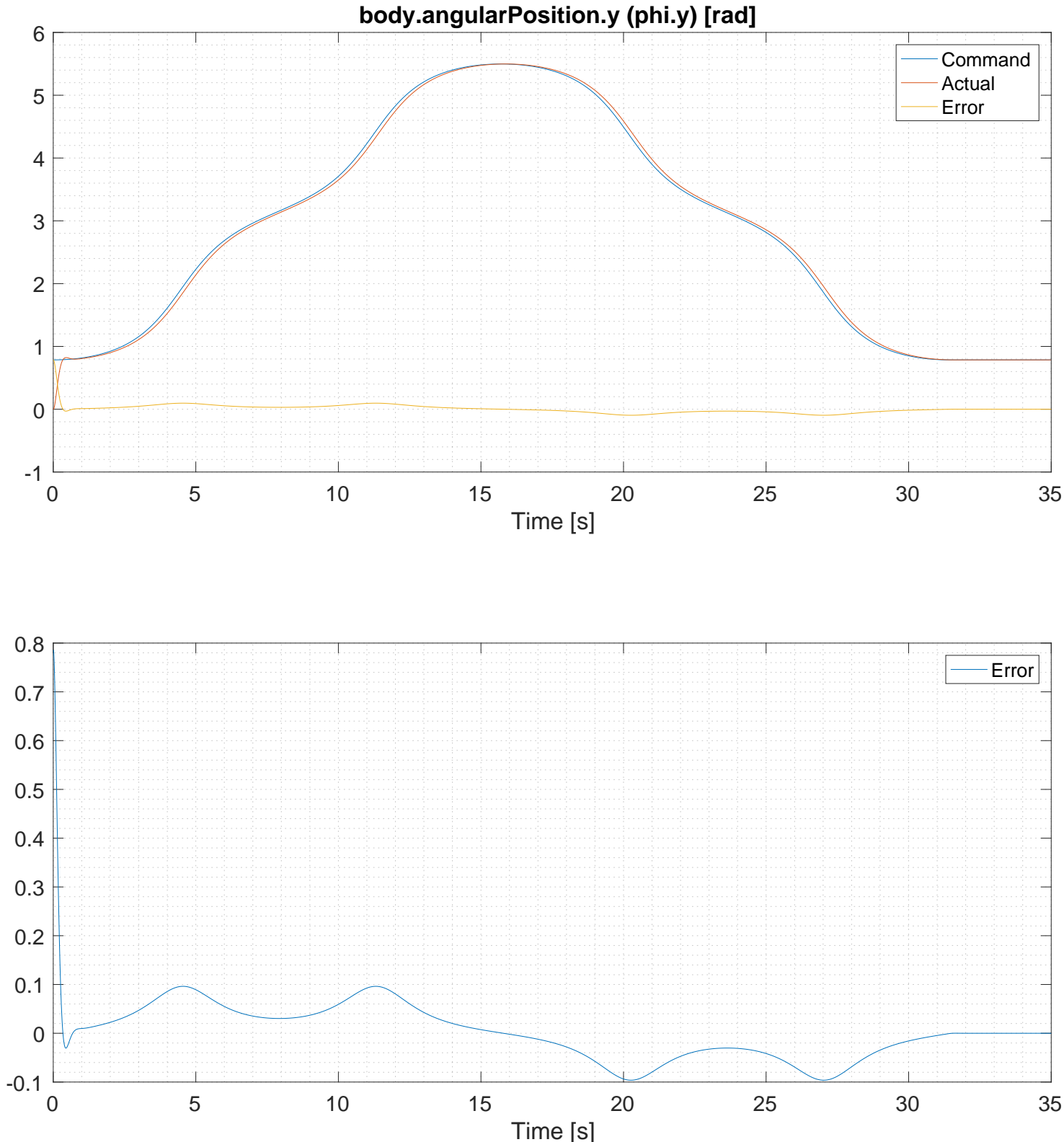

Figure 5.12.: [Control Gains: LQR]: Simulation Results: Body Angular Position $\phi_{y}$ 


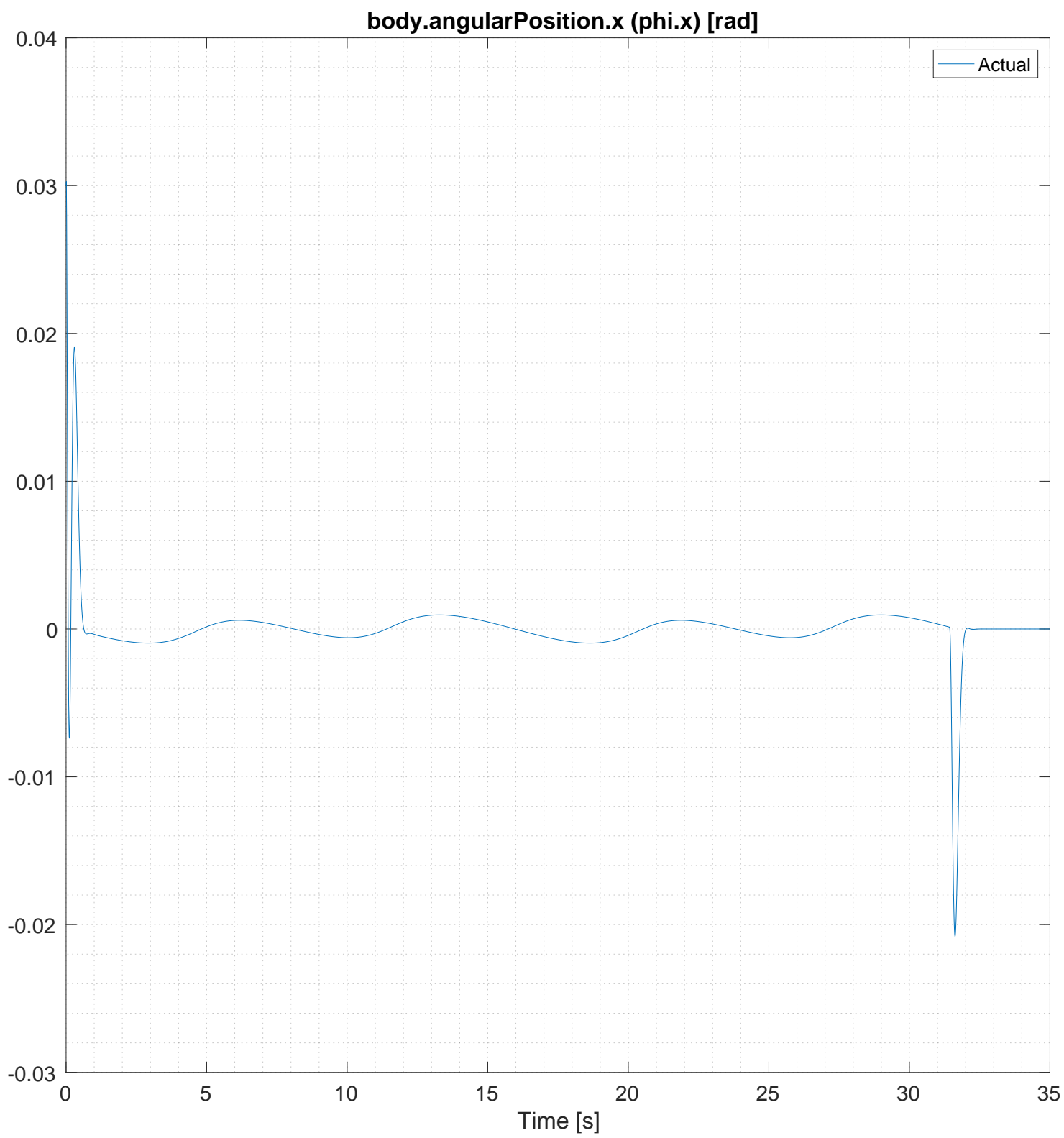

Figure 5.13.: [Control Gains: LQR]: Simulation Results: Body Angular Position $\phi_{x}$ 

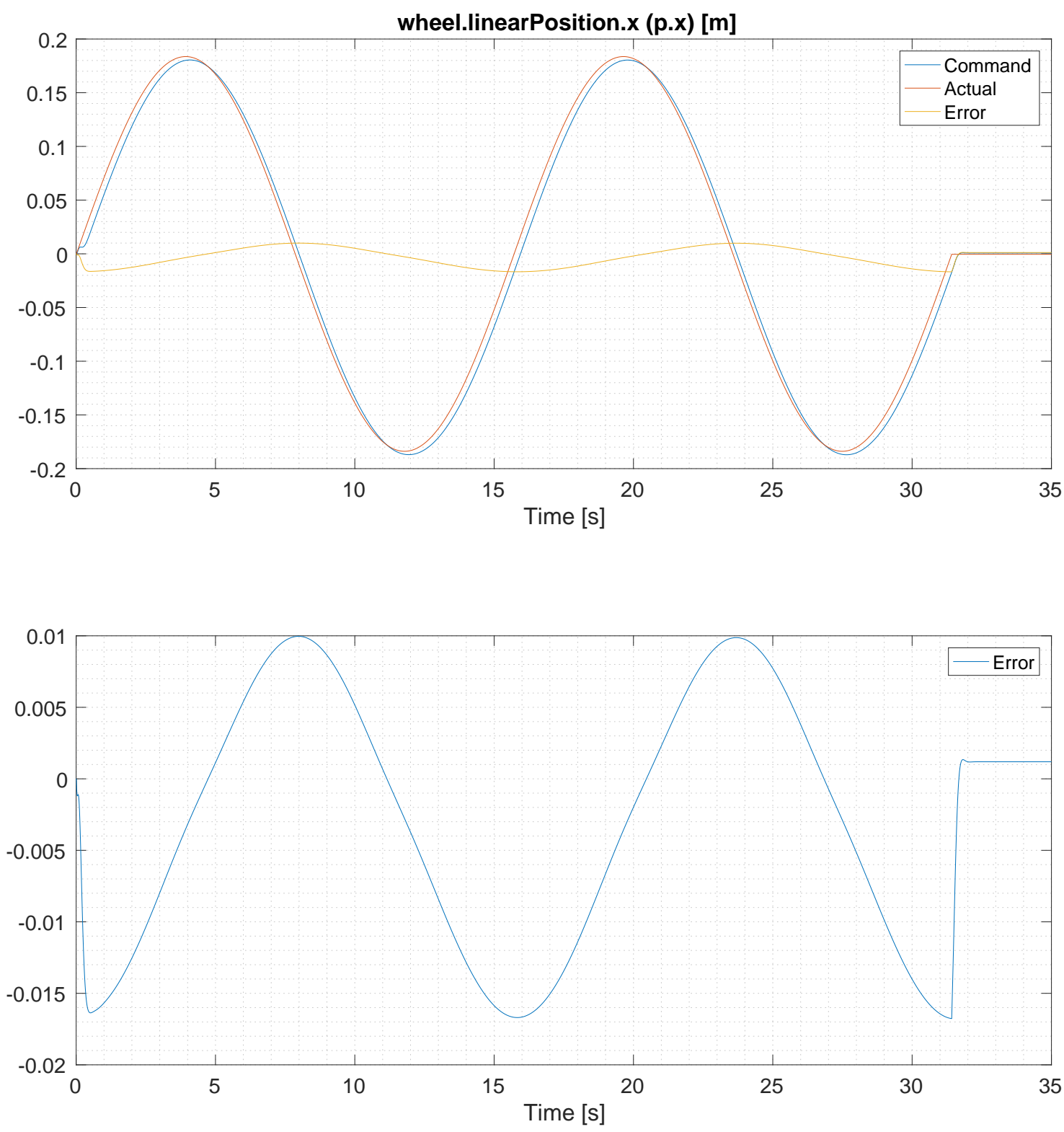

Figure 5.14.: [Control Gains: LQR]: Simulation Results: Wheel Linear Position $p_{x}$ 

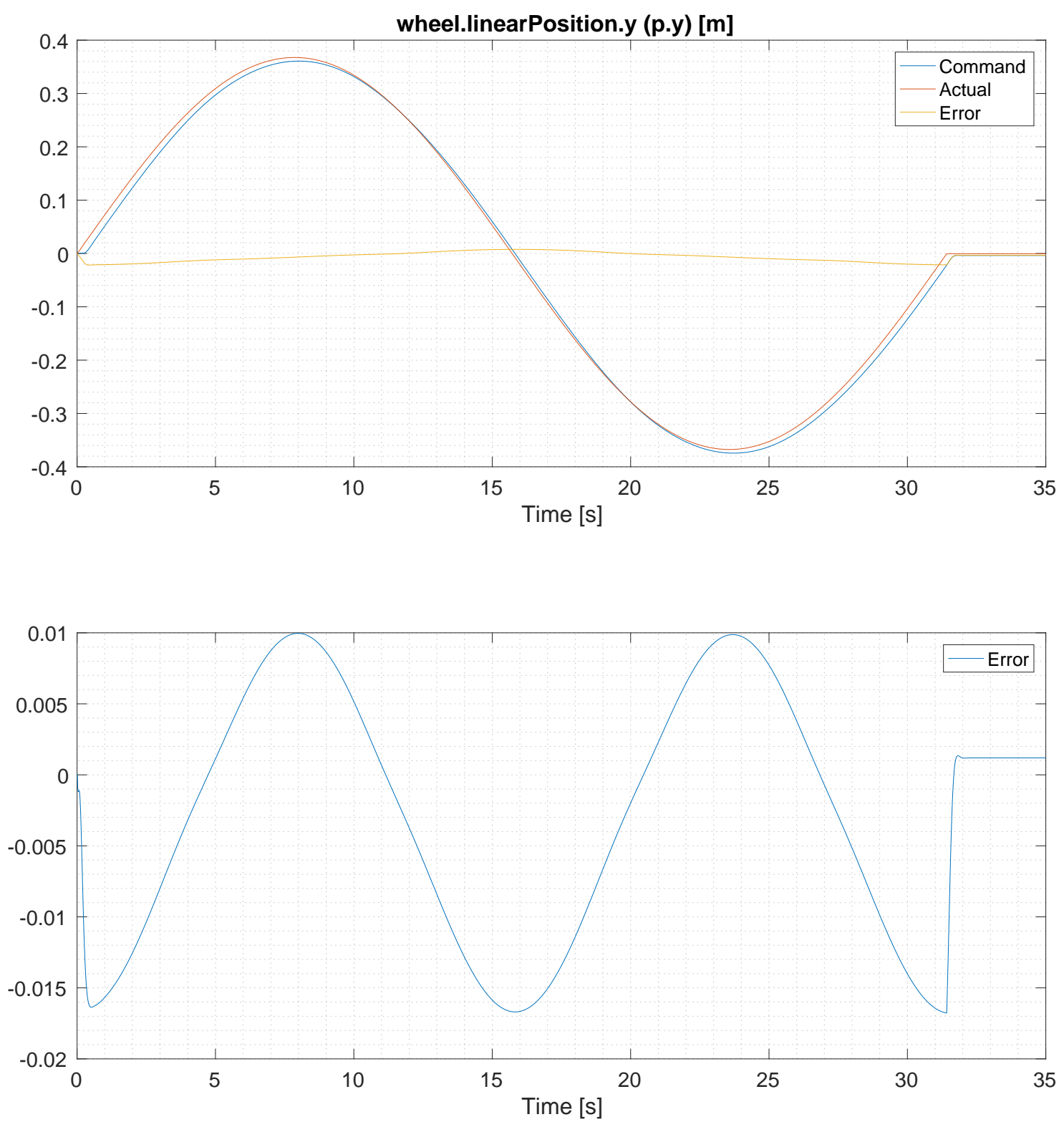

Figure 5.15.: [Control Gains: LQR]: Simulation Results: Wheel Linear Position $p_{y}$ 


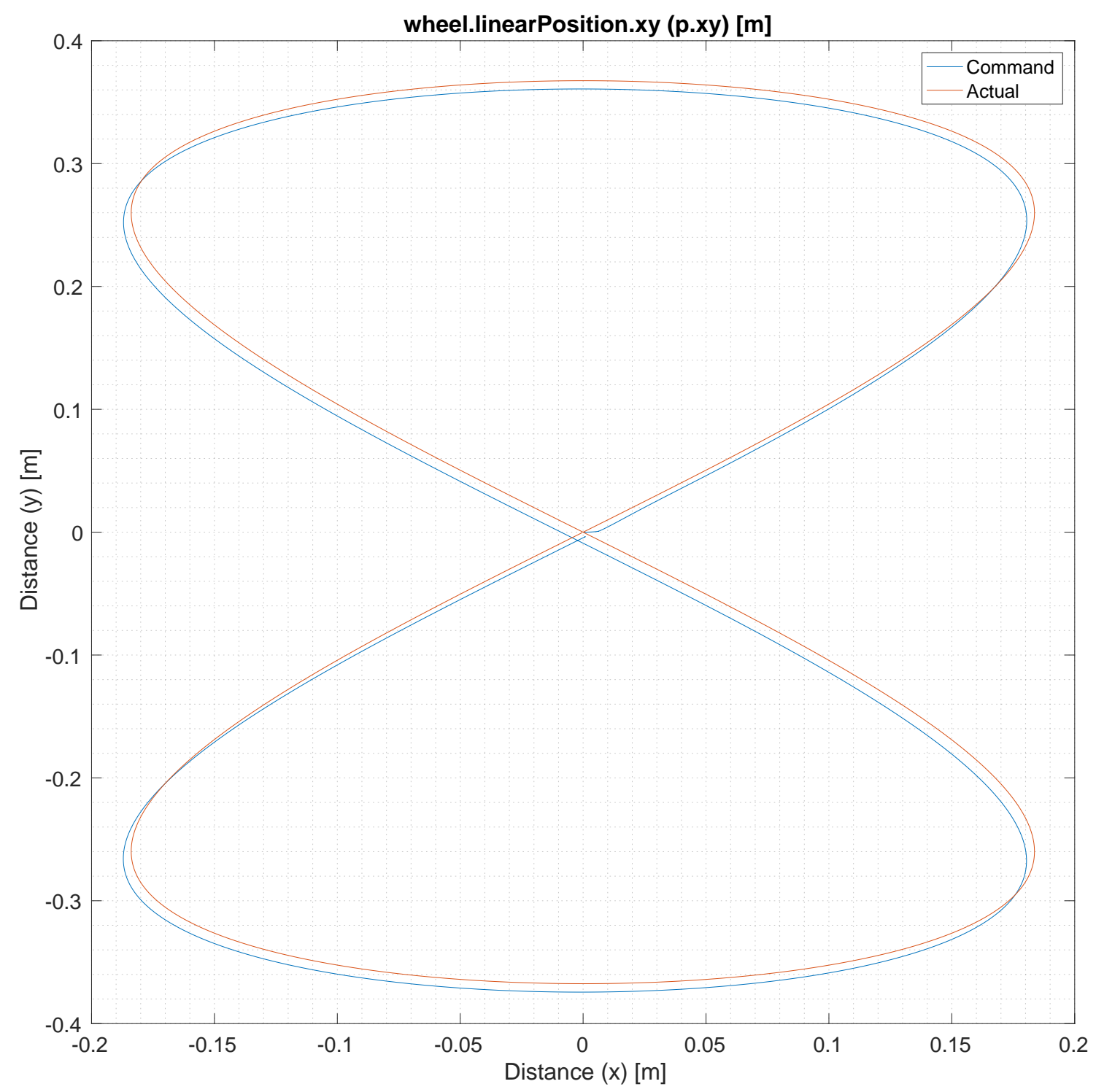

Figure 5.16.: [Control Gains: LQR]: Simulation Results: Wheel Linear Position $p_{x y}$ 


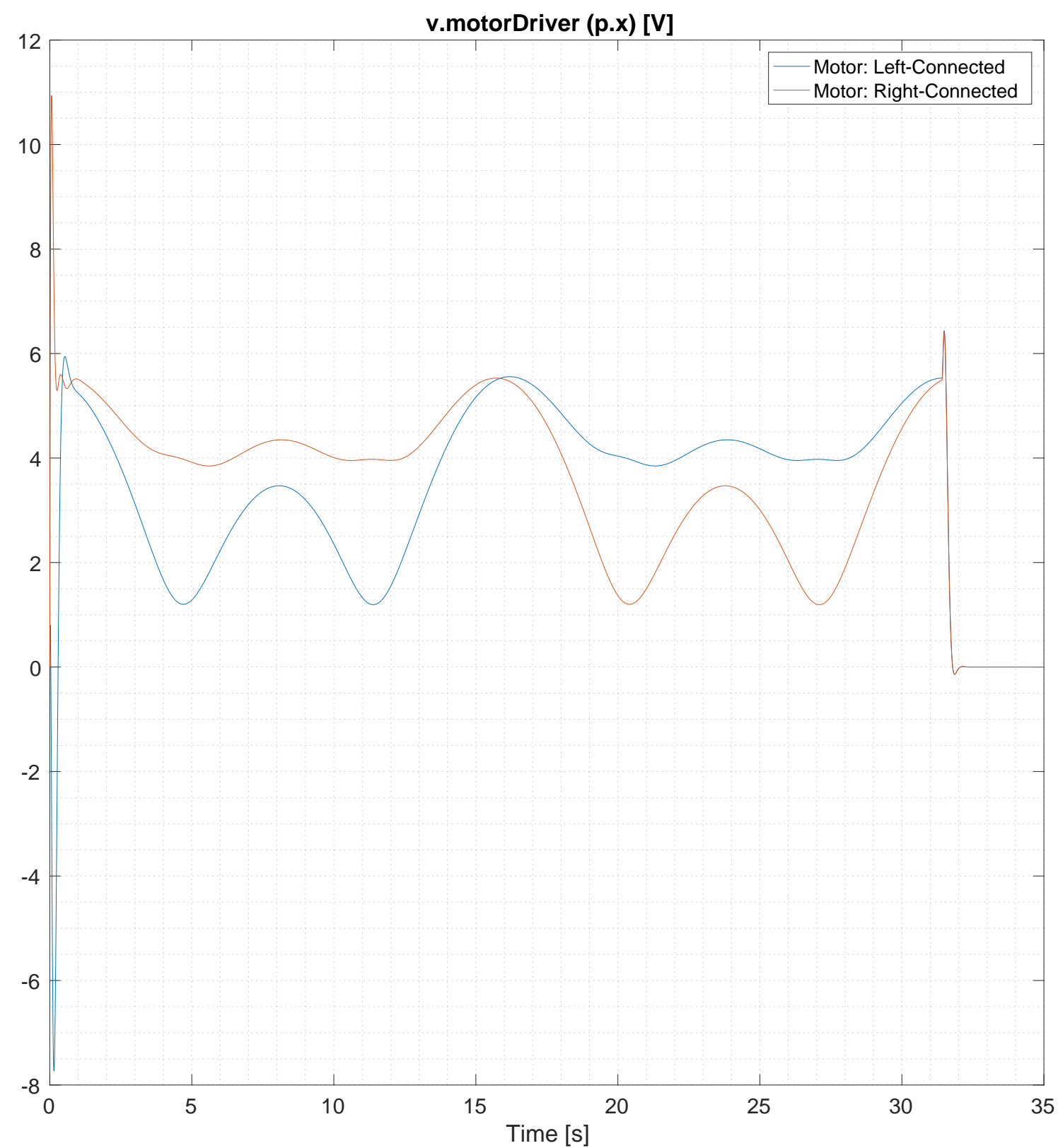

Figure 5.17.: [Control Gains: LQR]: Simulation Results: Motor Driver Commanded Voltage $v_{\text {motorDriver }}$ 


\subsection{Bibliography}

[20] F. L. Lewis and V. L. Syrmos, Optimal Control, Second. Wiley-Interscience, Nov. 1995, ISBN: 978-0-471-03378-3. 


\section{CHAPTER 6.}

\section{Conclusions}

The results provided in Section 5.1.3.1.2, demonstrate that it is theoretically possible to adequately track dynamic state commands specified to the two-wheeled robot system with respect to wheel angular position $\theta$ and body angular position (yaw) $\phi_{y}$, while maintaining standard regulation of all other plant states.

While moving in accordance with the dynamic commands provided to the wheel angular position

$\theta$ and body angular position (yaw) $\phi_{y}$ states, the robot remains balances and does not deviate significantly from the commands at any time.

\subsection{Future work}

The following is a non-comprehensive list of potential future work which could be performed to improve the capabilities of the test platform or supplement the control studies already performed on the test platform.

- Optimize sample interval of hardware.

- Determine limiting factors in the reduction of the board sampling interval.

- Improve upon these factors, if possible.

- Determine alternative model algorithms such that processing (per sample interval) is significantly minimized.

[Example: Use binary classes wherever possible.]

- Optimize serial communication.

- Implement bluetooth wireless transmission.

- Determine limiting factors in the reduction of the serial transmission interval. 
- Determine if increasing the serial communication sample interval improves limits on hardware sample interval.

This is already performed when sending a high number of signals,

but improved performance has not been verified.

- Determine if increasing the BAUD frequency on the board will remove limits on the serial transmission interval.

- Determine how to begin a read without resetting the hardware.

- Determine if dynamic/real-time plotting is worthwhile. If so, implement. 
- Implement alternate linear controllers.

- Implement pole-placement controller(s).

- Implement LQG controller(s).

- Implement $H_{N}$ controller(s).

[Where $N$ is an integer or infinity.]

- Implement a nonlinear plant model.

- Develop nonlinear controller(s).

- Demonstrate operation in nonlinear states.

Example: Operating in a state with a significantly increased component of horizontal pitch.

- Improve model parameters measurements.

- Improve mass measurements.

- Use scale with improved precision.

[Current precision is 0.01 [lb].]

- Improve motor transfer function measurement.

[Angular velocity vs. Input Voltage]

Hardware must remain perfectly upright while in motion to perform this measurement.

The original measurement was taken while balancing the in-motion device by hand.

Since a pitch controller has been developed, the measurement may be taken more accurately.

- Verify conflicting motor parameters derived from References [5], [16]:

- Resistance, $R_{m t r}$

- Torque constant, $k_{m t r . T}$

- Back EMF constant, $k_{m t r . b E M F}$

- Increase MinSeg power-source voltage-maximum.

[See Section 2.2.1.4.]

Current voltage source maximum: $09[\mathrm{~V}]$.

Motor driver operating maximum: 36 [V]. 
- Construct alternate physical models via simple variants.

- Alternate mass distribution.

- Reduce number of batteries to less than 6 .

[Requires use of USB cable for power.]

- Alternate geometry.

- Alternate wheel component.

[Search for Lego tires with differing radius, mass, and/or coefficient of friction. See [21]]

- Incorporation of a second mass on the pendulum.

- Perform movement on an uneven surface.

- Optimize filter design.

- Determine tradeoffs between no filter vs $1^{\text {st }}$ to $6^{\text {th }}$ order bessel filters.

- Determine tradeoffs between state-space and transfer function blocks, if any.

- Determine tradeoffs between Matlab besself and bessel poles, if any?

- Optimization of observable data

- Implement voltage sensor across battery holster.

Use this voltage reading to determine the true voltage of the power source in operation.

- Incorporate use of accelerometer?

Incorporate use of Kalman filter?

Compare effects. 
- Test Windows and Linux compatibility.

- Improve overrun detection.

If the board cannot complete all of its processes before the sampling interval completes,

then it performs incorrectly. Detection of this is possible and desirable for the user.

Currently, overrun detection requires that the the user manually view an LED on the board.

The LED is very small and almost entirely masked by the bluetooth module.

(Simulink also currently prevents status reads of the overrun LED pin.)

An alternative method should exist which alert the user more conveniently. 


\subsection{Bibliography}

[5] B. Howard and L. Bushnell, "Enhancing linear system theory curriculum with an inverted pendulum robot," in 2015 International Conference on Computer Science and Mechanical Automation (CSMA), IEEE, Hangzhou, China, Oct. 2015. DOI: 10.1109/CSMA.2015.63.

[16] P. "Philo" Hurbain. (May 15, 2017). Nxt motor internals, [Online]. Available: http://www . philohome.com/nxtmotor/nxtmotor.htm (visited on 07/03/2017).

[21] (Oct. 3, 2016). Lego wheels chart, [Online]. Available: http://wheels.sariel.pl/ (visited on $07 / 03 / 2017)$. 
Appendices 
APPENDIX A.

\section{Code Listings}

\section{A.1. $\operatorname{minseg.m}$}

Code Listing A.1: [minseg.m]: Root file

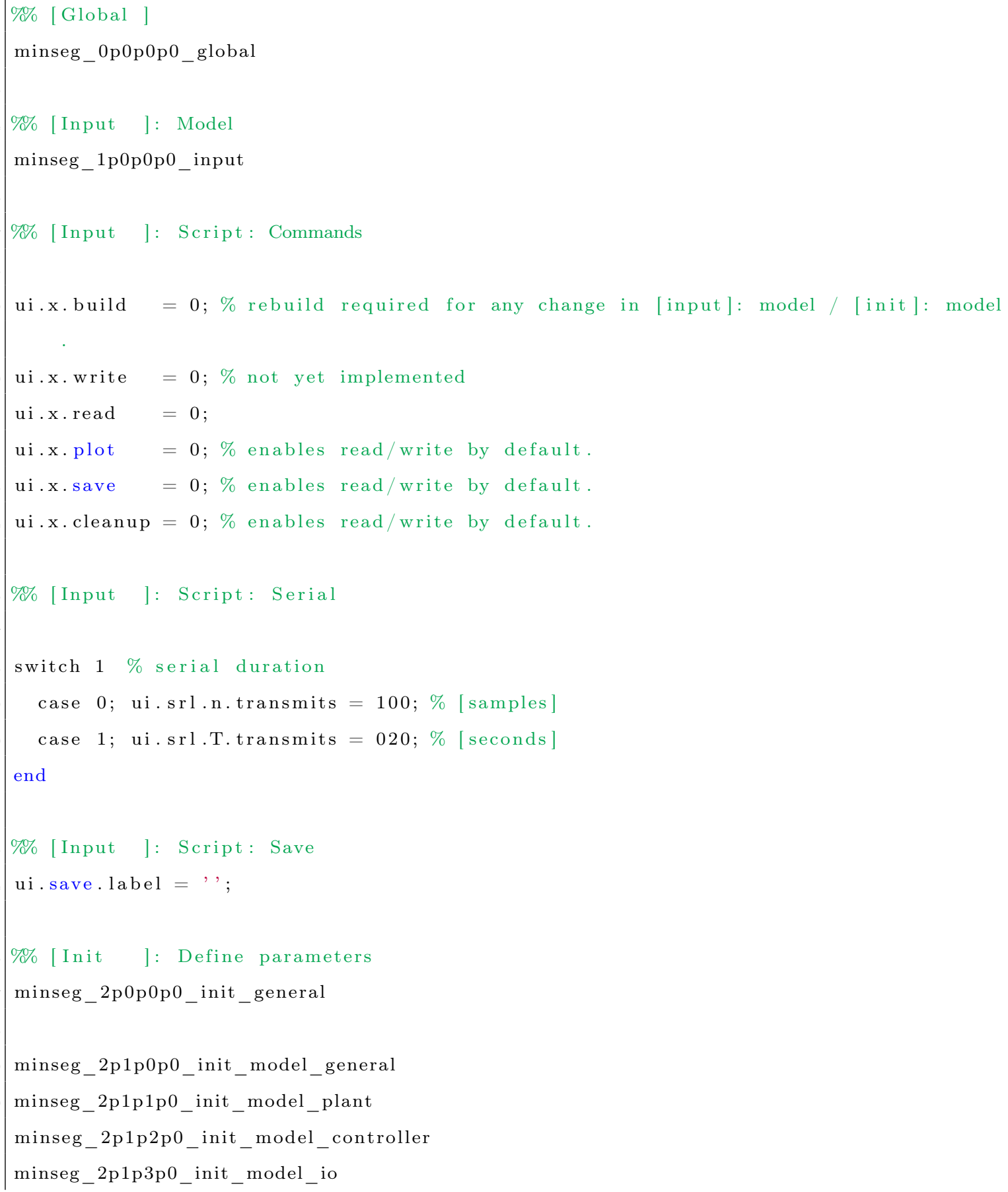




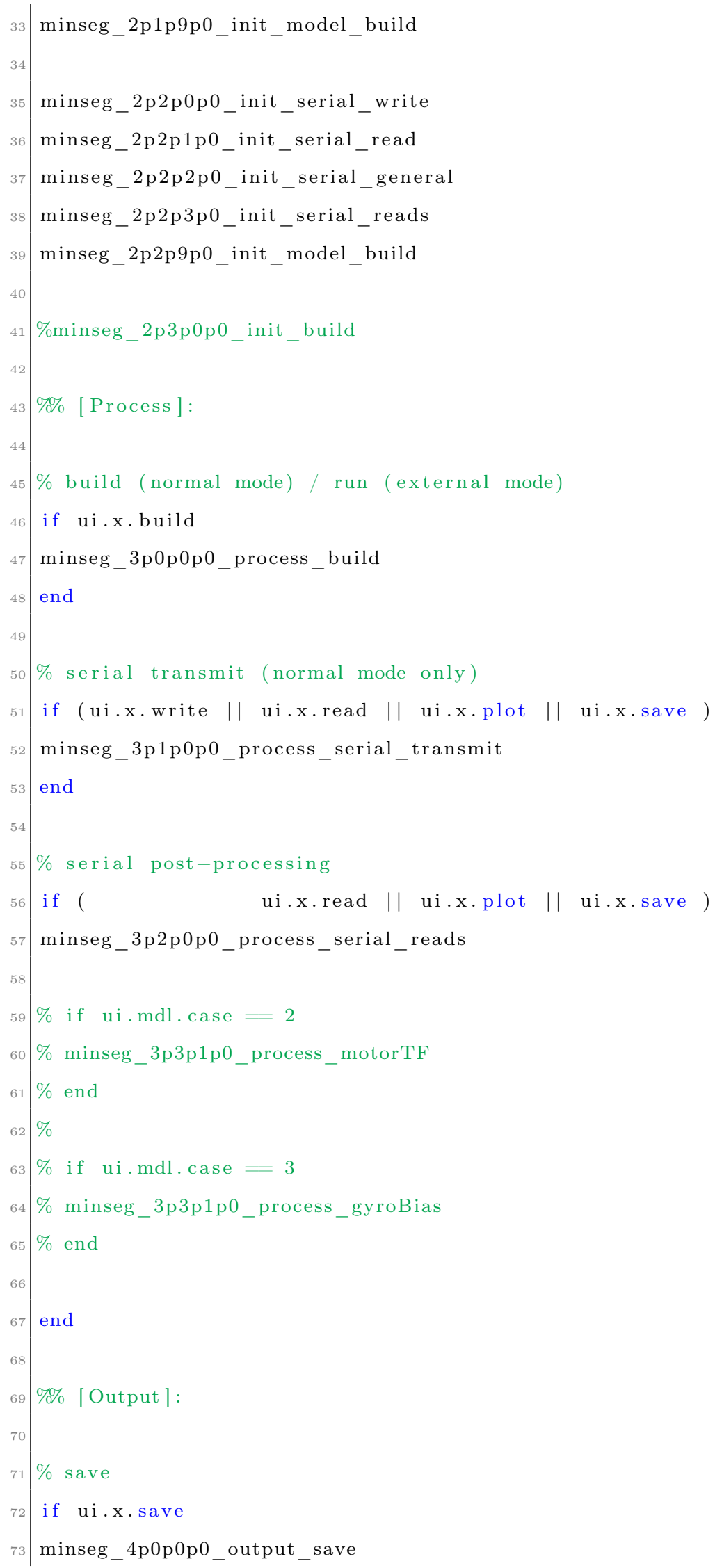




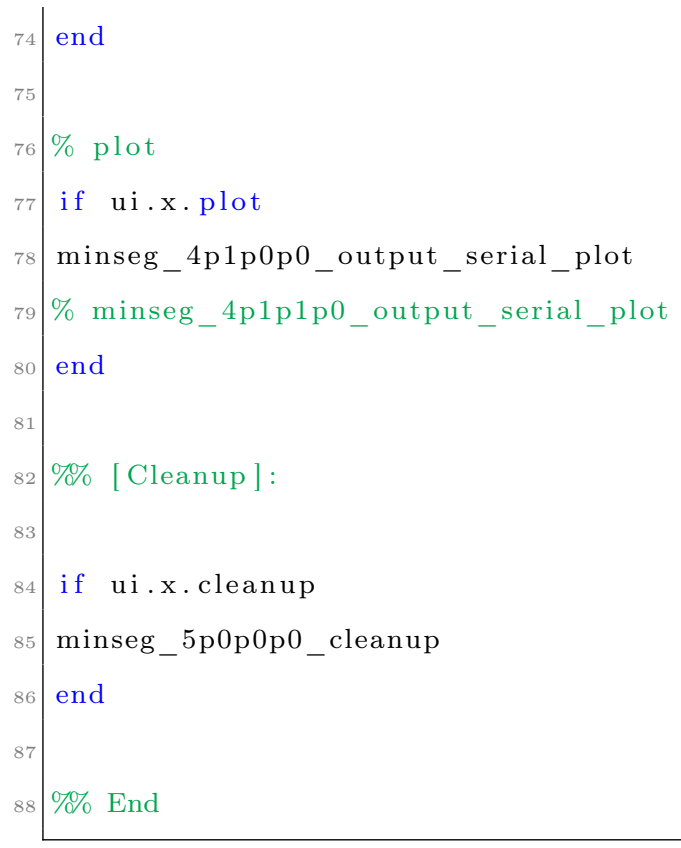

Code Listing A.1: [minseg.m]: Root file 
A.1.1. Global Setup

Code Listing A.2: [minseg.m]: Global Setup

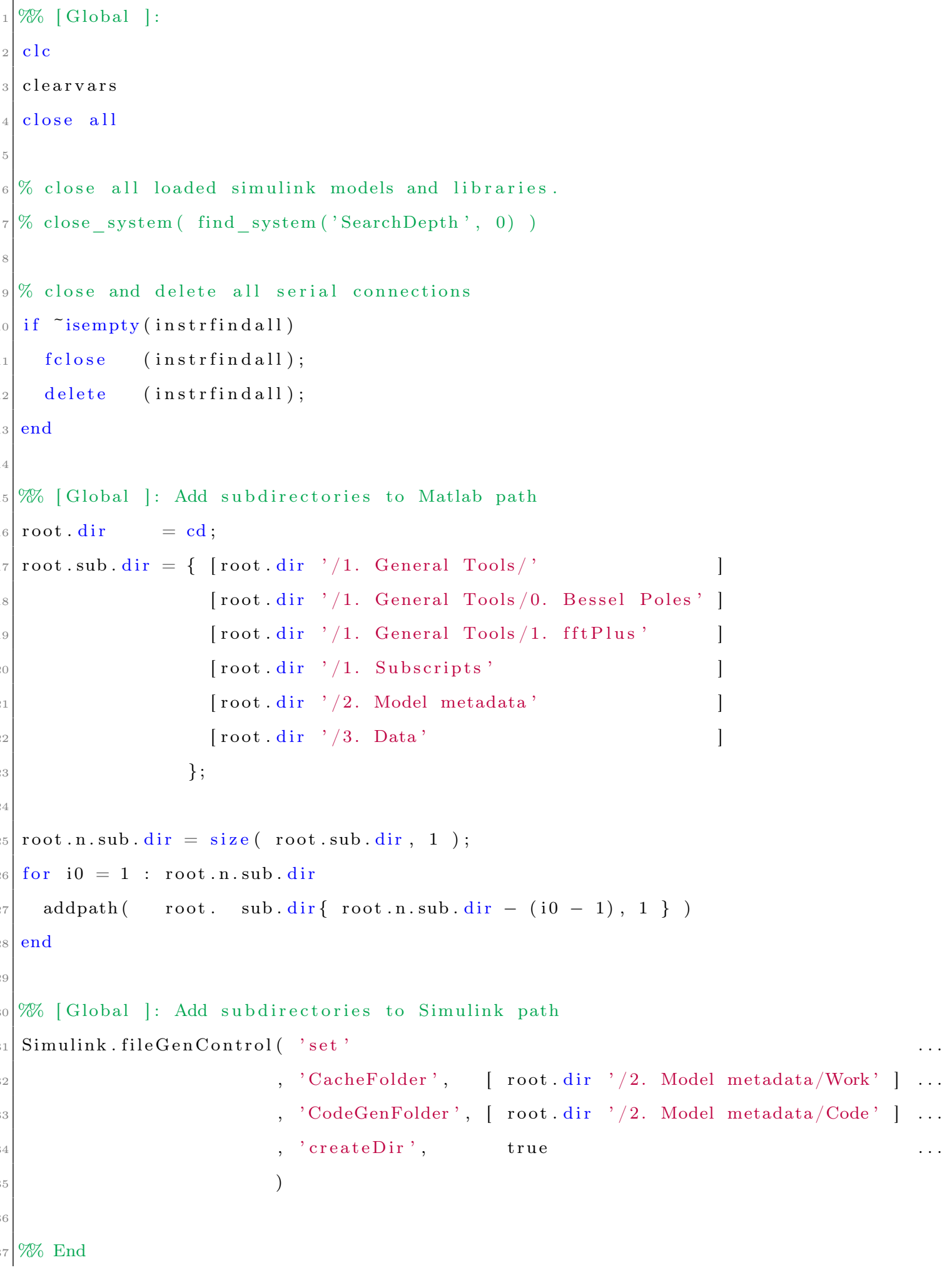


Code Listing A.2: [minseg.m]: Global Setup 


\section{A.1.2. User Inputs}

Code Listing A.3: [minseg.m]: User Inputs

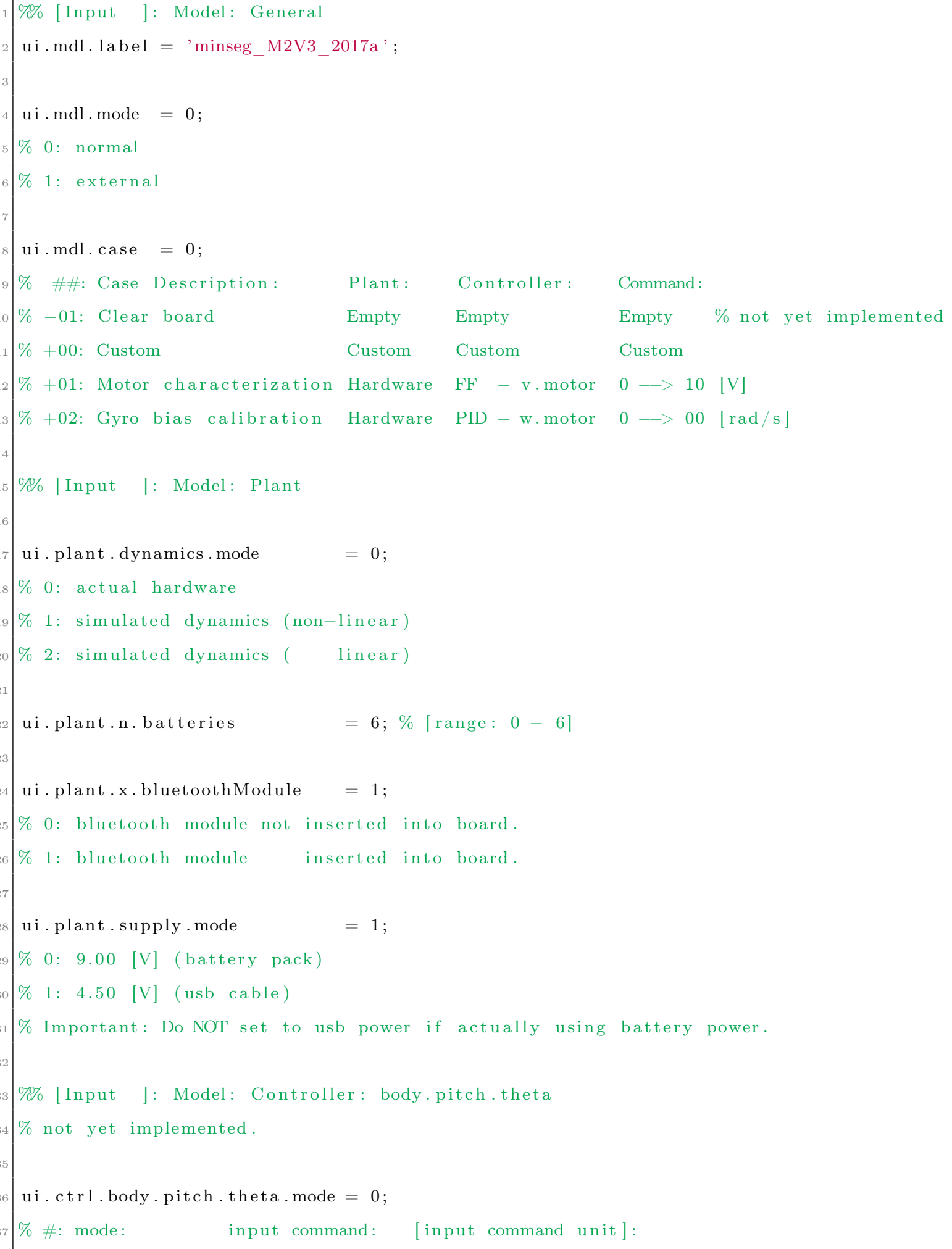




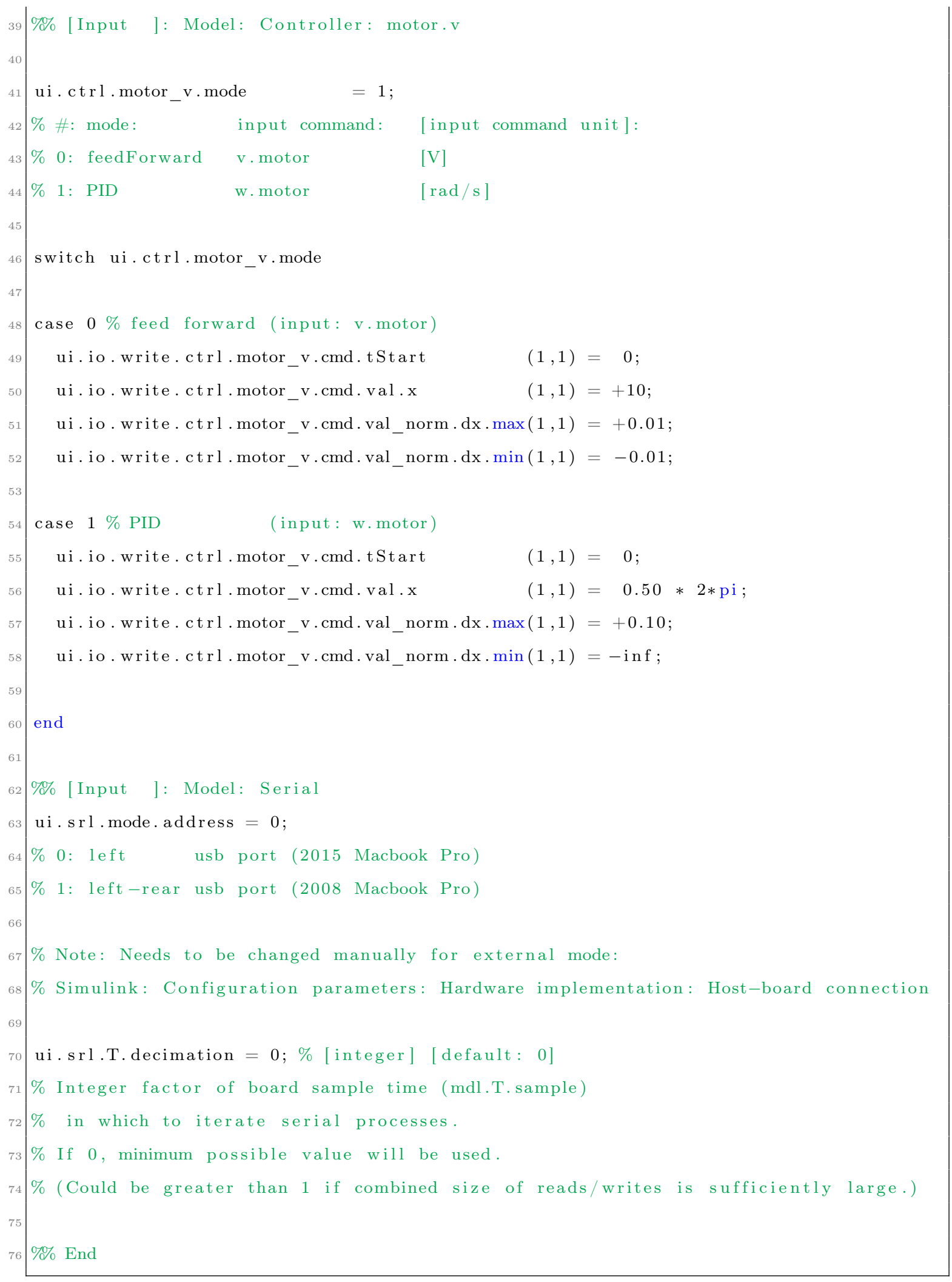

Code Listing A.3: [minseg.m]: User Inputs 
A.1.3. Initialization 
A.1.3.1. General

Code Listing A.4: [minseg.m]: Initialization - General

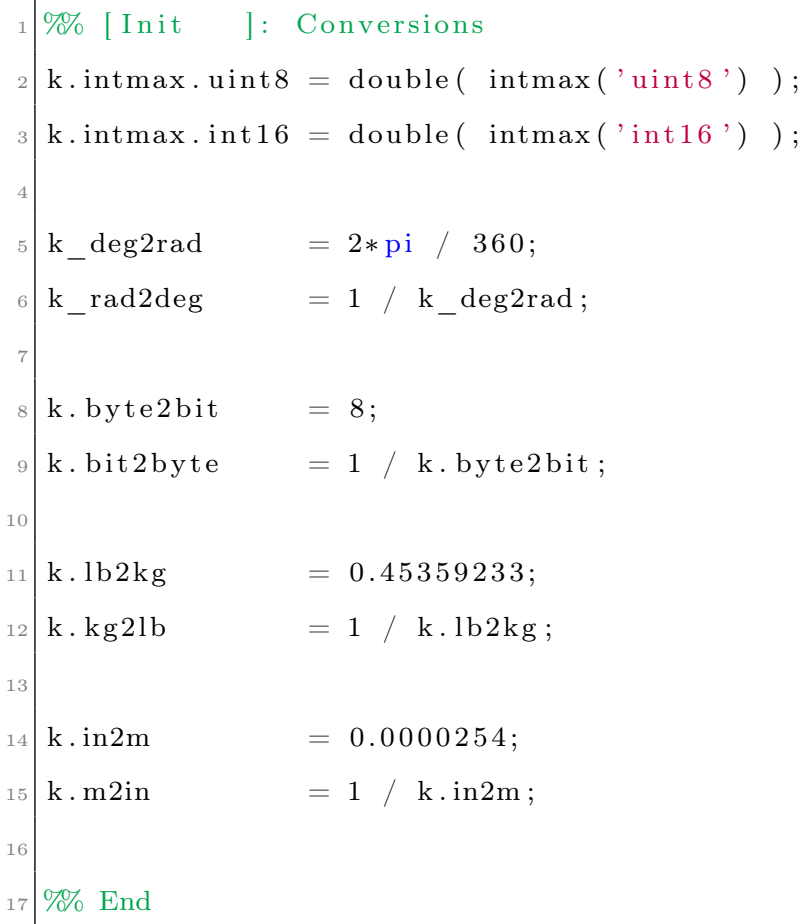

Code Listing A.4: [minseg.m]: Initialization - General 
A.1.3.2. Model 
A.1.3.2.1. General

Code Listing A.5: [minseg.m]: Initialization - Model - General

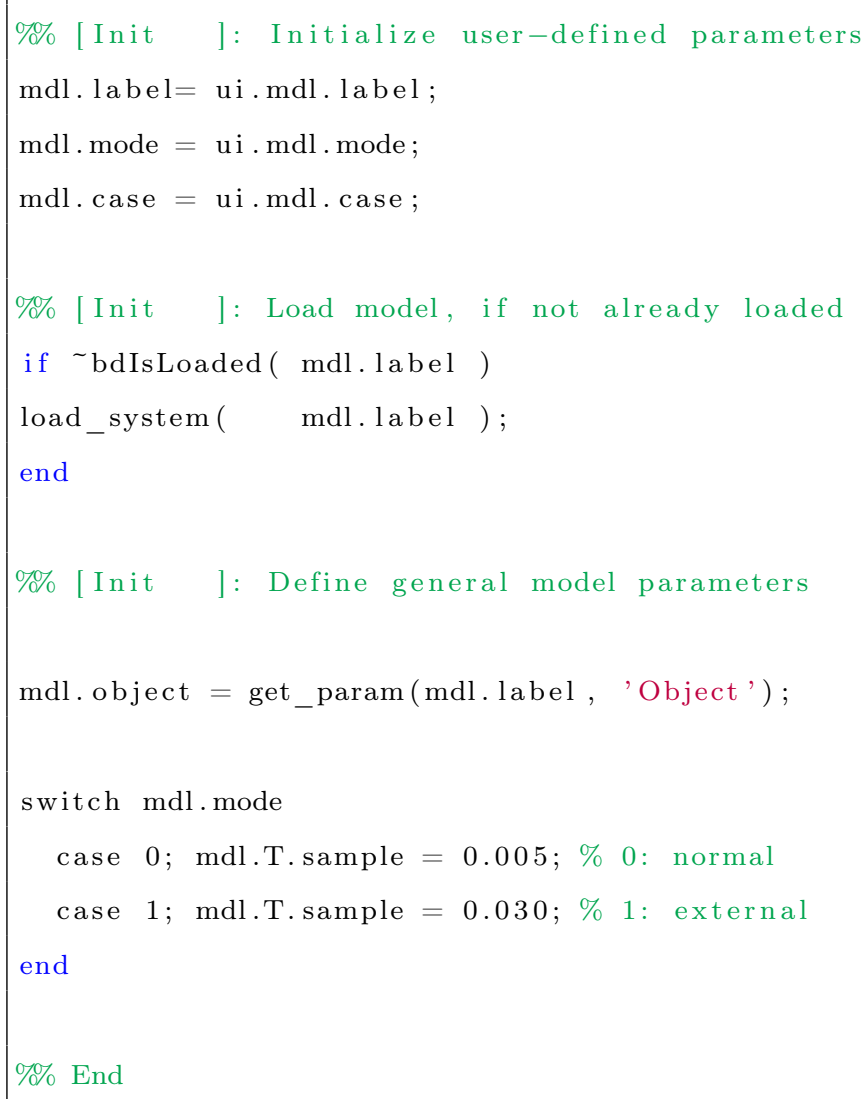

Code Listing A.5: [minseg.m]: Initialization - Model - General 


\section{A.1.3.2.2. Plant}

Code Listing A.6: [minseg.m]: Initialization - Model - Plant

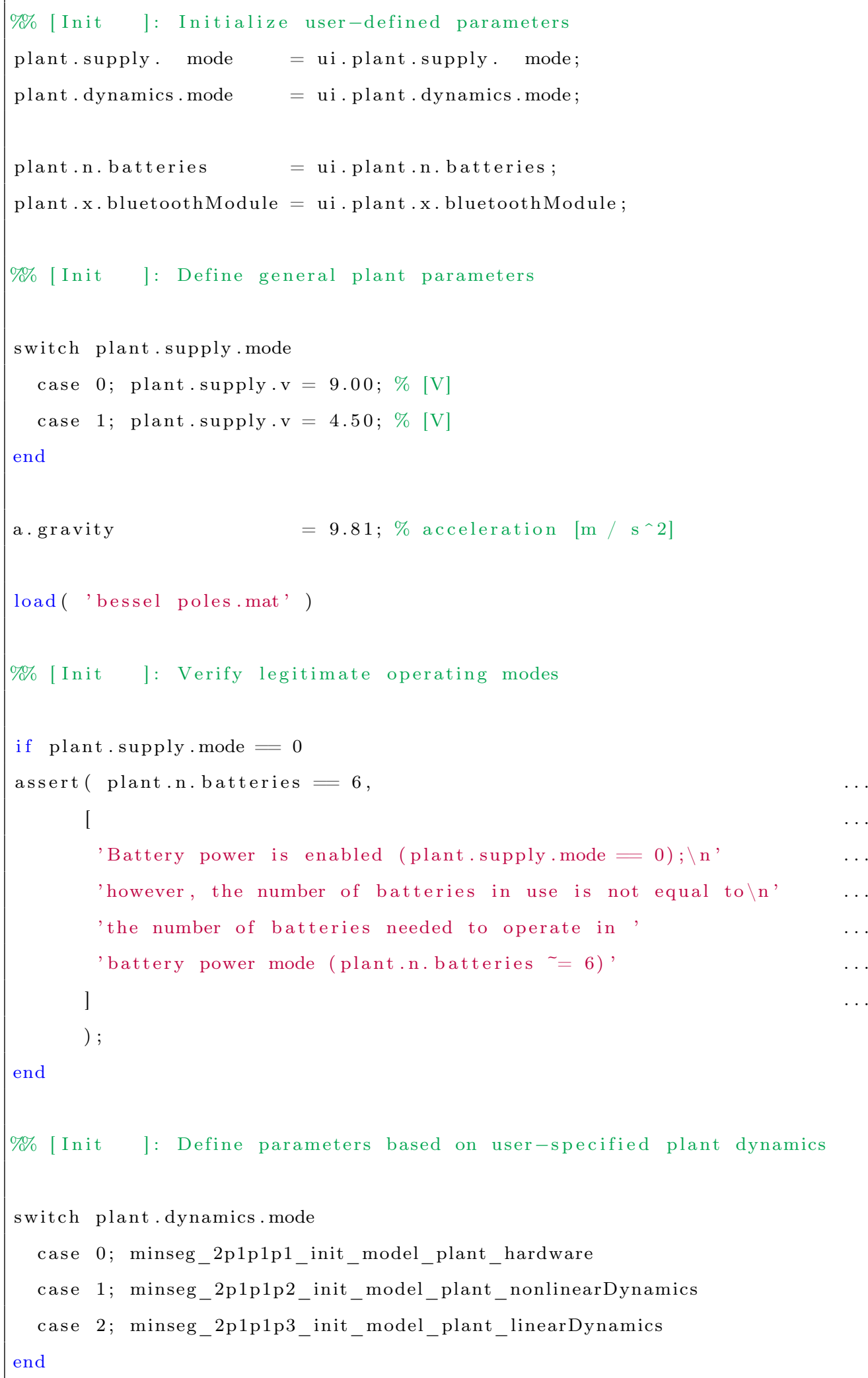


Code Listing A.6: [minseg.m]: Initialization - Model - Plant 
A.1.3.2.2.1. Hardware

Code Listing A.7: [minseg.m]: Initialization - Model - Plant - Hardware

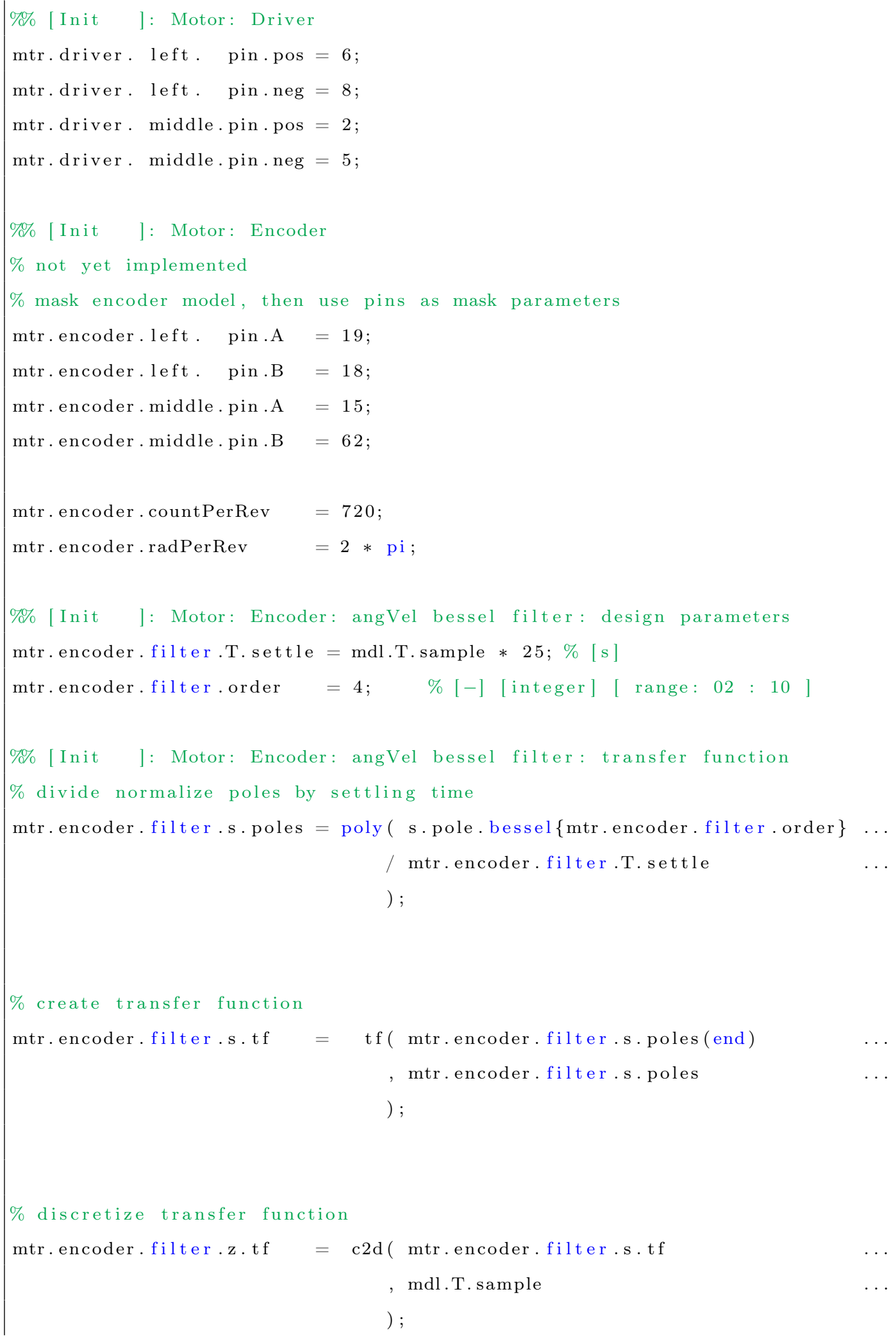




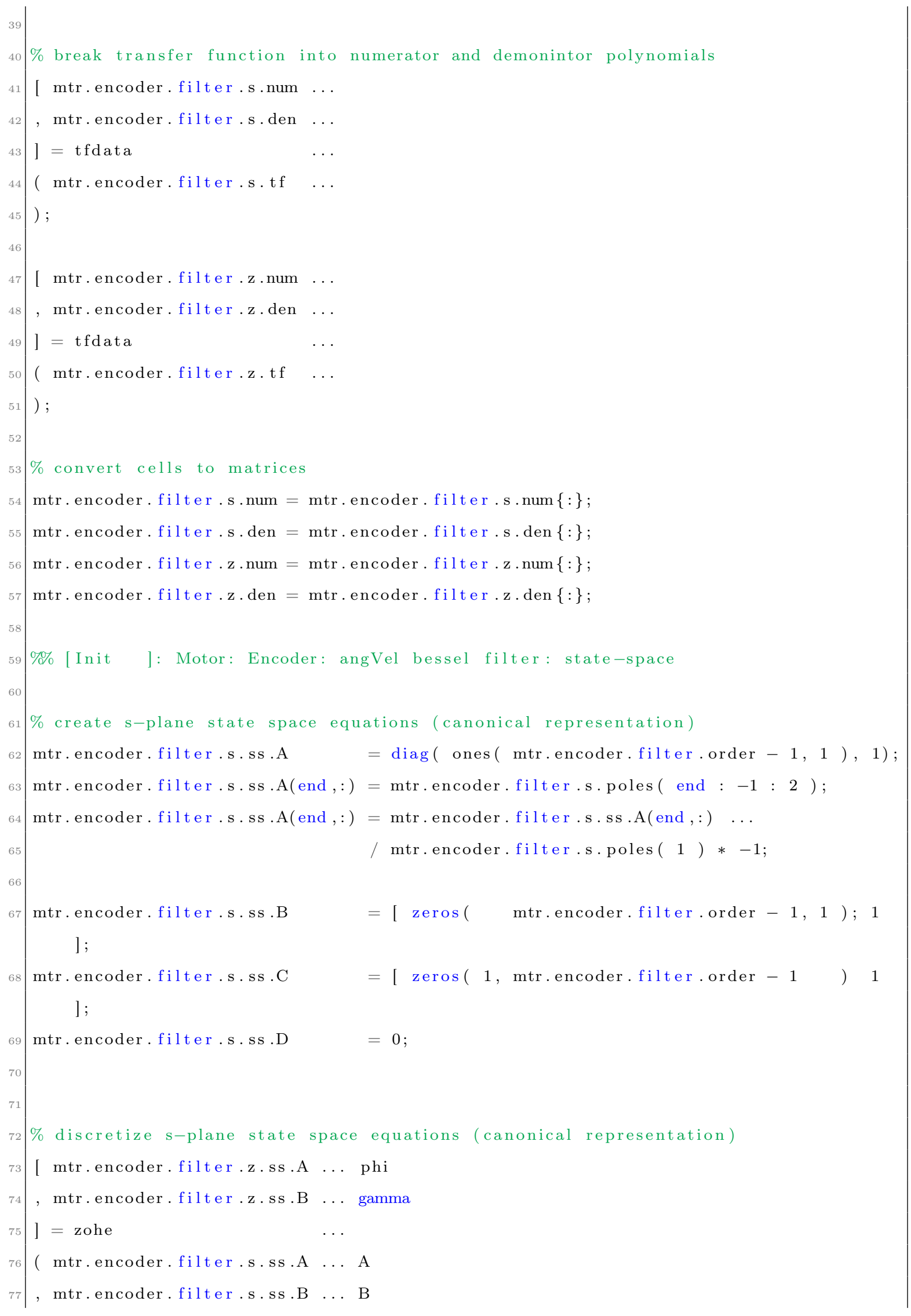




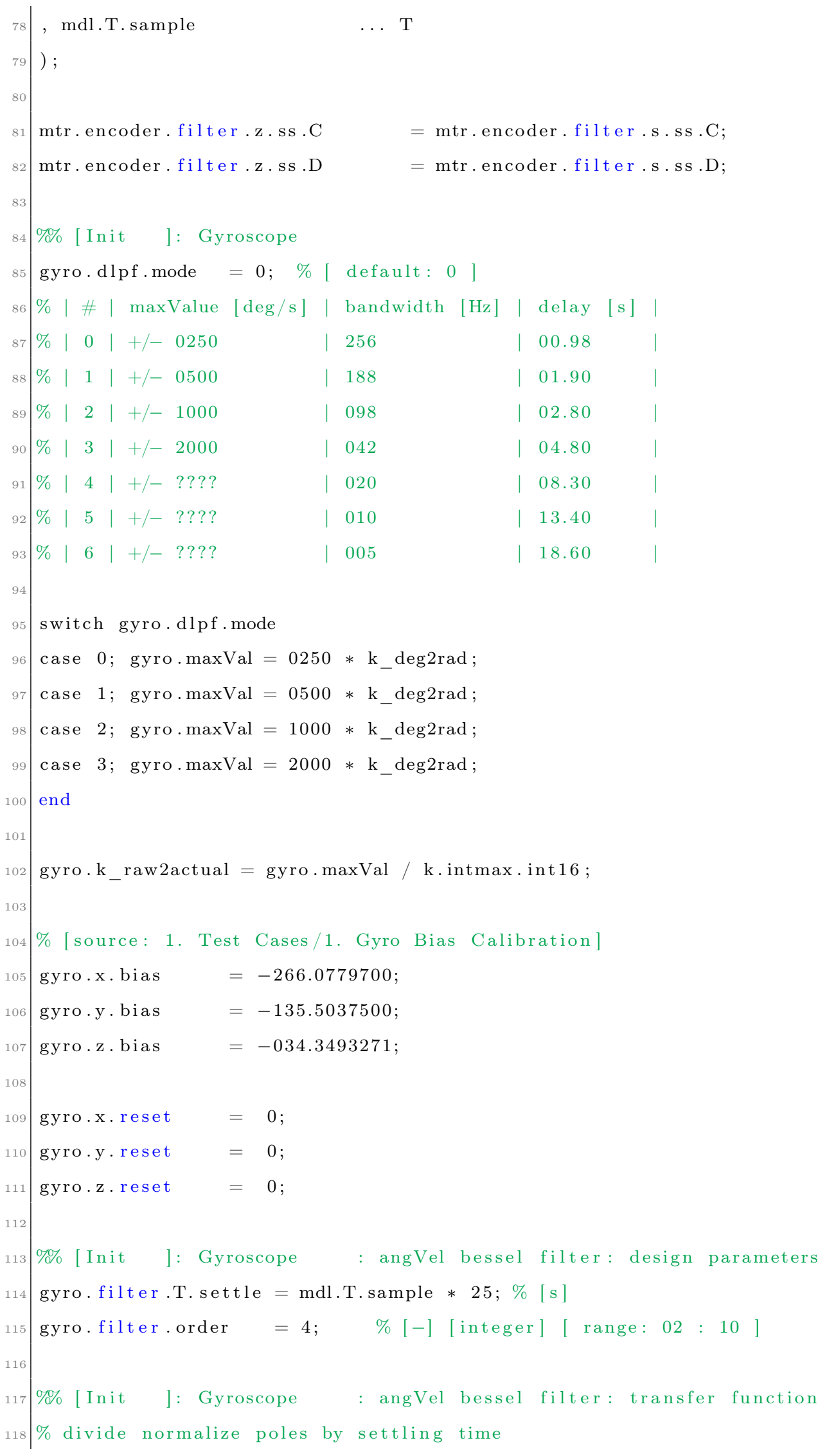




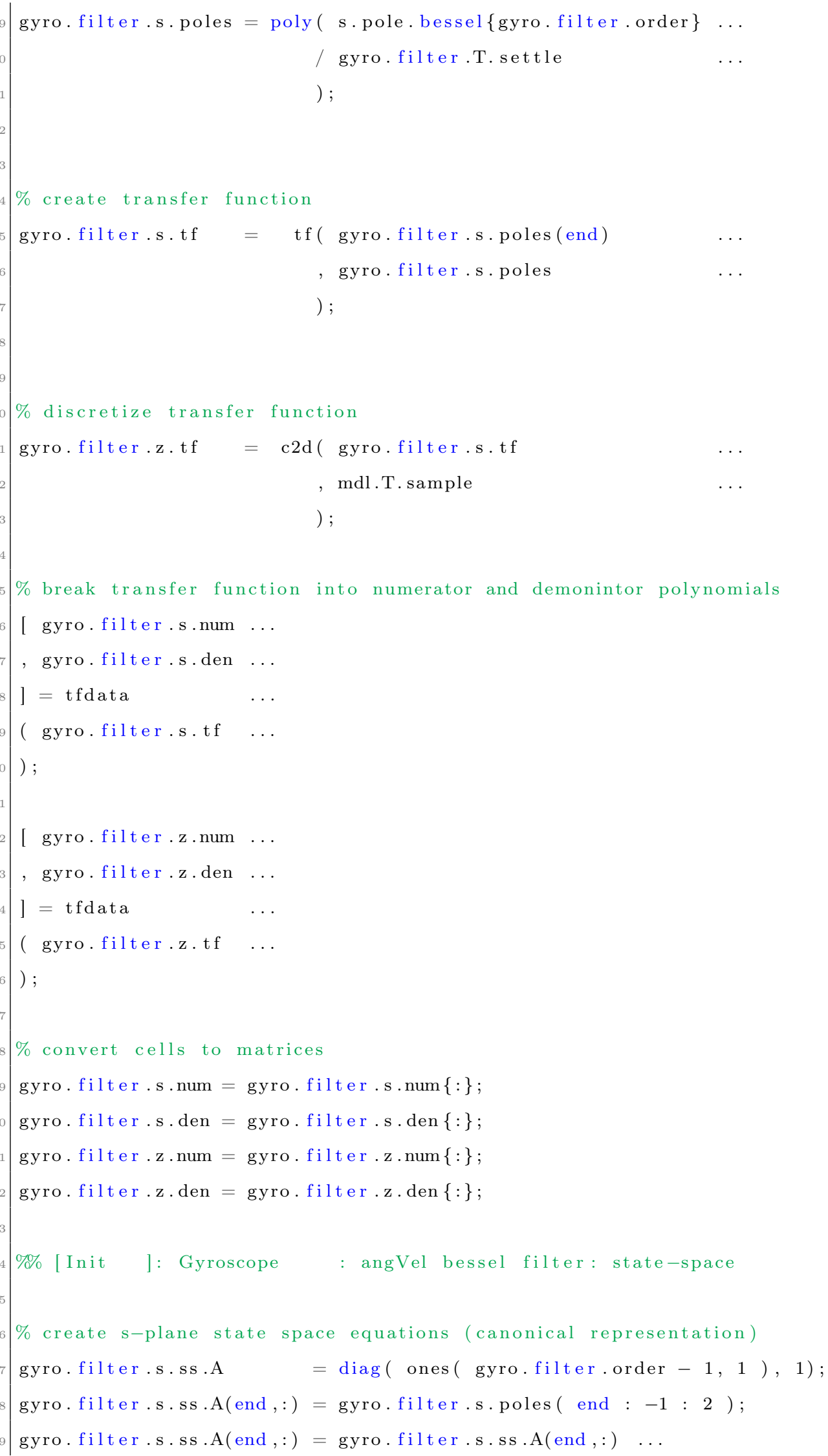




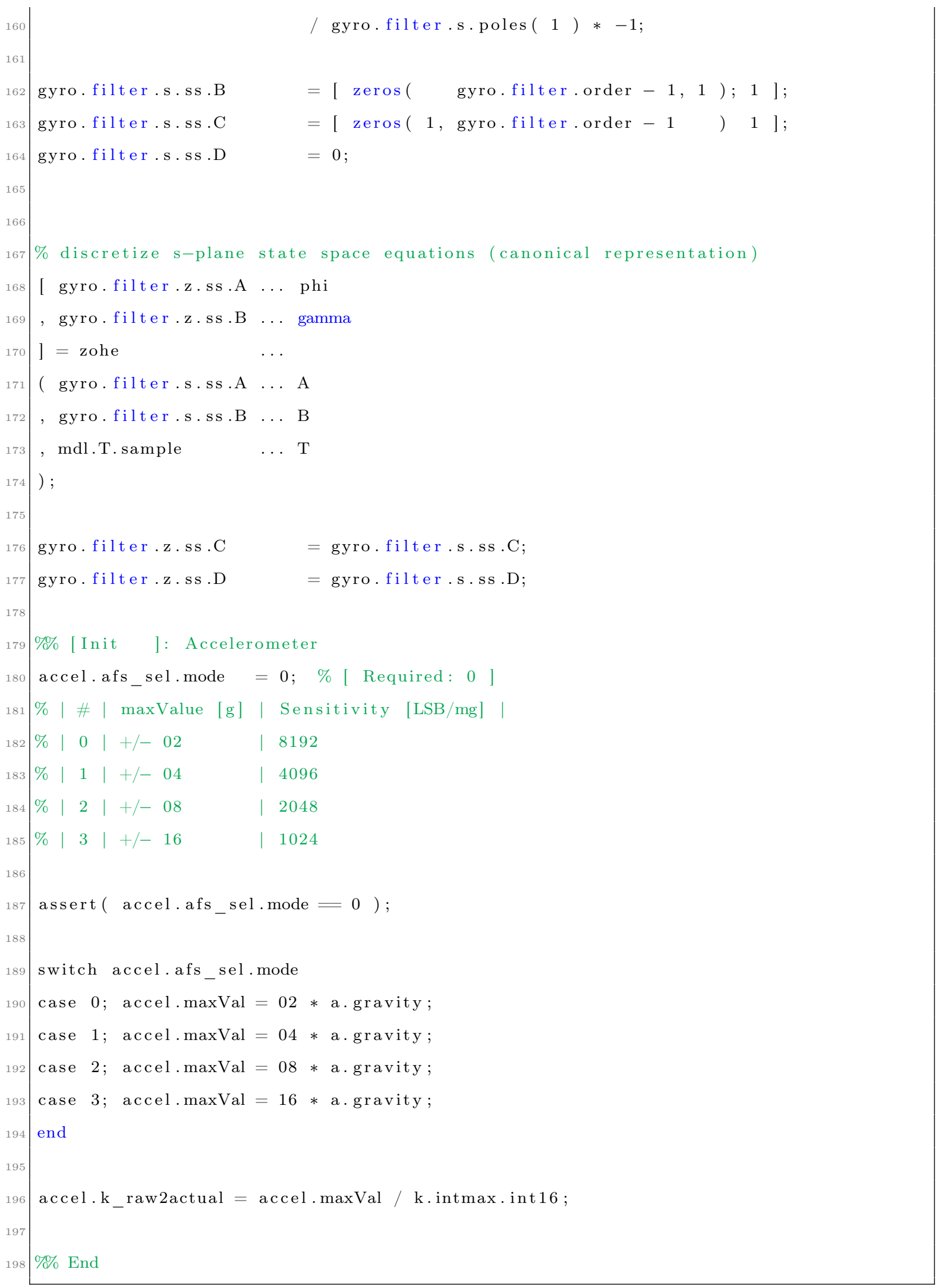

Code Listing A.7: [minseg.m]: Initialization - Model - Plant - Hardware 
A.1.3.2.2.2. Nonlinear Dynamics Model

Code Listing A.8: [minseg.m]: Initialization - Model - Plant - Nonlinear Dynamics Model $\% \%$ End

Code Listing A.8: [minseg.m]: Initialization - Model - Plant - Nonlinear Dynamics Model 


\section{A.1.3.2.2.3. Linear Dynamics Model}

Code Listing A.9: [minseg.m]: Initialization - Model - Plant - Linear Dynamics Model

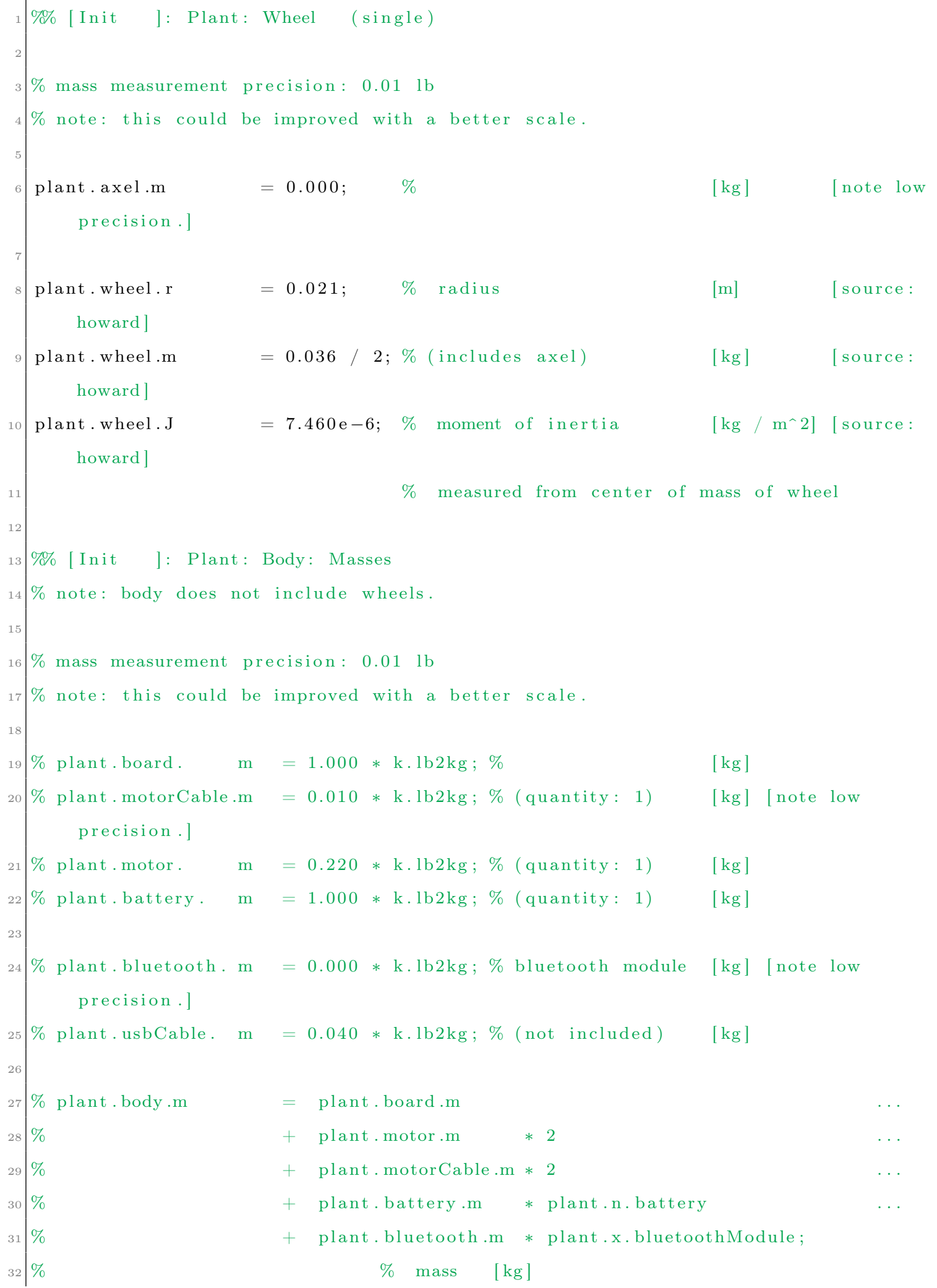




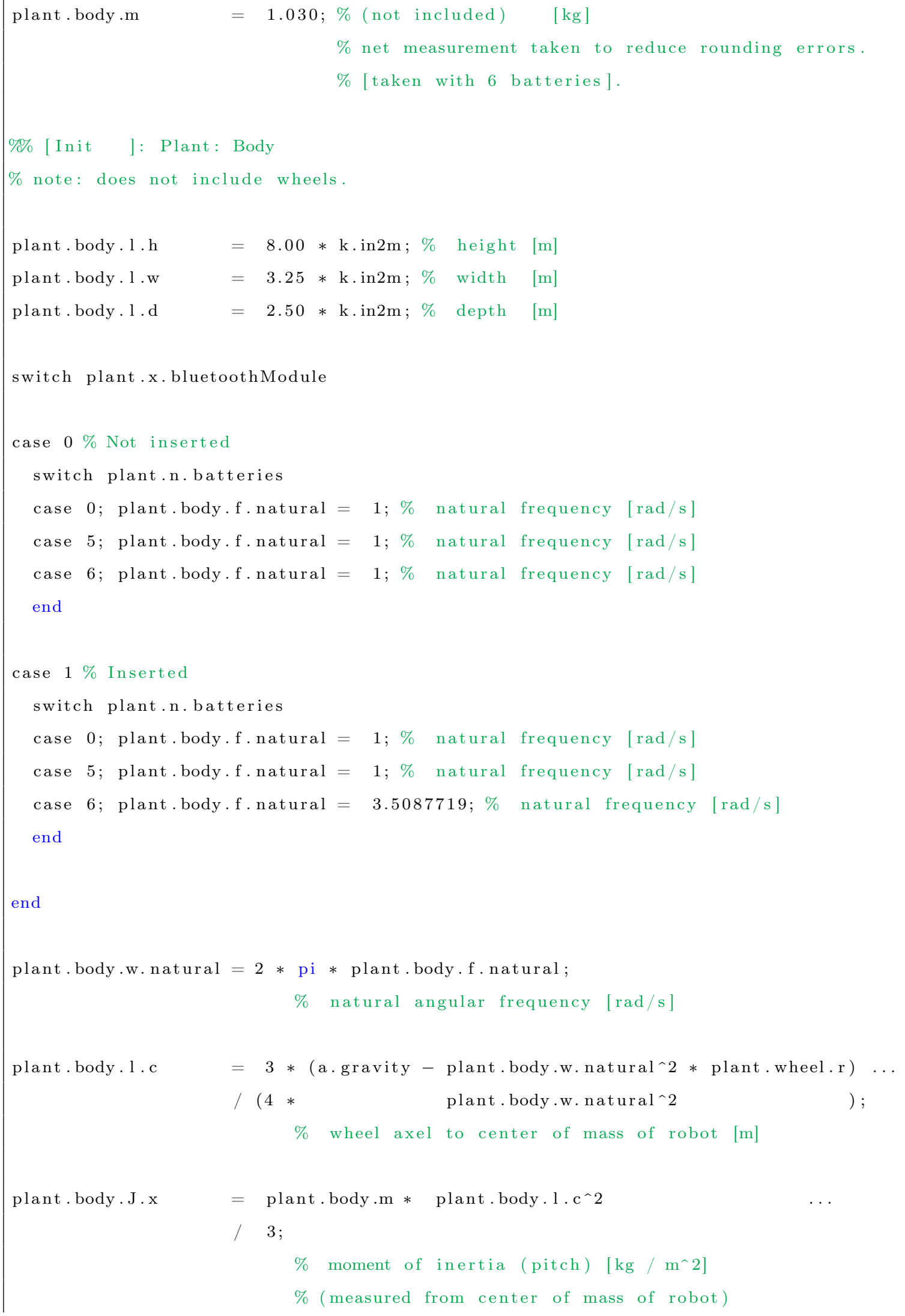




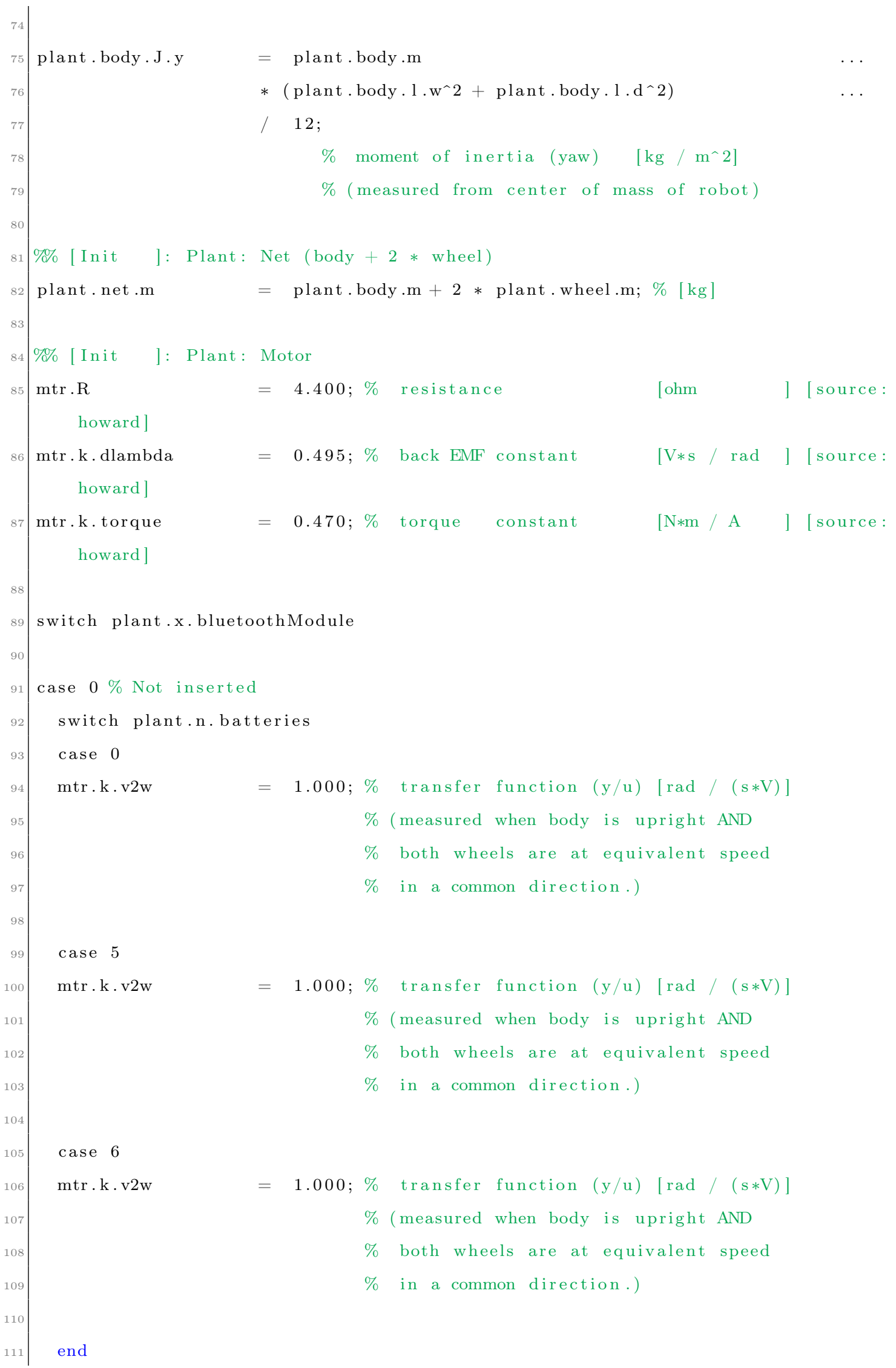




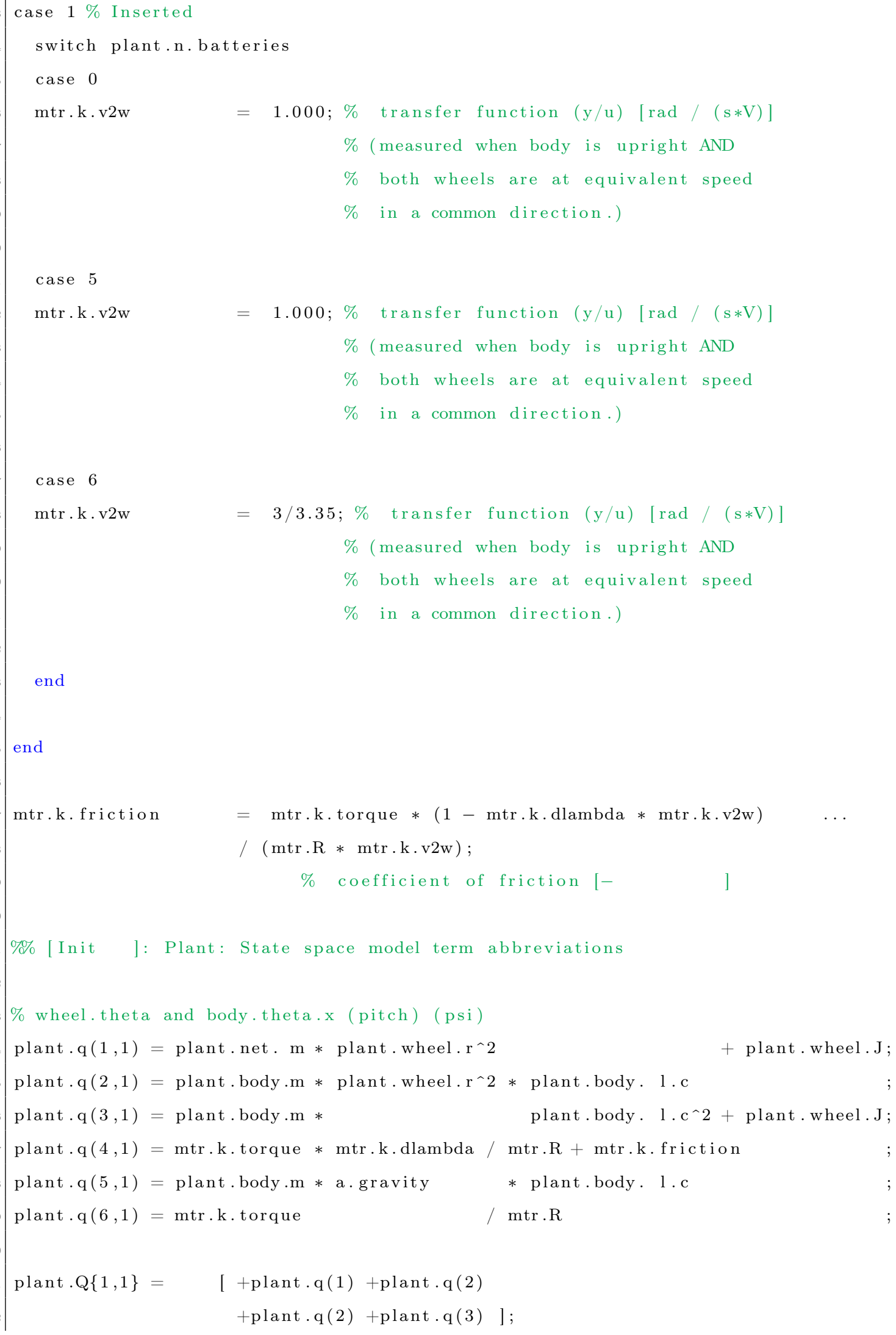




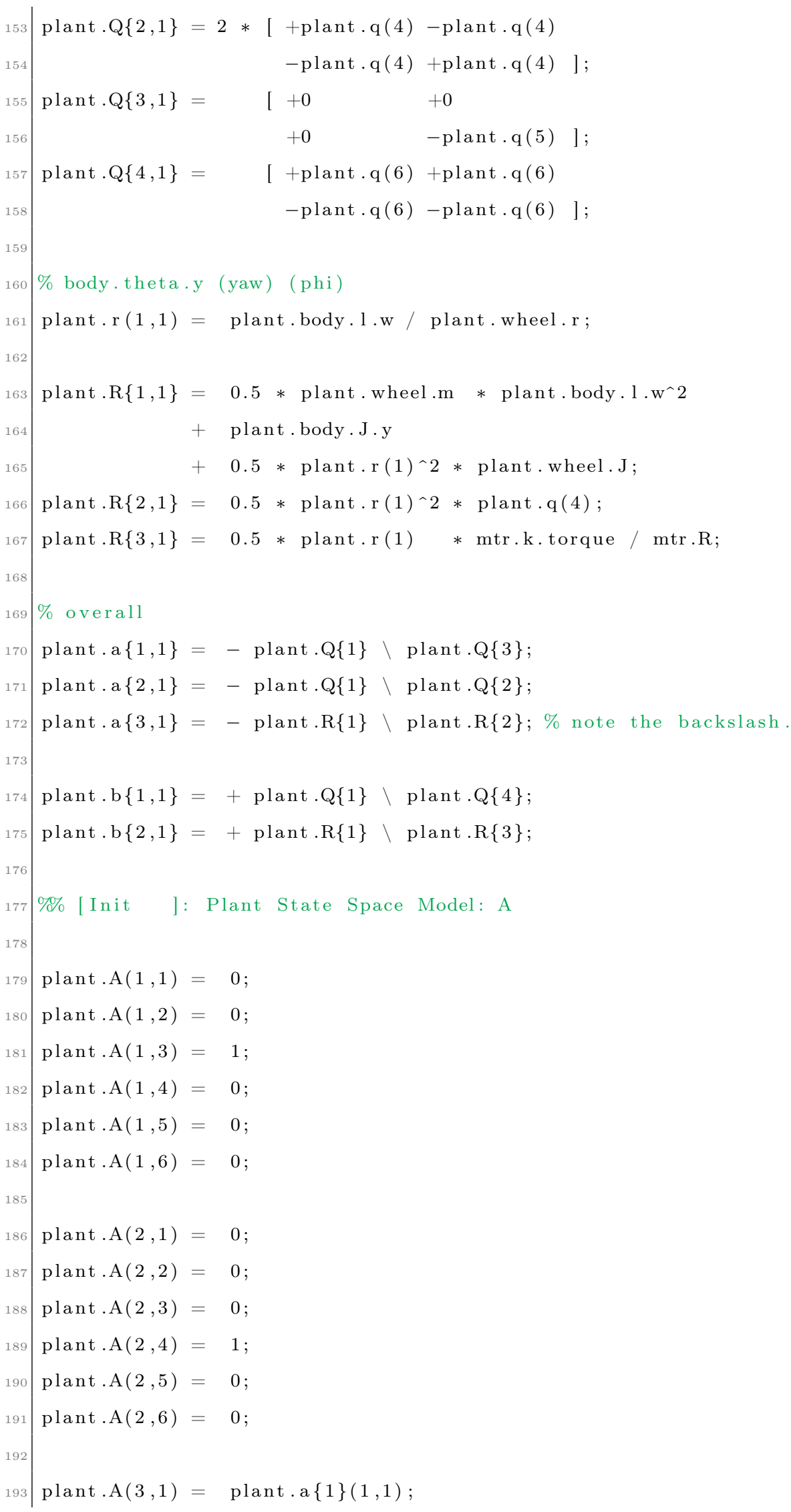




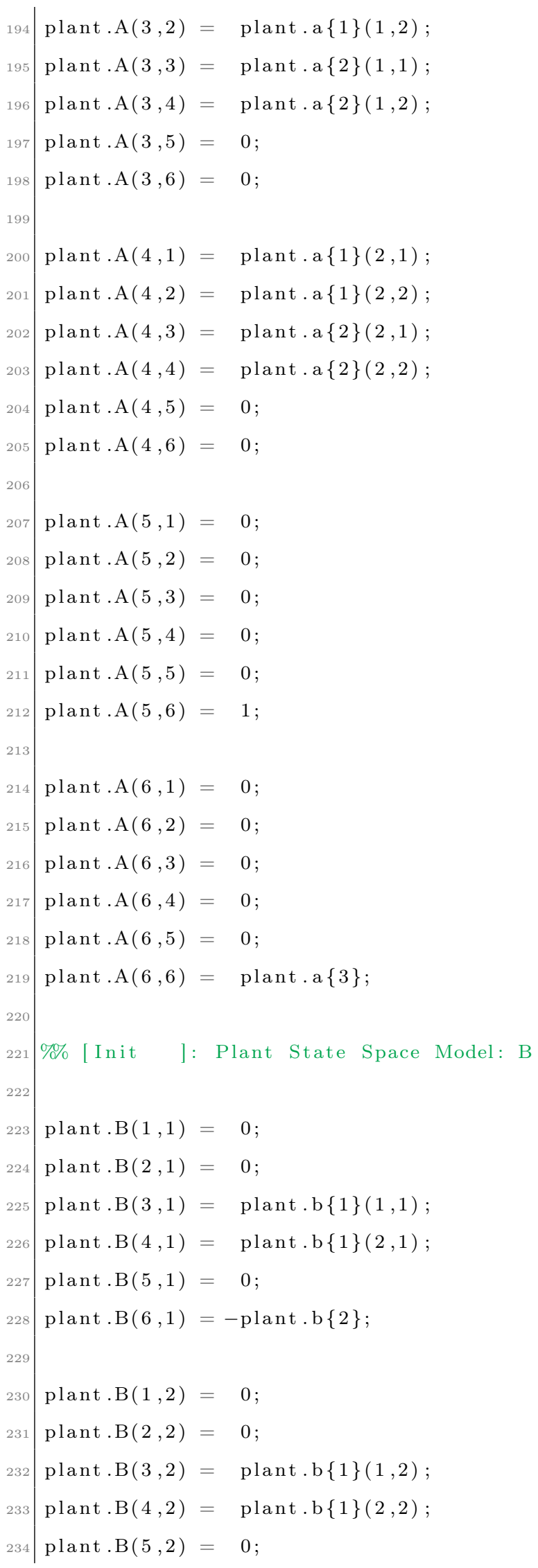




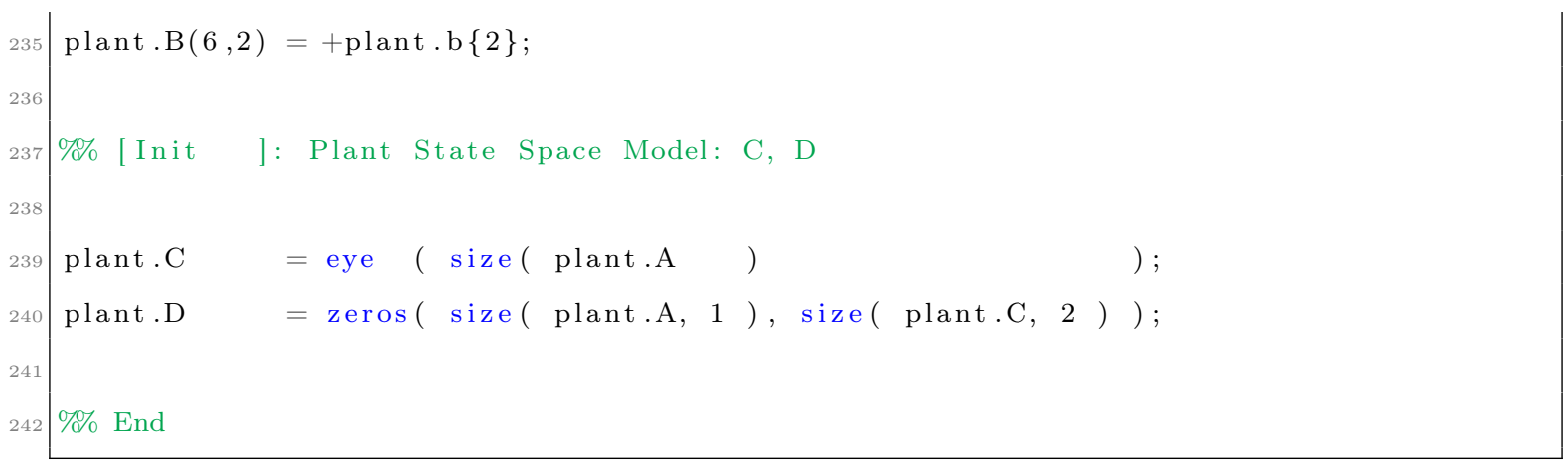

Code Listing A.9: [minseg.m]: Initialization - Model - Plant - Linear Dynamics Model 
A.1.3.2.3. Controller

Code Listing A.10: [minseg.m]: Initialization - Model - Controller

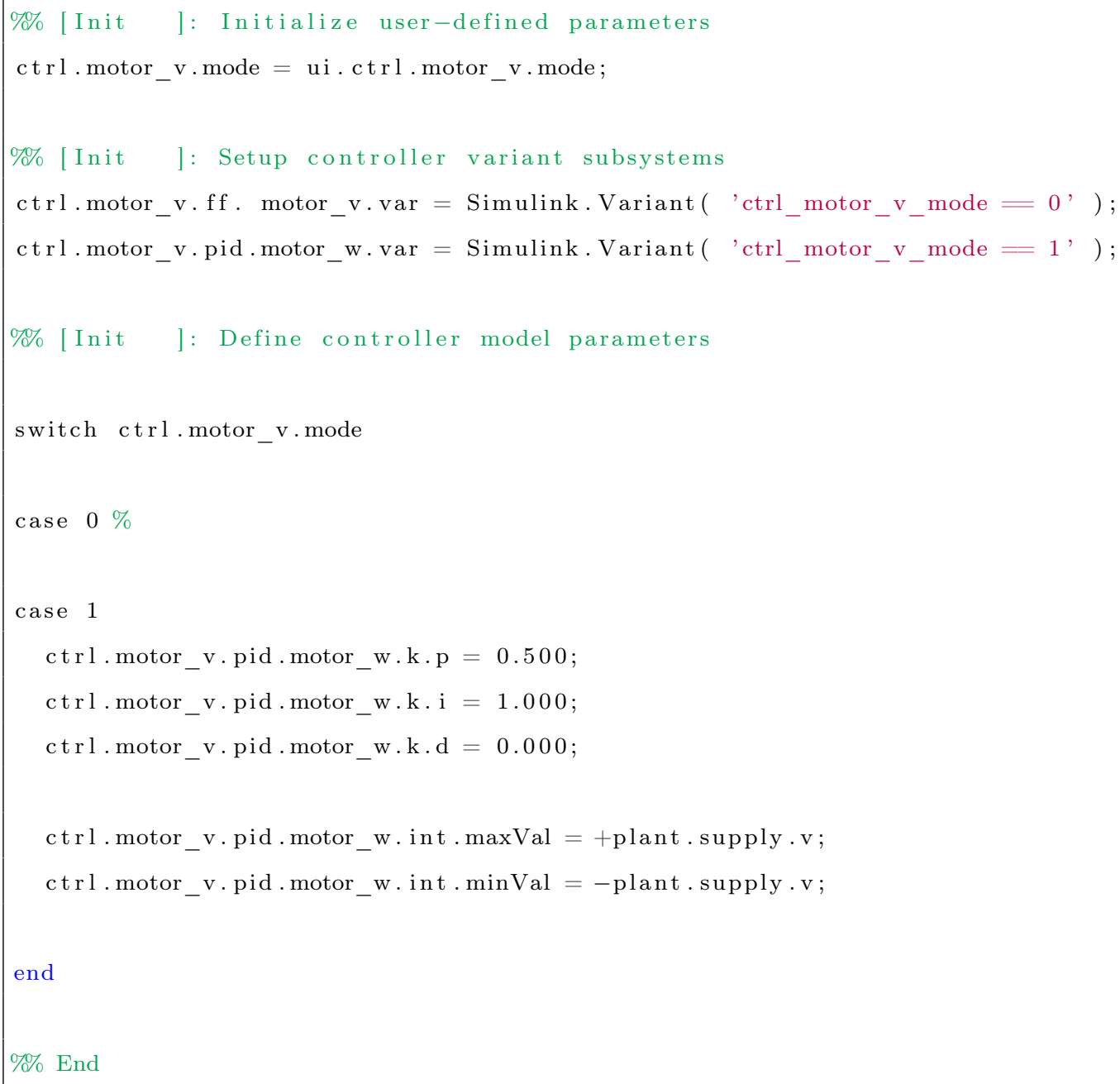

Code Listing A.10: [minseg.m]: Initialization - Model - Controller 


\section{A.1.3.2.4. Board Inputs and Outputs}

Code Listing A.11: [minseg.m]: Initialization - Model - User-Defined Board Inputs and Outputs

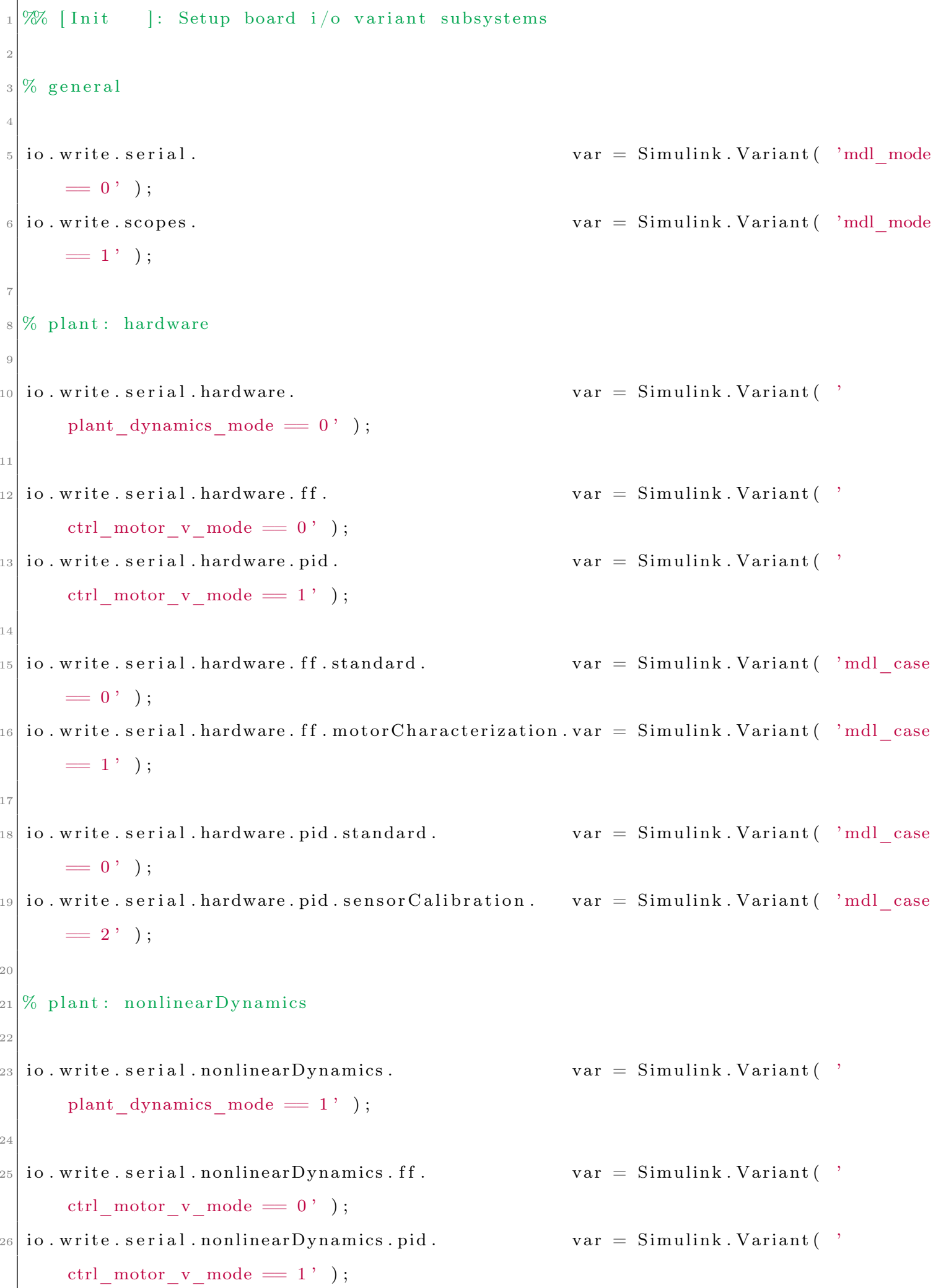




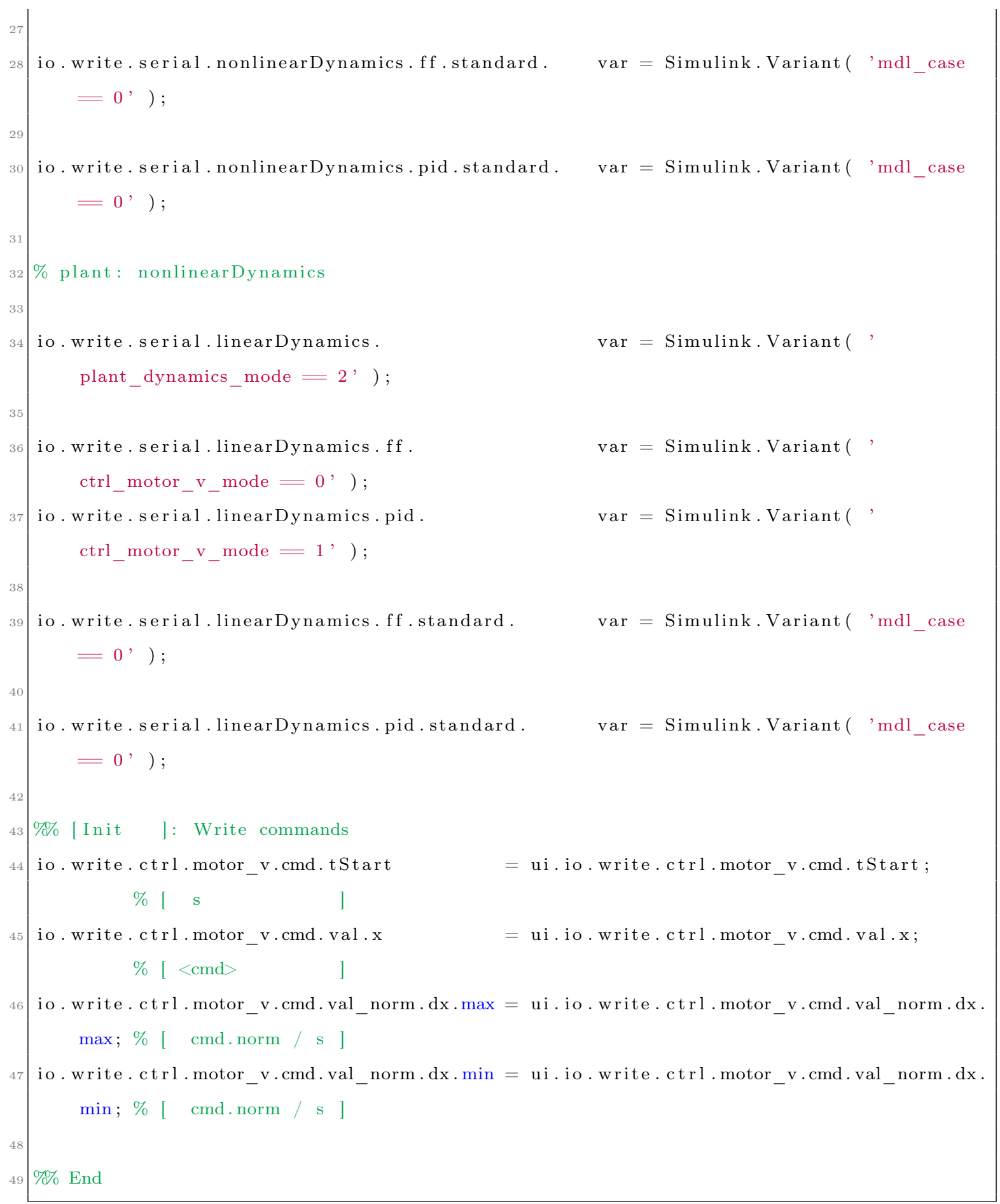

Code Listing A.11: [minseg.m]: Initialization - Model - User-Defined Board Inputs and Outputs 


\section{A.1.3.2.5. Build Parameters}

Code Listing A.12: [minseg.m]: Initialization - Model - Model Build Parameters

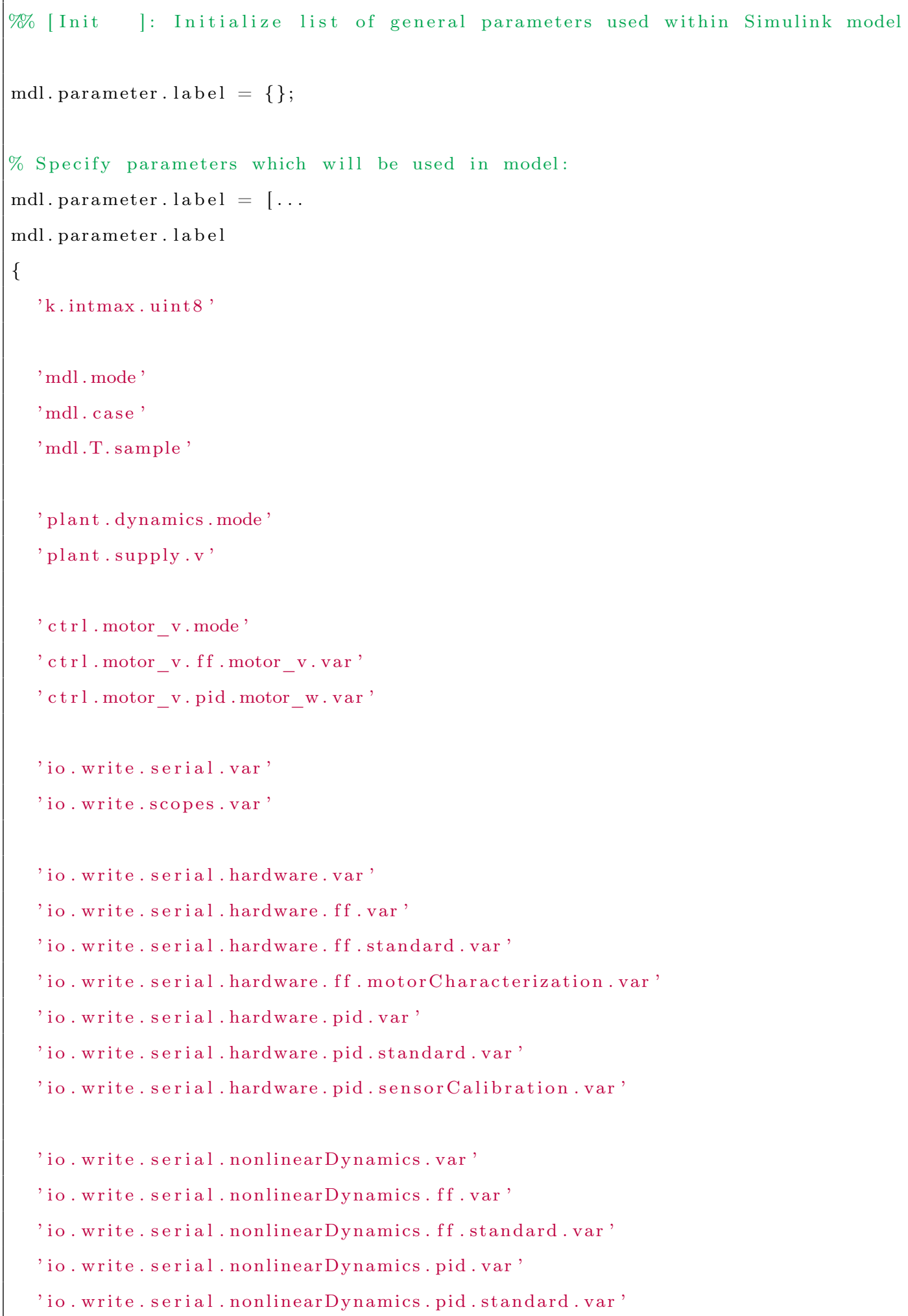




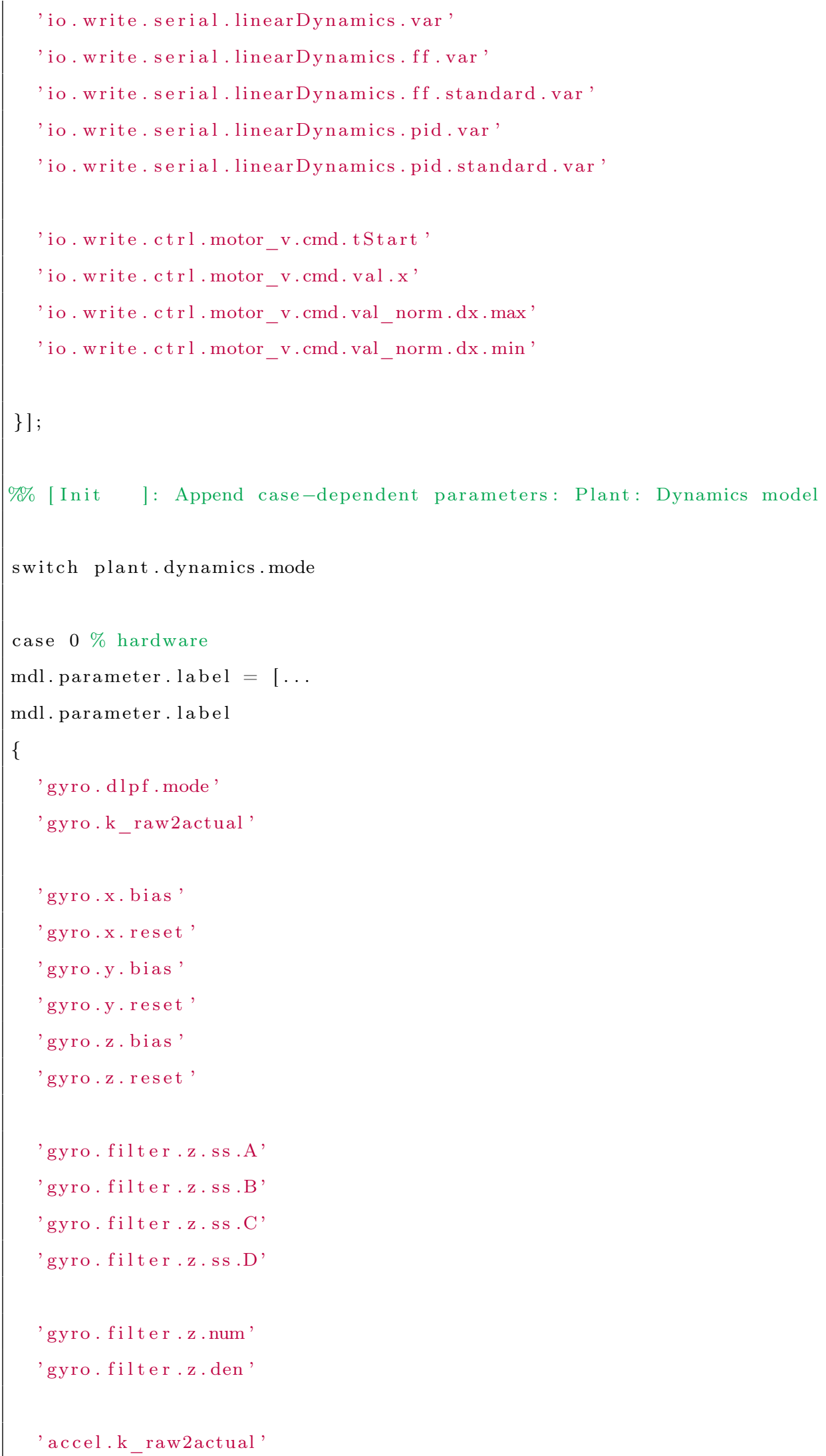




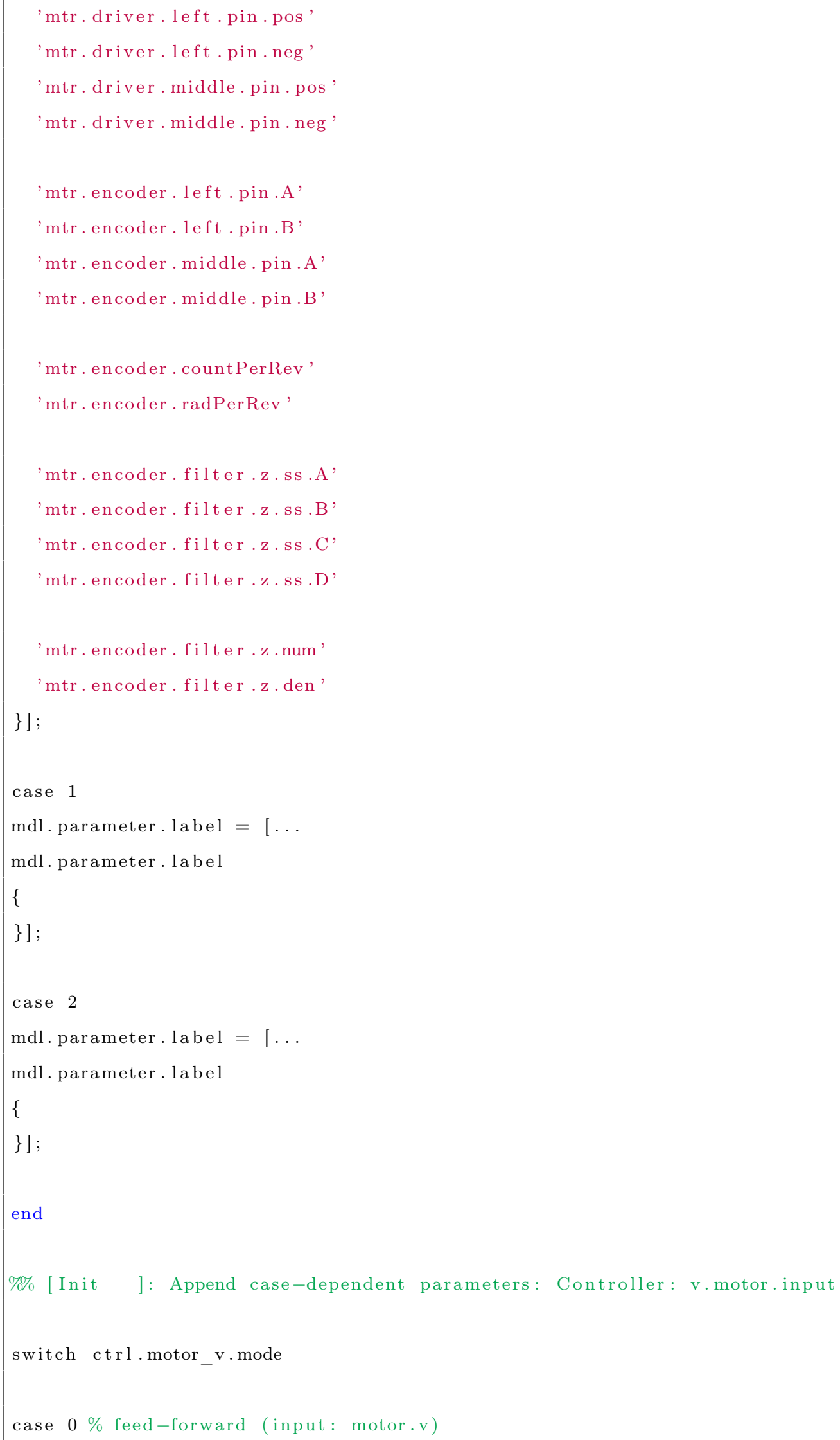




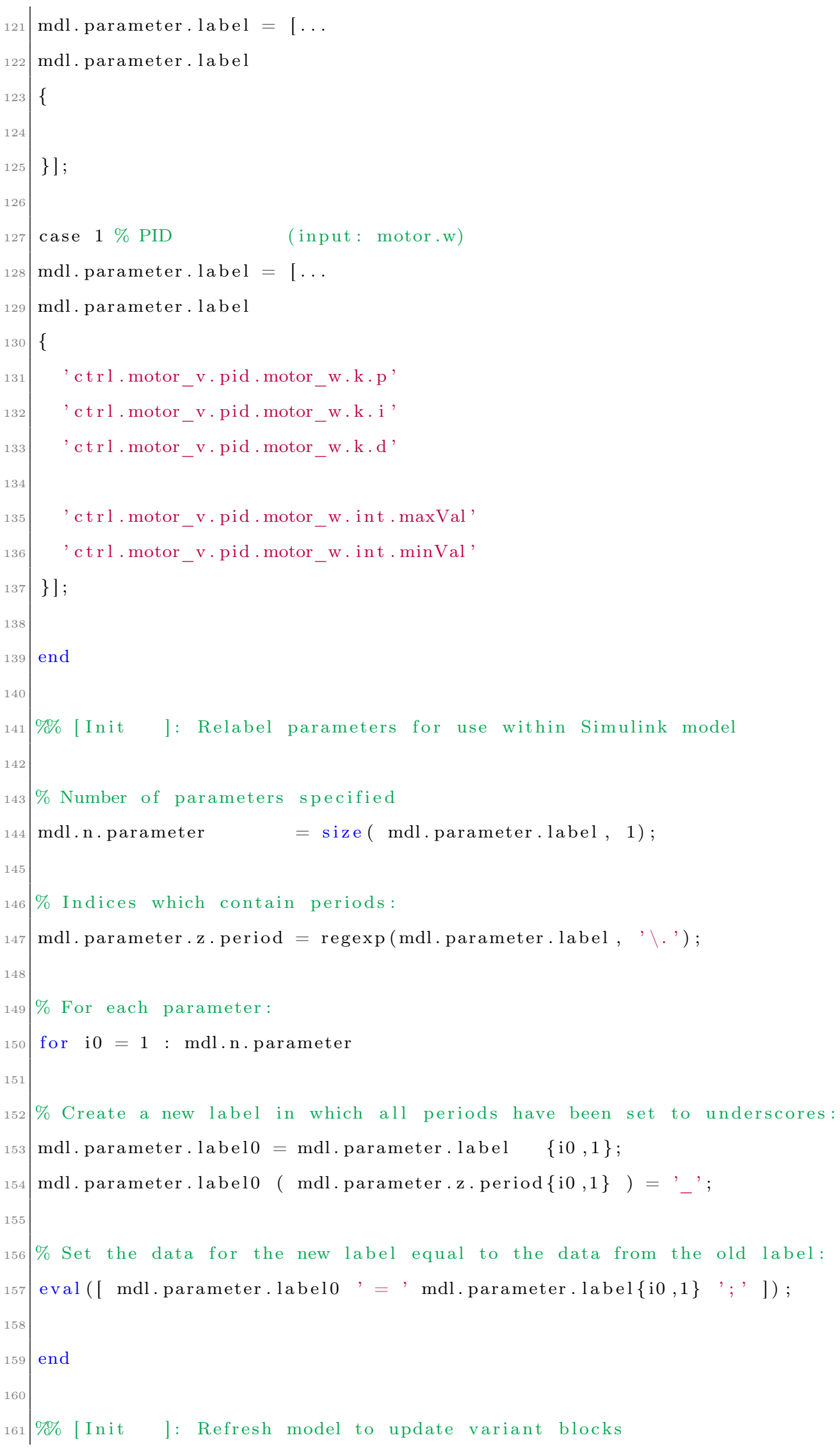




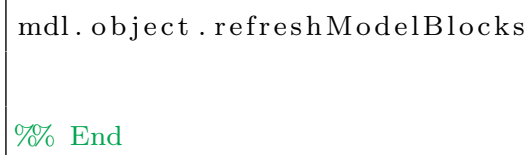

Code Listing A.12: [minseg.m]: Initialization - Model - Model Build Parameters 
A.1.3.3. Serial 
A.1.3.3.1. Write

Code Listing A.13: [minseg.m]: Initialization - Serial - Write

Code Listing A.13: [minseg.m]: Initialization - Serial - Write 
A.1.3.3.2. Read

Code Listing A.14: [minseg.m]: Initialization - Serial - Read

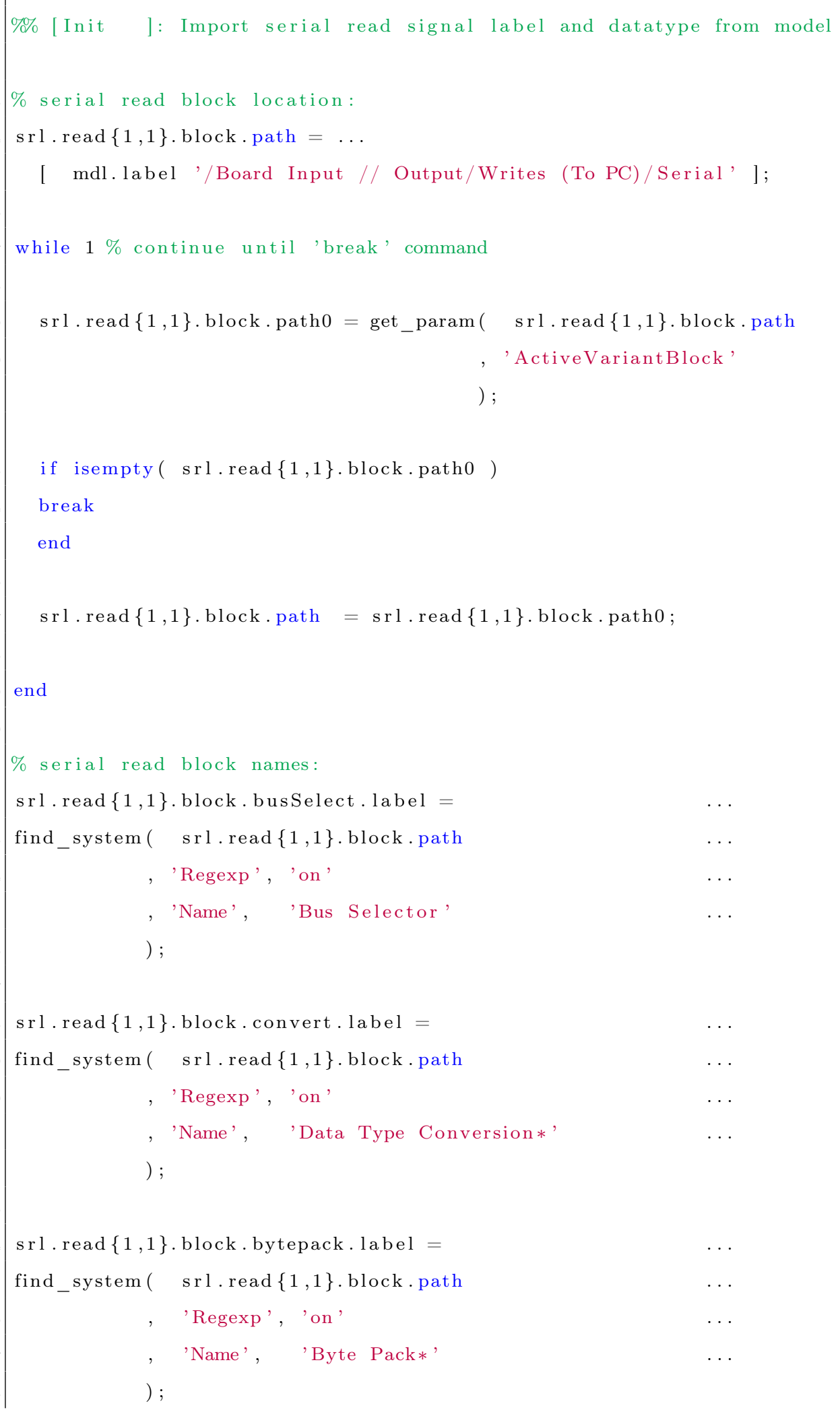




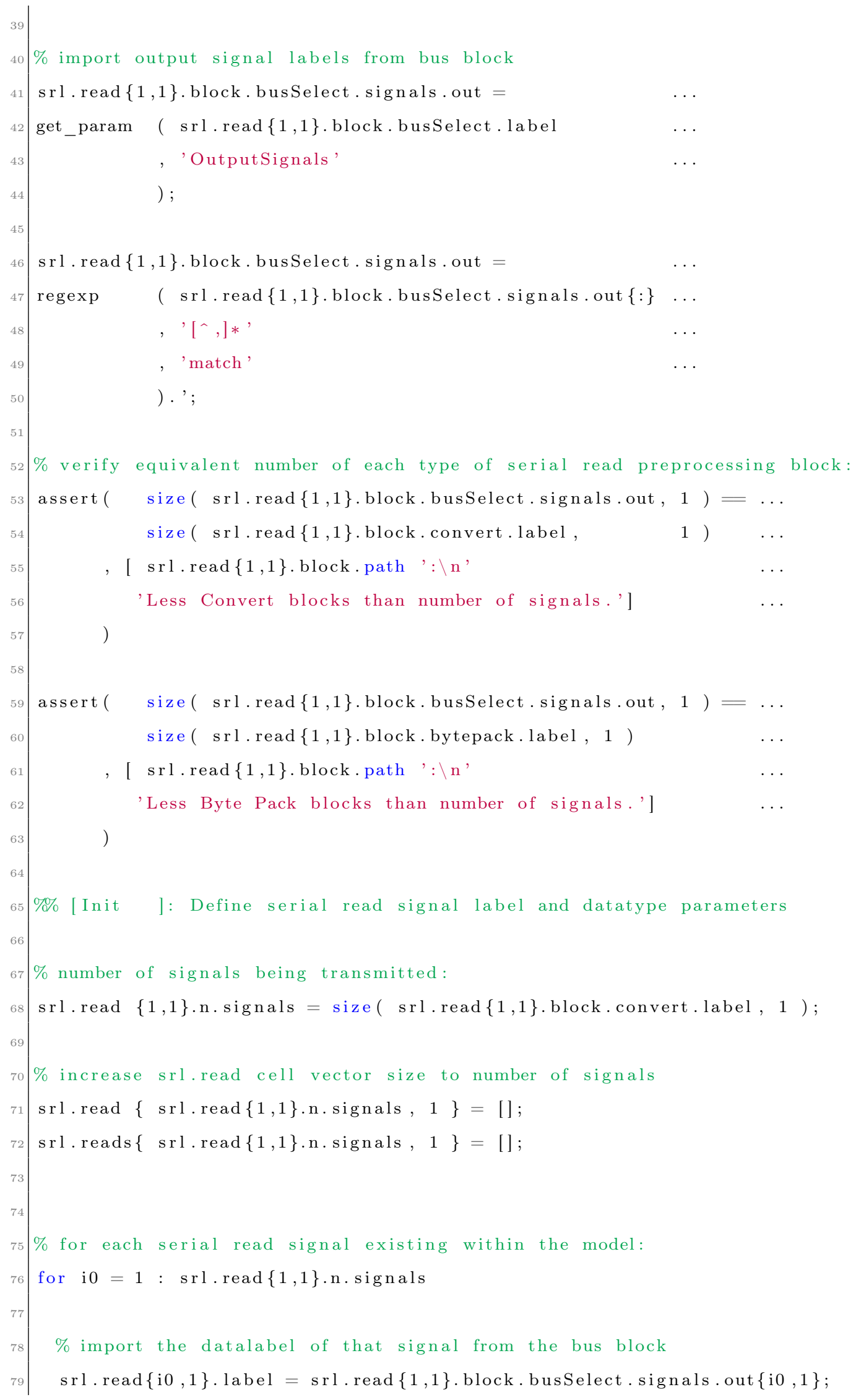




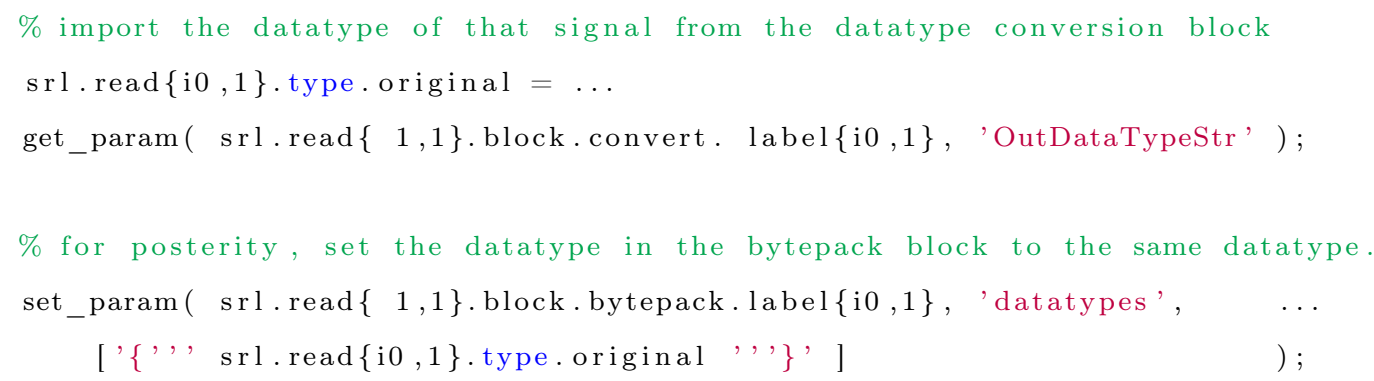




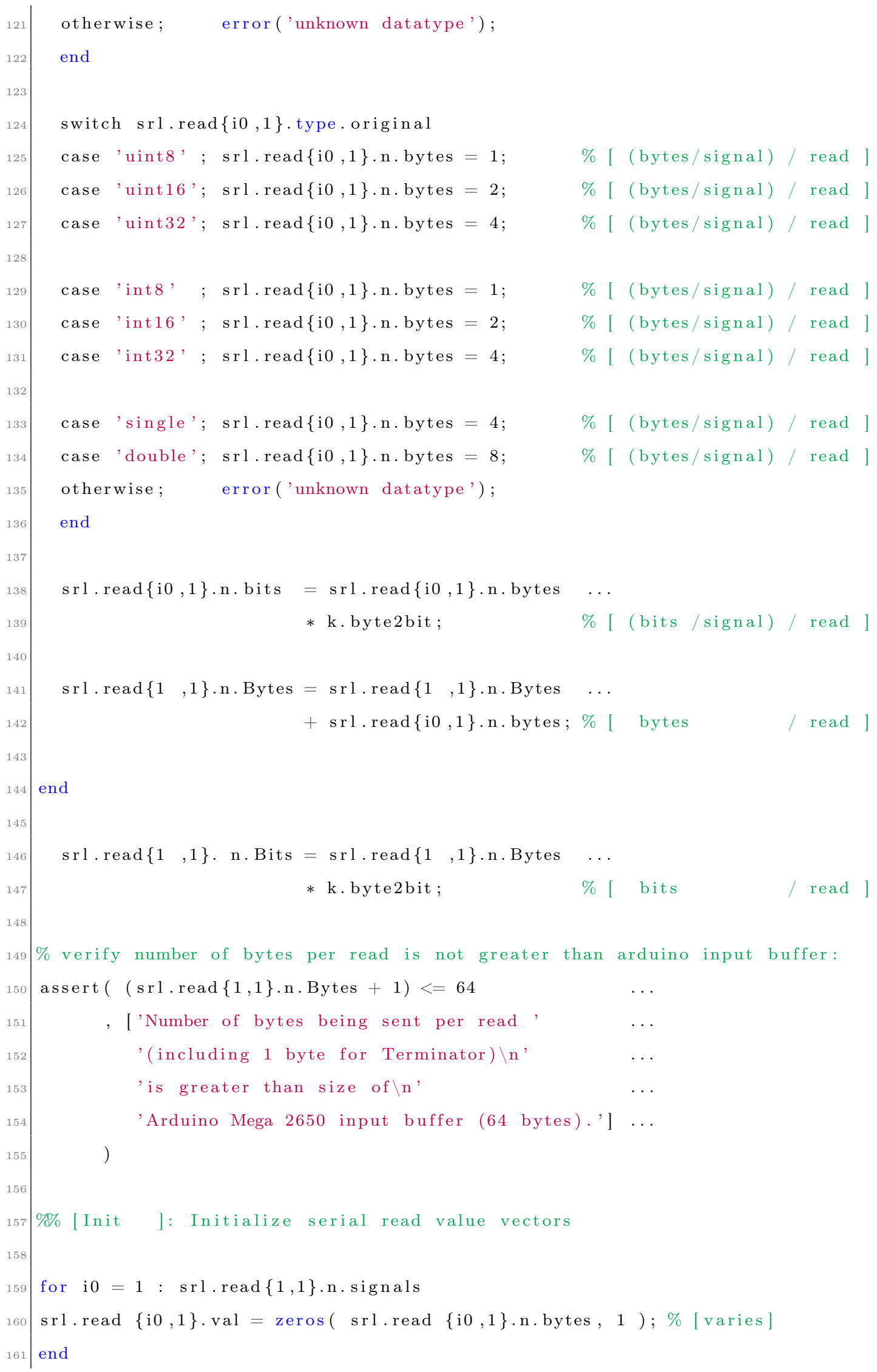




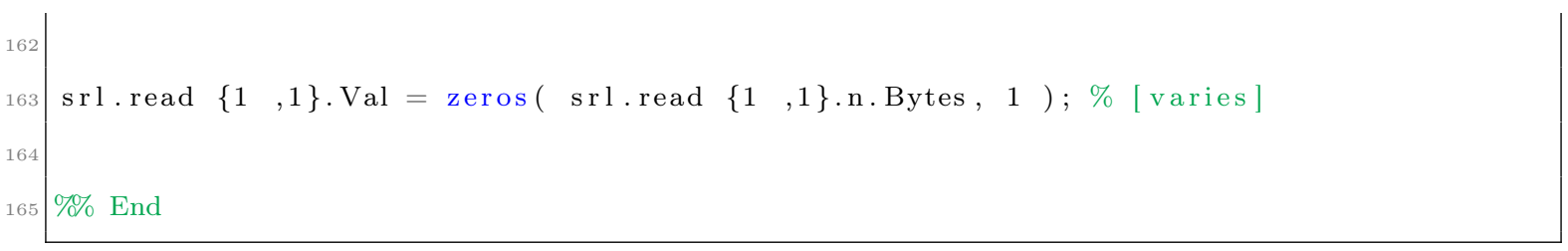

Code Listing A.14: [minseg.m]: Initialization - Serial - Read 
A.1.3.3.3. General

Code Listing A.15: [minseg.m]: Initialization - Serial - General

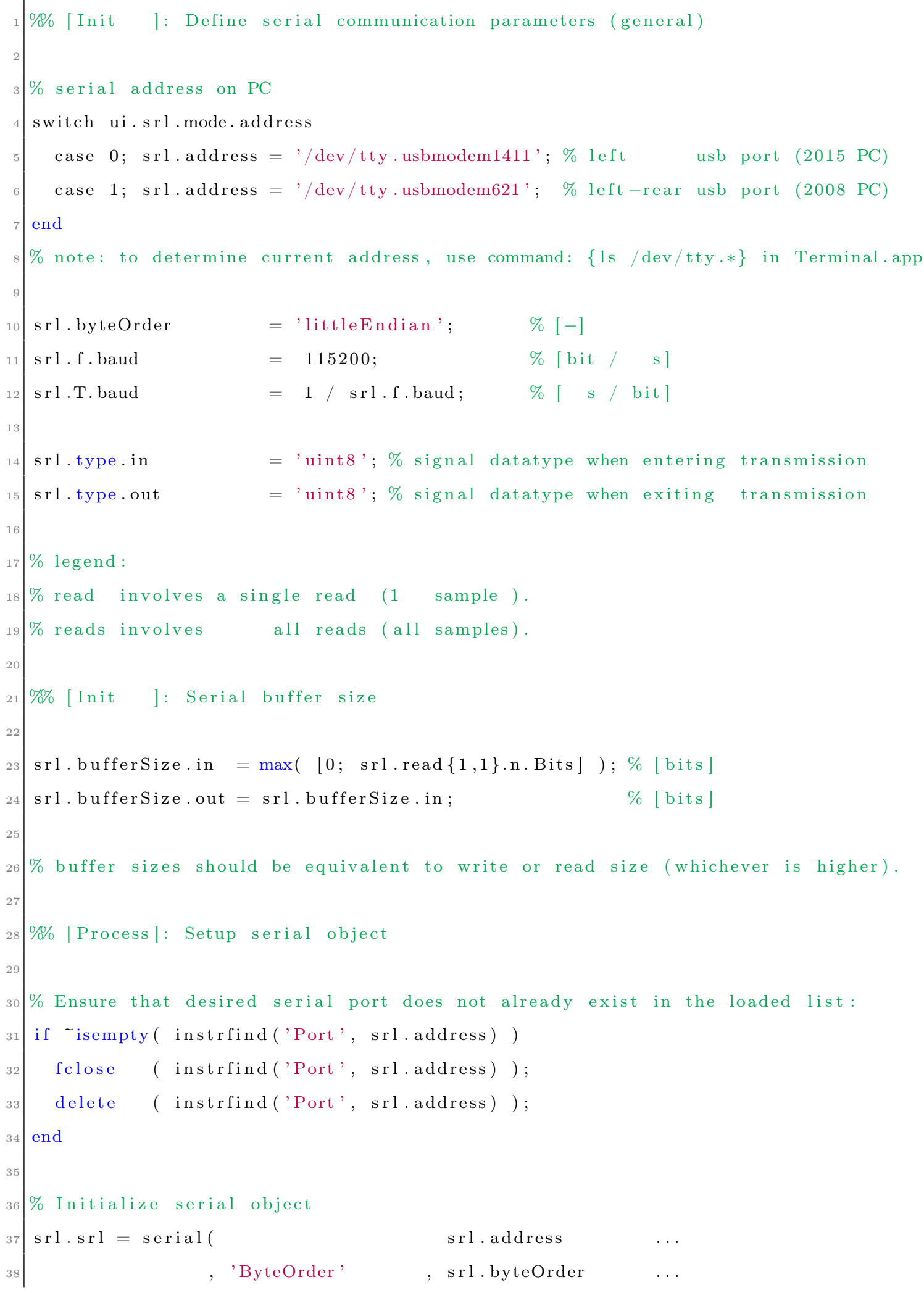




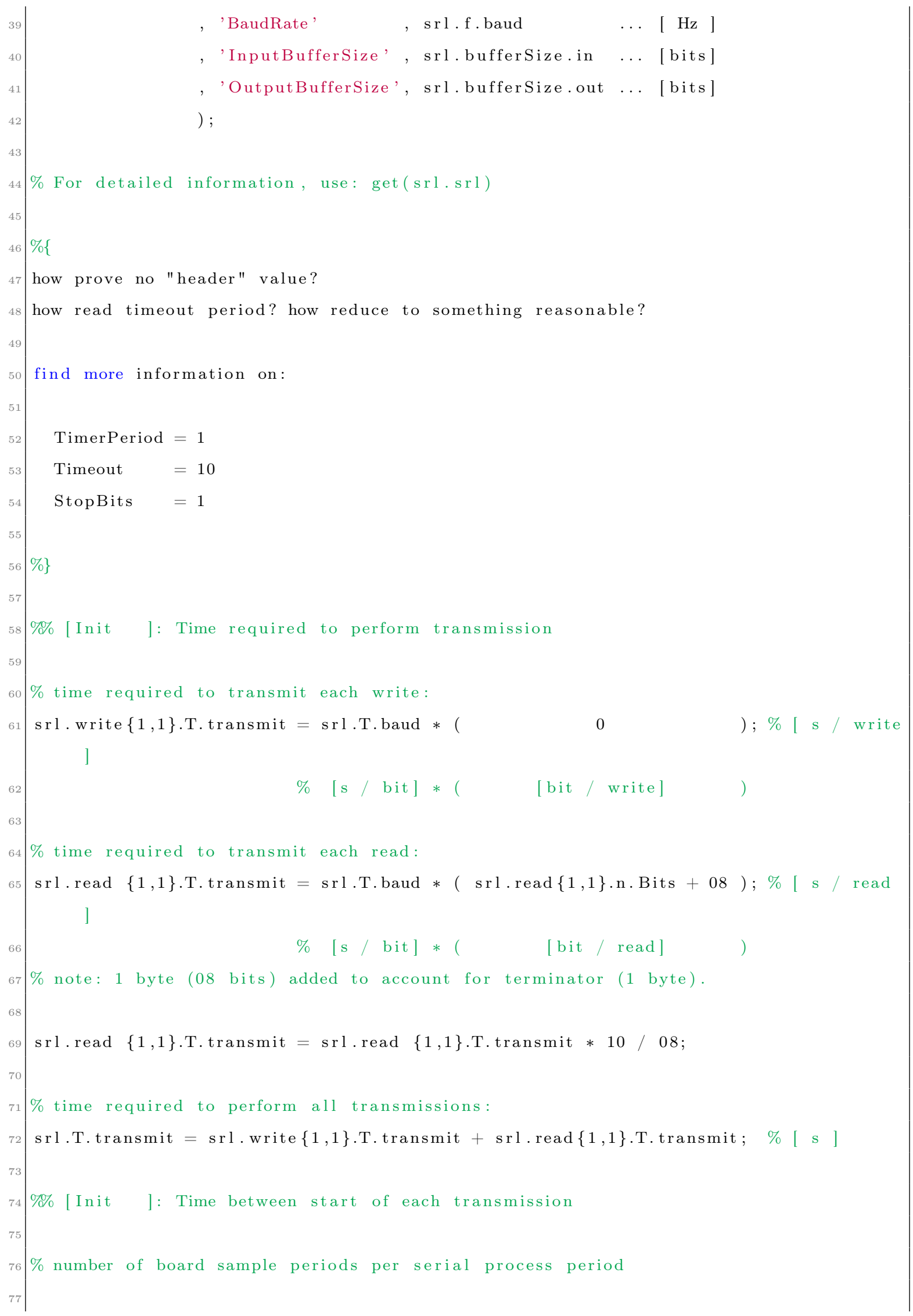




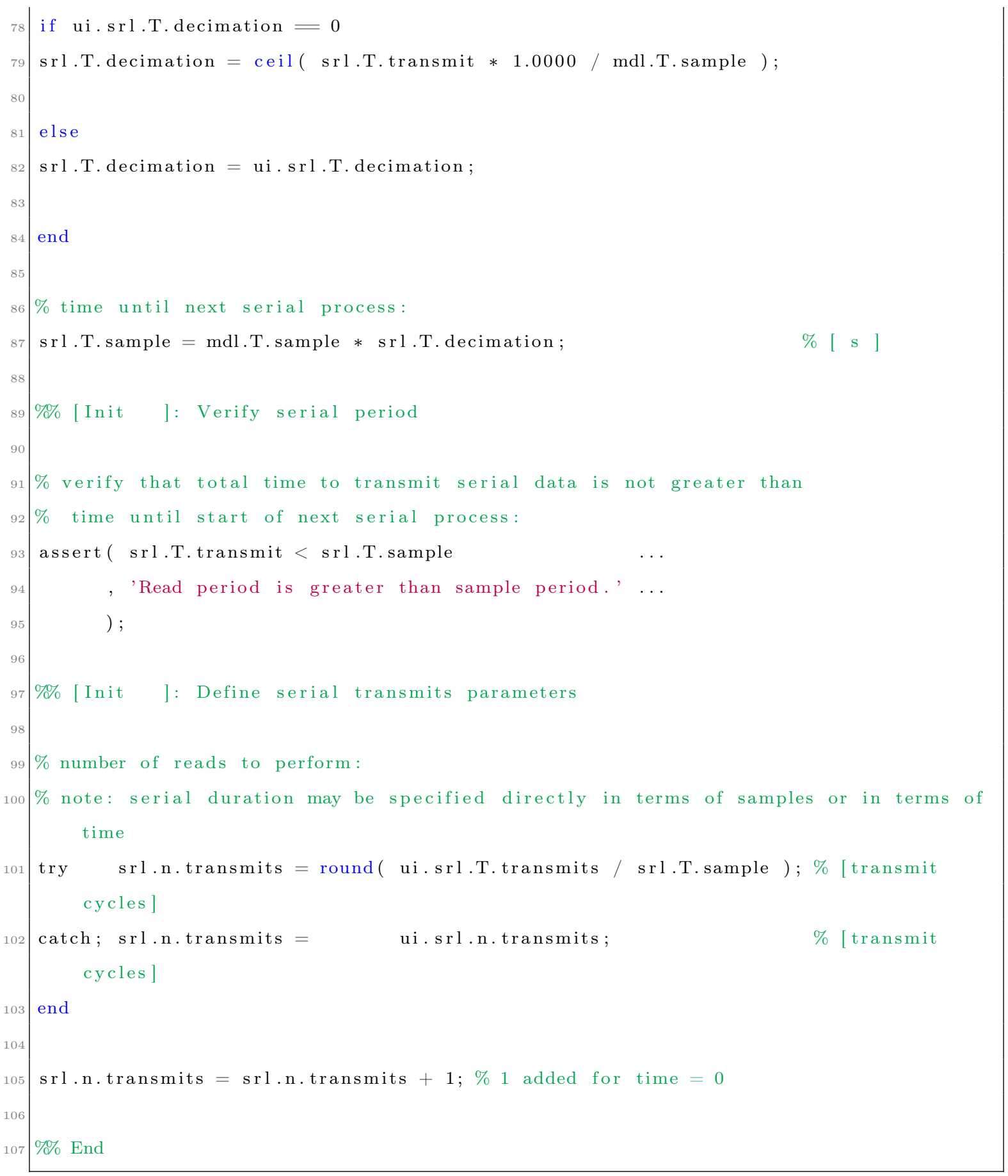

Code Listing A.15: [minseg.m]: Initialization - Serial - General 


\section{A.1.3.3.4. Reads}

Code Listing A.16: [minseg.m]: Initialization - Serial - Reads

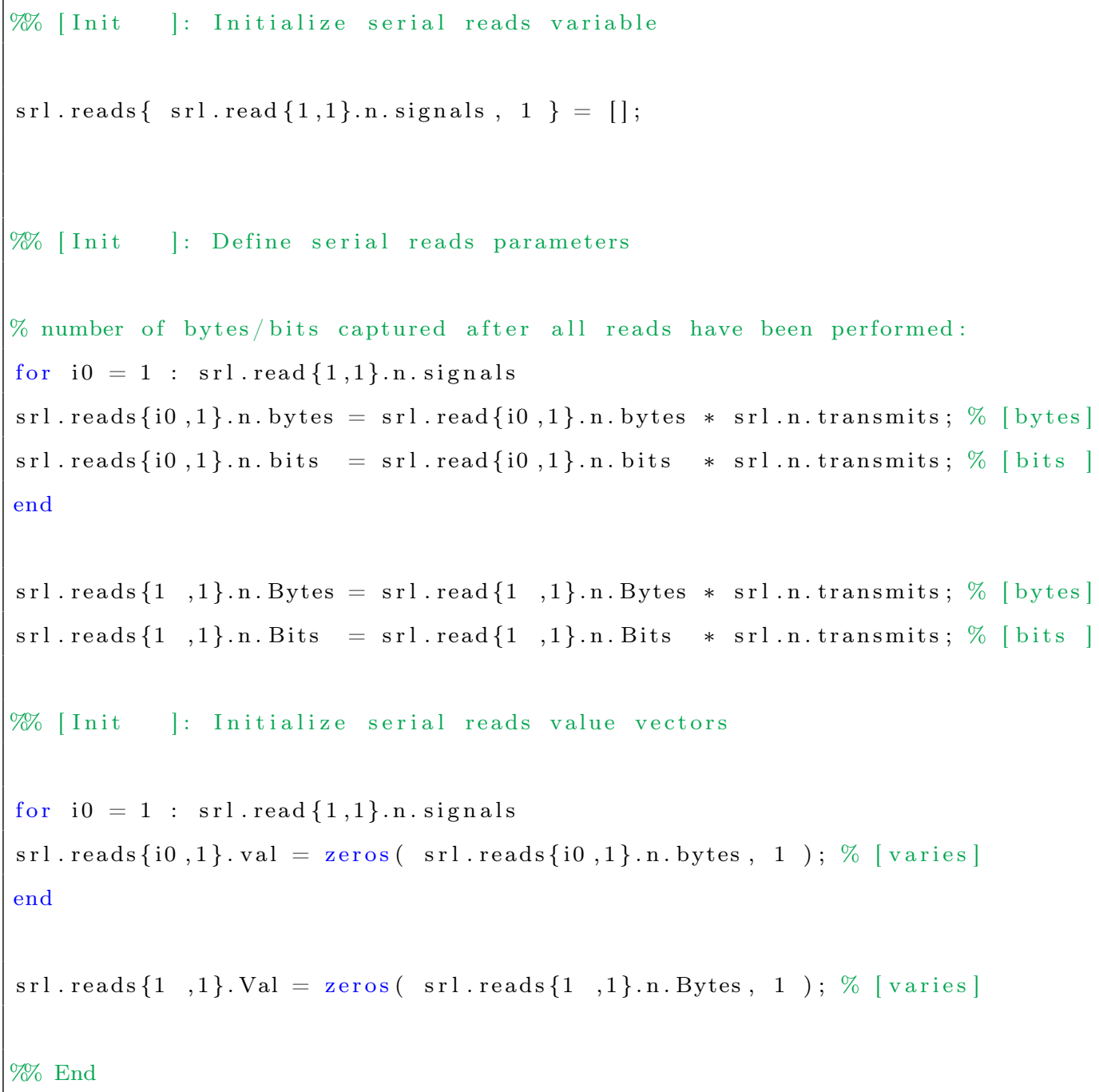

Code Listing A.16: [minseg.m]: Initialization - Serial - Reads 


\section{A.1.3.3.5. Build Parameters}

Code Listing A.17: [minseg.m]: Initialization - Serial - Model Build Parameters

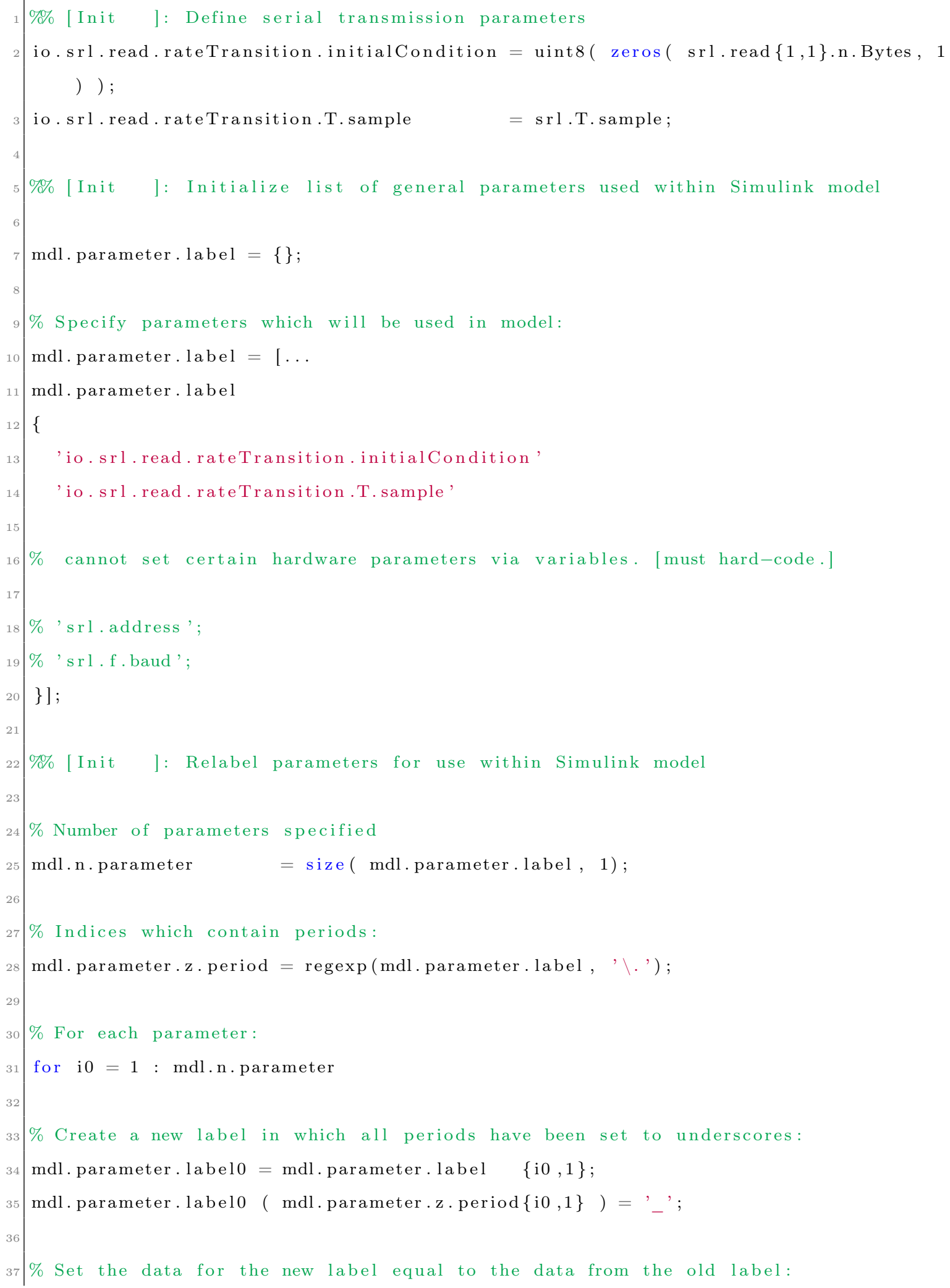




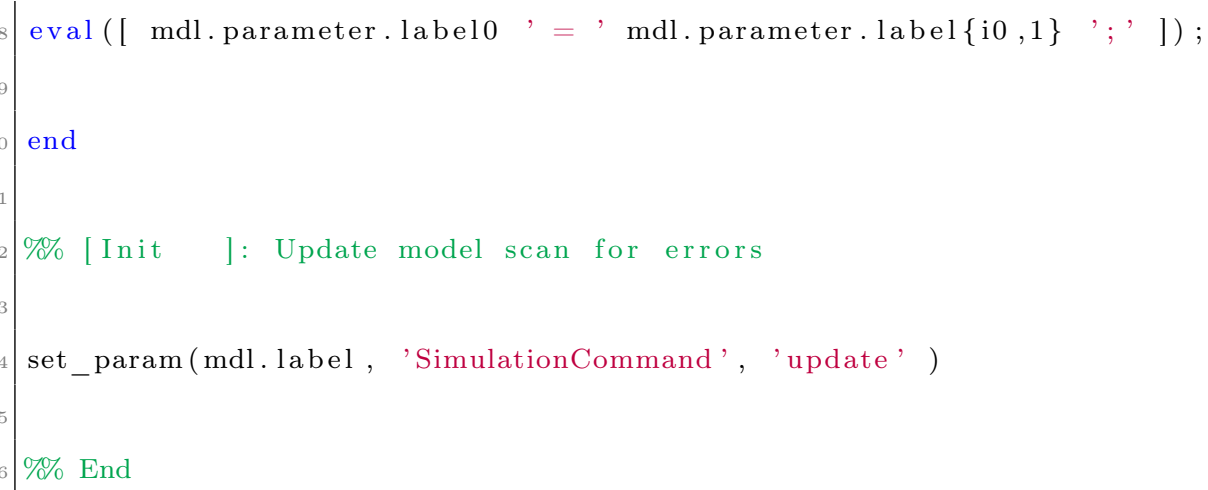

Code Listing A.17: [minseg.m]: Initialization - Serial - Model Build Parameters 
A.1.4. Processing 


\section{A.1.4.1. Build}

Code Listing A.18: [minseg.m]: Processing - Build

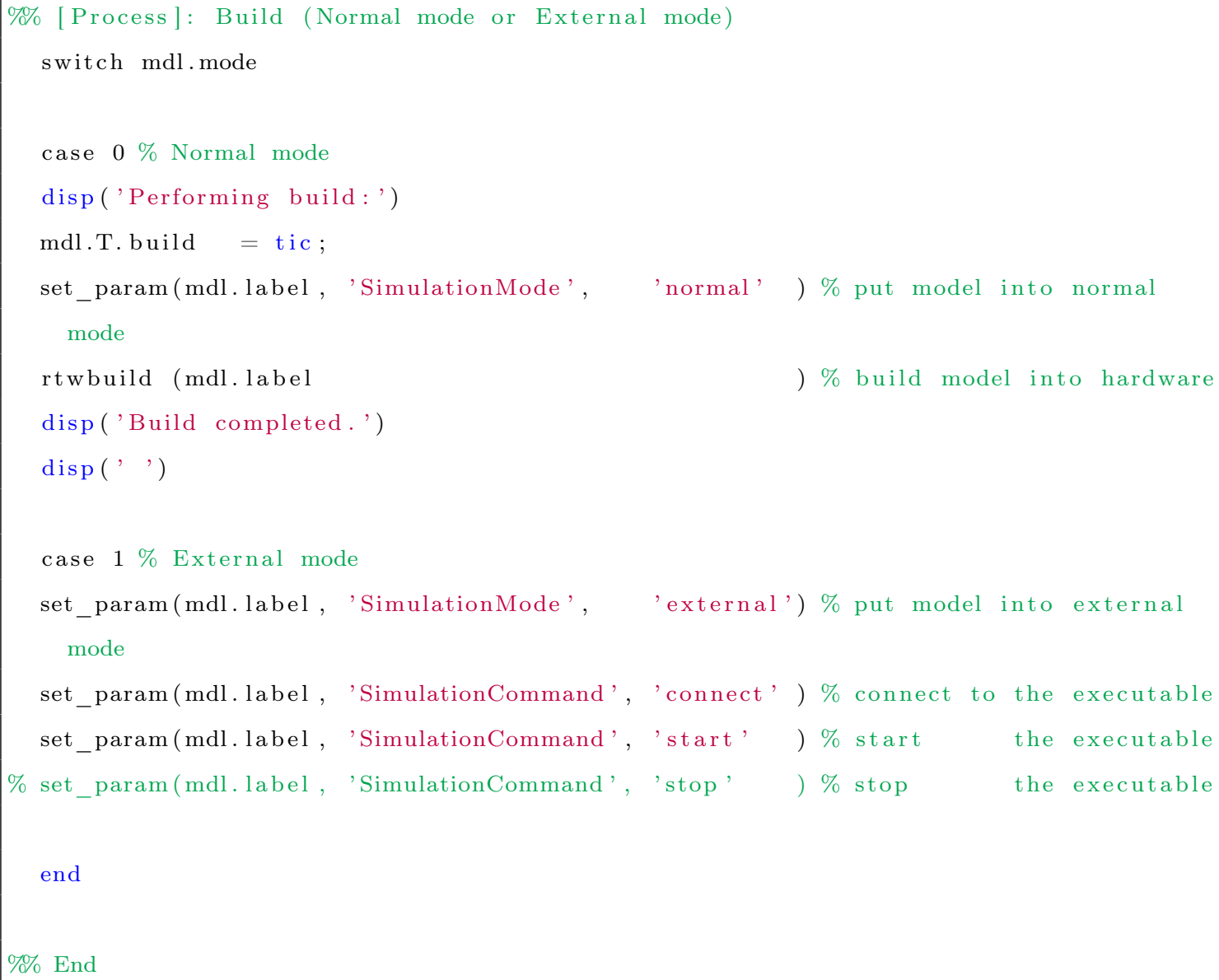

Code Listing A.18: [minseg.m]: Processing - Build 


\section{A.1.4.2. Serial Transmission}

Code Listing A.19: [minseg.m]: Processing - Serial - Transmit

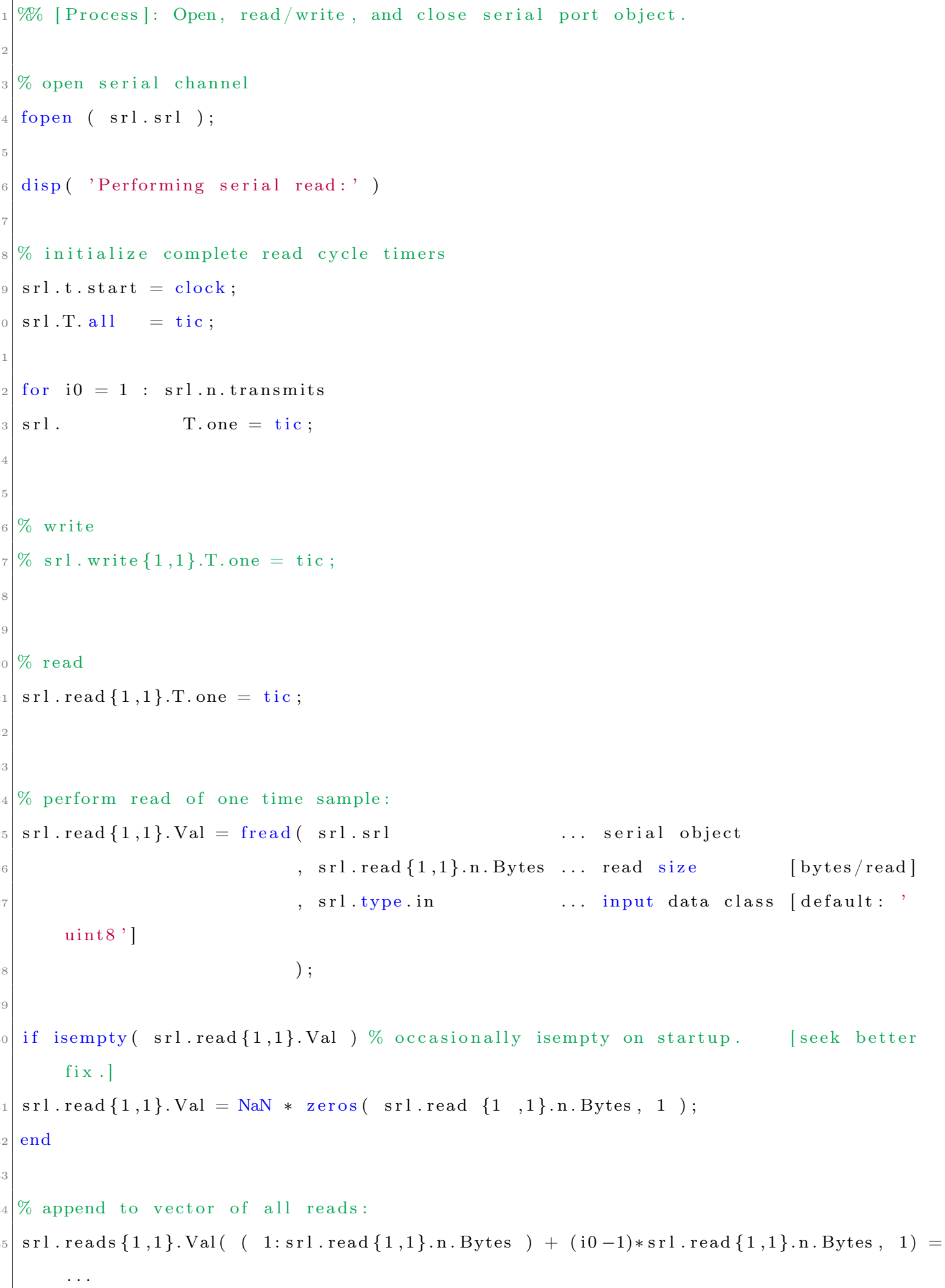




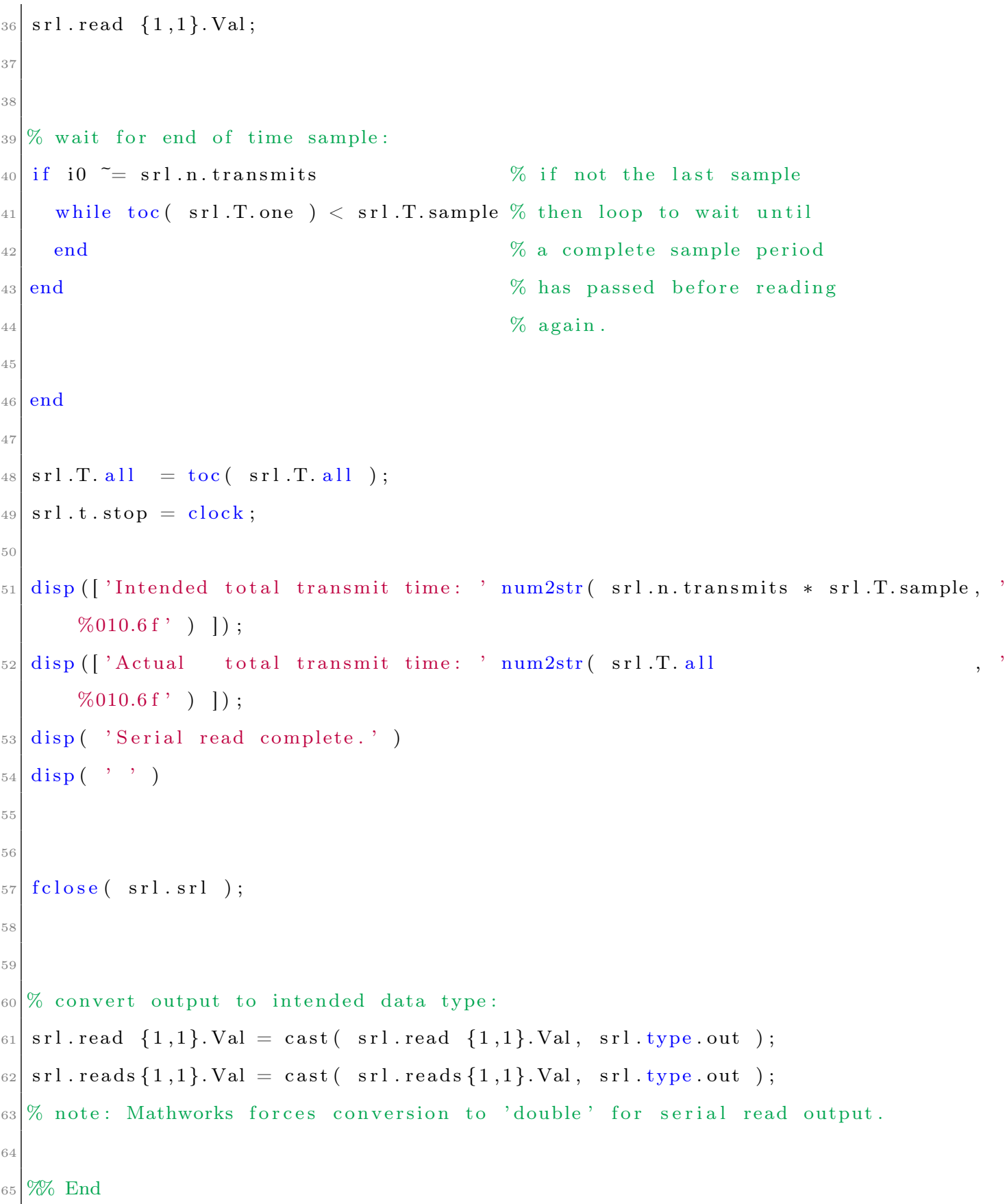

Code Listing A.19: [minseg.m]: Processing - Serial - Transmit 


\section{A.1.4.3. Serial Reads Post-Processing}

Code Listing A.20: [minseg.m]: Processing - Serial - Reads

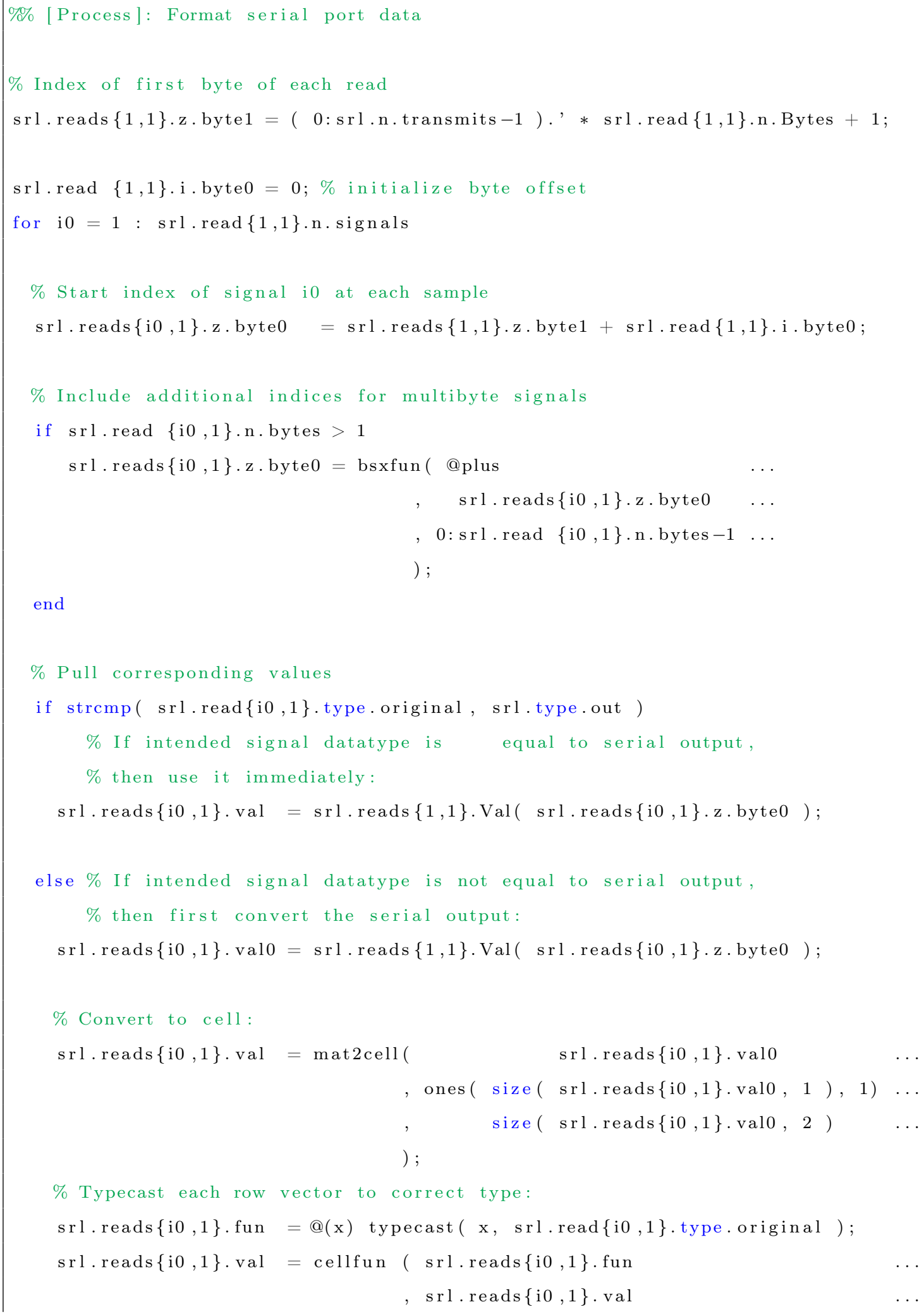




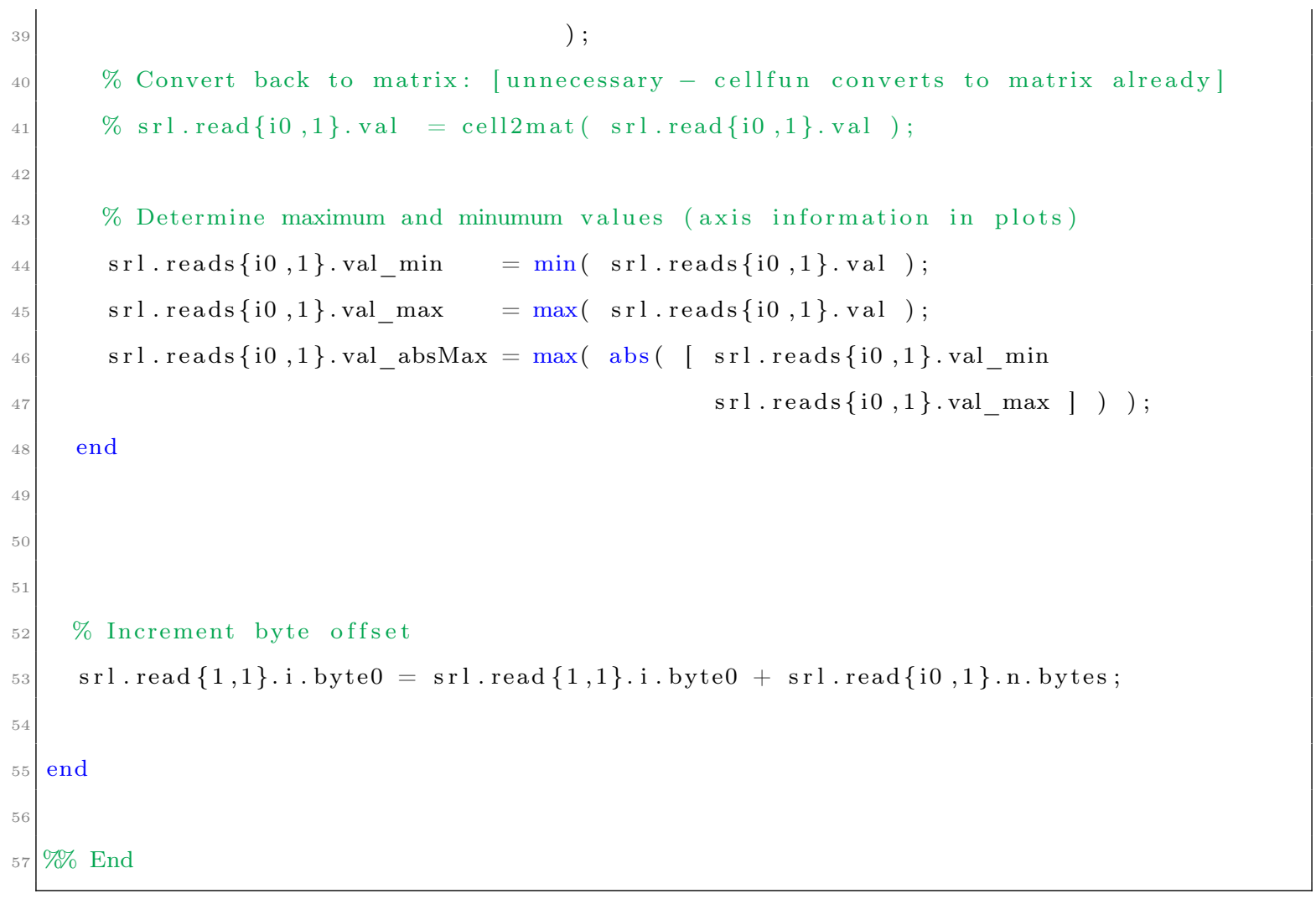

Code Listing A.20: [minseg.m]: Processing - Serial - Reads 
A.1.5. Output 
A.1.5.1. Save

Code Listing A.21: [minseg.m]: Output - Save

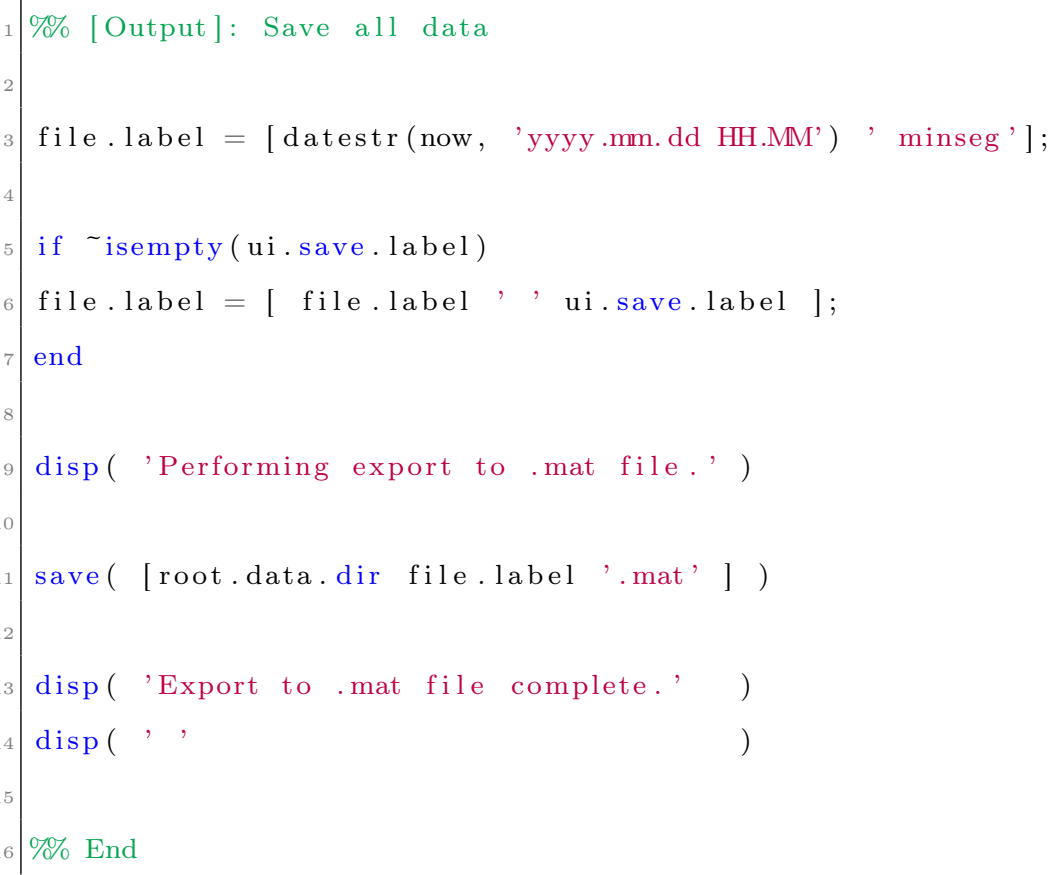

Code Listing A.21: [minseg.m]: Output - Save 


\section{A.1.5.2. Serial Reads Plot}

Code Listing A.22: [minseg.m]: Output - Serial - Reads - Plot

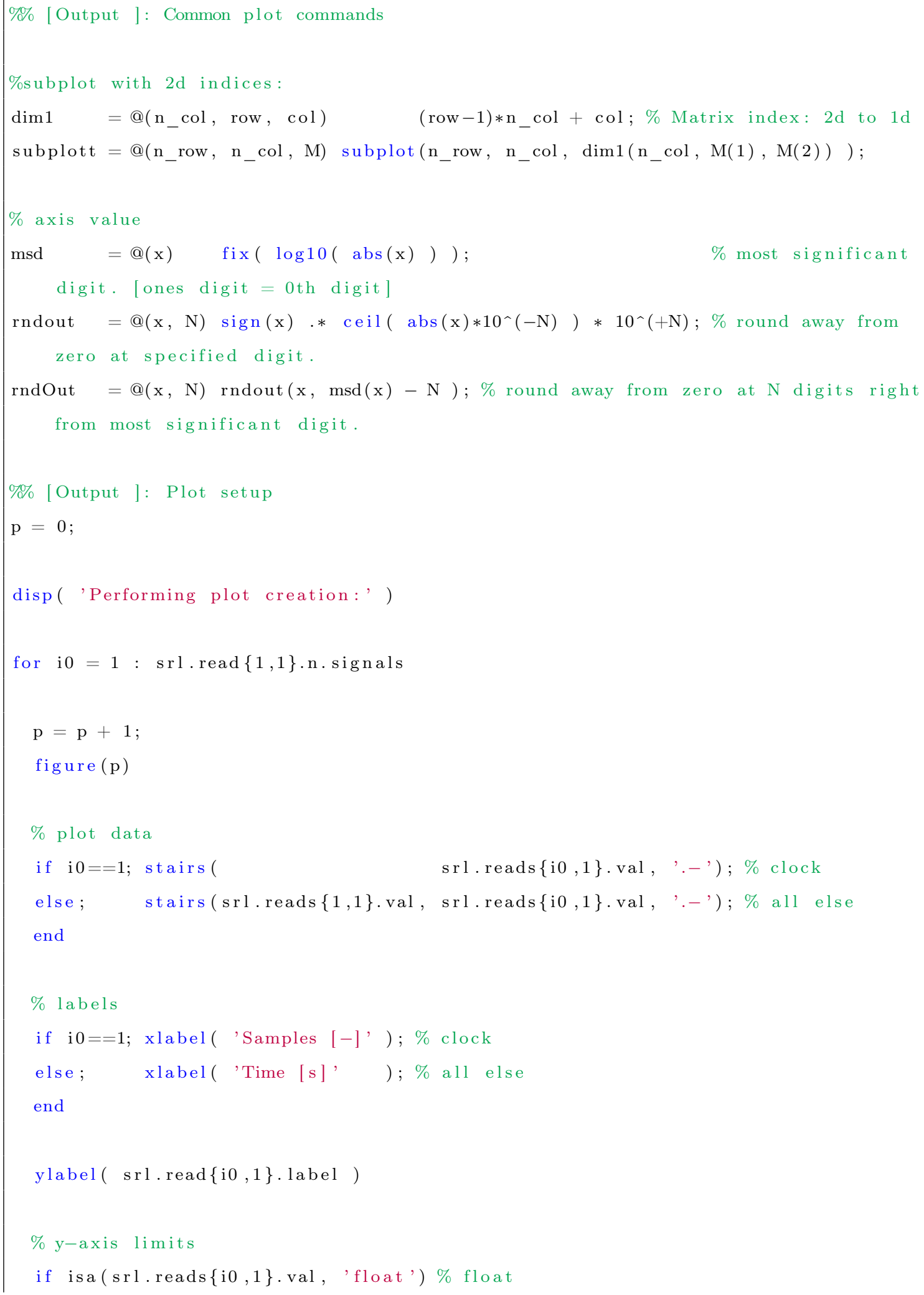




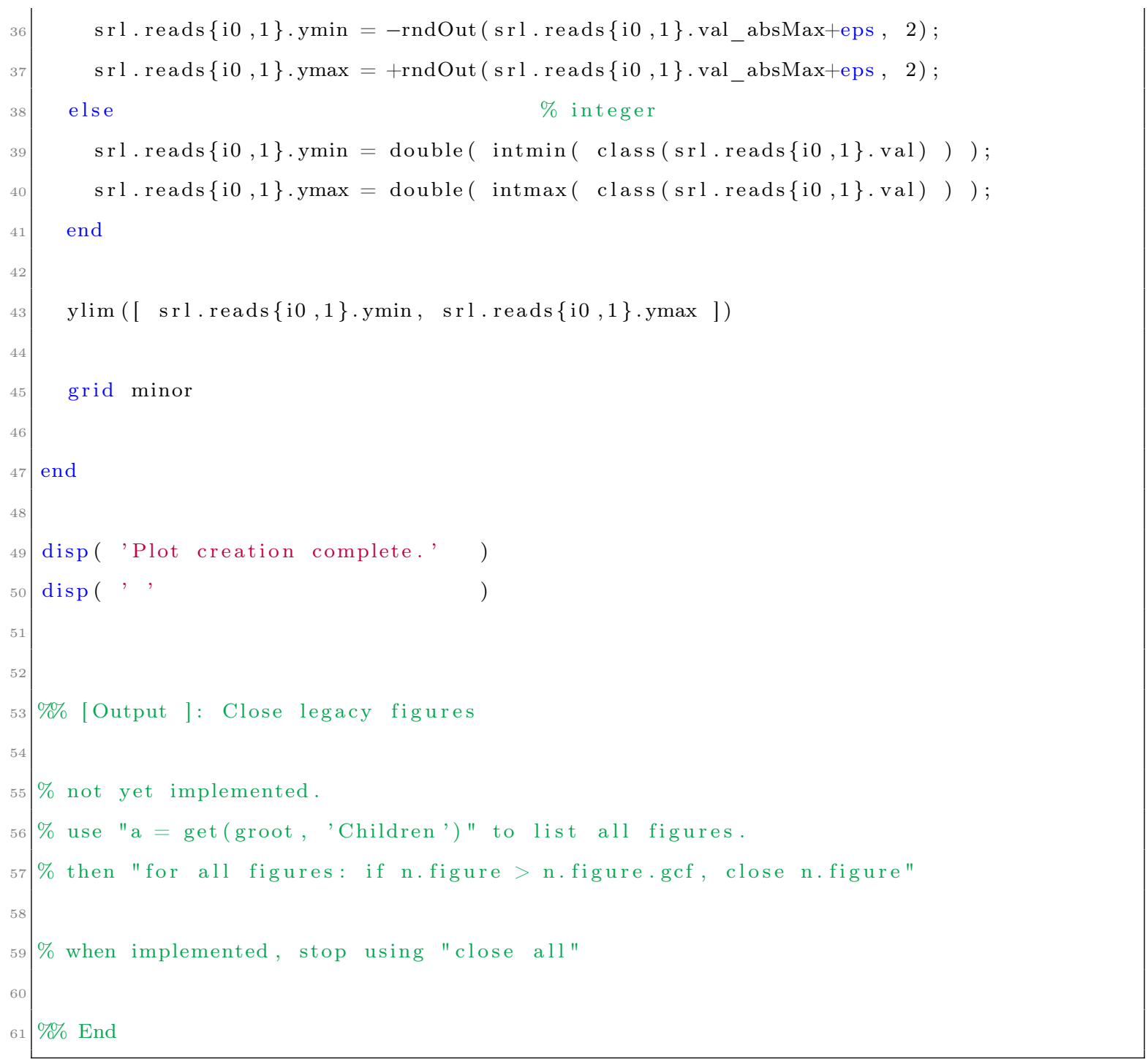

Code Listing A.22: [minseg.m]: Output - Serial - Reads - Plot 


\section{A.1.6. Global Cleanup}

Code Listing A.23: [minseg.m]: Global Cleanup

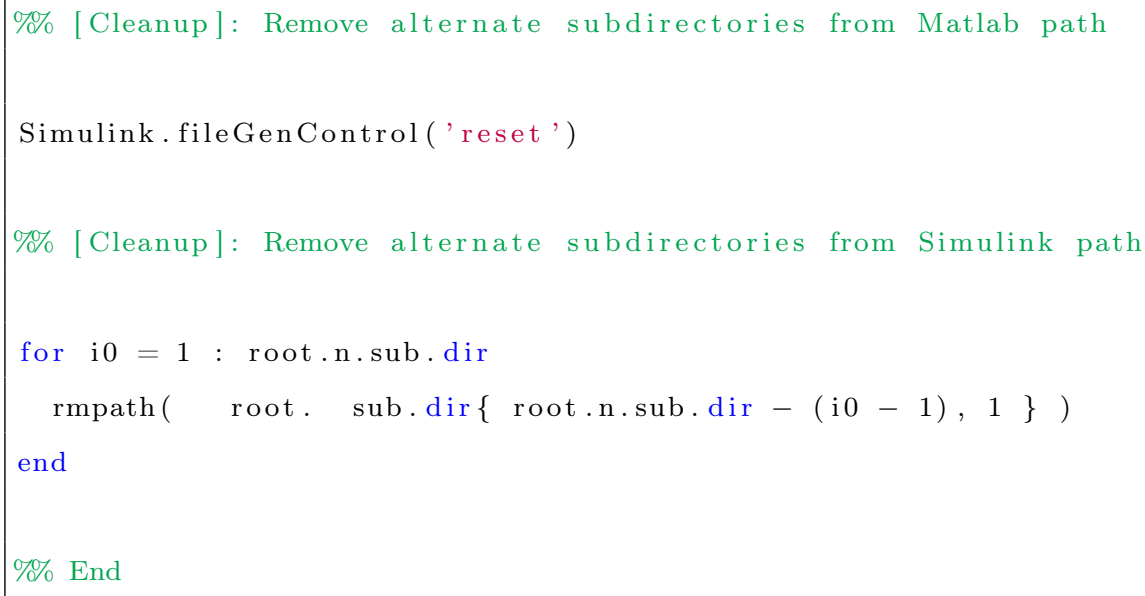

Code Listing A.23: [minseg.m]: Global Cleanup 


\section{Bibliography}

[1] Y. Yamamoto. (May 1, 2009). Nxtway-gs (self-balancing two-wheeled robot) controller design, [Online]. Available: https://www . mathworks . com/matlabcentral/fileexchange/19147nxtway - gs - - self - balancing - two - wheeled - robot - controller - design (visited on $07 / 03 / 2017)$.

[2] R. J. Vaccaro, Digital Control: A State-space Approach, ser. Mcgraw Hill Series in Electrical and Computer Engineering. Mcgraw-Hill College, Jan. 1995, ISBN: 978-0070667815.

[3] Wikipedia. (Jun. 24, 2017). Inverted pendulum, Wikimedia Foundation, [Online]. Available: https://en.wikipedia.org/wiki/Inverted_pendulum (visited on 07/03/2017).

[4] D. Melanson. (Jul. 14, 2016). Researchers create life-saving ubot-5 robot, play dress-up with it, Engadget, [Online]. Available: https : //www . engadget.com/2008/04/17/researcherscreate-life-saving-ubot-5-robot-play-dress-up-with/ (visited on 02/05/2017).

[5] B. Howard and L. Bushnell, "Enhancing linear system theory curriculum with an inverted pendulum robot," in 2015 International Conference on Computer Science and Mechanical Automation (CSMA), IEEE, Hangzhou, China, Oct. 2015. DOI: 10.1109/CSMA . 2015.63.

[6] Mathworks. Arduino programming with matlab and simulink, [Online]. Available: https : //www . mathworks. com/discovery/arduino-programming-matlab-simulink.html (visited on $07 / 03 / 2017)$.

[7] J. Hurst. (Jun. 17, 2016). Rensselaer arduino support package library (rasplib), 1.1, Rensselaer Polytechnic Institute (RPI), [Online]. Available: https : // www . mathworks . com / matlabcentral/fileexchange/62702-rensselaer-arduino-support-package-library-rasplib- (visited on 07/03/2017).

[8] MinSeg. M2v3, [Online]. Available: https : //minseg . com/collections/minseg-kits / products/minsegshield-kit-m2v3-2-dual-drive-segway-and-line-follower (visited on $07 / 03 / 2017)$.

[9] Wikipedia. (Jul. 1, 2017). Inverted pendulum, Wikimedia Foundation, [Online]. Available: https://en.wikipedia.org/wiki/Arduino (visited on 07/03/2017).

[10] jkenny23. (May 9, 2011). Crystal vs. resonator, Arduino, [Online]. Available: https://forum. arduino.cc/index.php?topic=60662.0 (visited on 07/03/2017). 
[11] Arduino Geeks. (Feb. 2, 2017). Arduino mega vs uno compared, Arduino Starter Kits, [Online]. Available: https : / / www . arduinostarterkits . com / reviews / arduino-mega - vs - uno compared/ (visited on $07 / 03 / 2017$ ).

[12] Reichelt Elektronik. Arduino mega: Arduino mega 2560, atmega1280, usb, [Online]. Available: https : //www . reichelt. com/de/en/Single-board-microcontroller/ARDUINO-MEGA/3/ index $\cdot \mathrm{html}$ ?ACTION=3\&GROUPID=6667\&ARTICLE=119696 (visited on 07/03/2017).

[13] Alberto "PighiXXX". (Aug. 4, 2014). Mega, PighiXXX, [Online]. Available: http ://www . pighixxx.com/test/portfolio-items/mega/ (visited on 07/03/2017).

[14] PowerStream. (Jun. 29, 2017). Discharge tests of aa batteries, alkaline and nimh, [Online]. Available: https://www. powerstream.com/AA-tests.htm (visited on 07/03/2017).

[15] Texas Instruments. (Jan. 2015). Sn754410: Quadruple h drivers, [Online]. Available: http: //www.ti.com/product/sn754410?qgpn=sn754410 (visited on 07/03/2017).

[16] P. "Philo" Hurbain. (May 15, 2017). Nxt motor internals, [Online]. Available: http://www . philohome.com/nxtmotor/nxtmotor.htm (visited on 07/03/2017).

[17] Everymac. (May 22, 2017). Apple macbook pro "core i5" 2.7 13" early 2015 specs, [Online]. Available: http://www . everymac.com/systems/apple/macbook_pro/specs/macbook-procore-i5-2.7-13-early-2015-retina-display-specs.html (visited on 07/03/2017).

[18] Mathworks. Previous releases: System requirements and supported compilers, [Online]. Available: https://www . mathworks.com/support/sysreq/previous_releases.html (visited on $07 / 03 / 2017)$.

[19] M. D. Peltier, "Trajectory control of a two-wheeled robot," Master's thesis, University of Rhode Island (URI): Department of Electrical Engineering, Jan. 2012.

[20] F. L. Lewis and V. L. Syrmos, Optimal Control, Second. Wiley-Interscience, Nov. 1995, ISBN: 978-0-471-03378-3.

[21] (Oct. 3, 2016). Lego wheels chart, [Online]. Available: http://wheels.sariel.pl/ (visited on $07 / 03 / 2017)$.

[22] R. P. M. Chan, K. A. Stol, and C. R. Halkyard, "Review of modelling and control of twowheeled robots," Annual Reviews in Control, vol. 37, no. 1, pp. 89-103, Apr. 2013. 
[23] D. R. Jones and K. A. Stol, "Modelling and stability control of two-wheeled robots in lowtraction environments," in Australasian Conference on Robotics and Automation (ACRA) 2010, G. Wyeth and B. Upcroft, Eds., Australian Robotics \& Automation Association (ARAA), Brisbane, Australia, Dec. 2010.

[24] O. Jamil, M. Jamil, Y. Ayaz, and A. Khubab, "Modeling, control of a two-wheeled selfbalancing robot," in 2014 International Coneference on Robotics and Emerging Allied Technologies in Engineering (iCREATE), IEEE, Islamabad, Pakistan, Apr. 2014. DOI: 10.1109/ ICREATE. 2014.6828364.

[25] R. J. Vaccaro, "An optimization approach to the pole-placement design of robust linear multivariable control systems," in 2014 American Control Conference (ACC), ACC, Portland, OR, USA, Jun. 2014. DOI: 10.1109/ACC. 2014.6858987.

[26] Y. Gong, H. Ma, and X. Wu, "Research on control strategy of two-wheeled self-balancing robot," in 2015 International Conference on Computer Science and Mechanical Automation (CSMA), IEEE, Hangzhou, China, Oct. 2015. DOI: 10.1109/CSMA . 2015.63.

[27] R. J. Vaccaro, Personal Interview, University of Rhode Island (URI): Department of Electrical Engineering, Jan. 23, 2017.

[28] MinSeg. Homepage, [Online]. Available: https://minseg.com/ (visited on 07/03/2017).

[29] Mathworks. Homepage, [Online]. Available: https : / / www . mathworks . com/ (visited on $07 / 03 / 2017)$.

[30] A. De Luca, G. Oriolo, and M. Vendittelli, "Control of wheeled mobile robots: An experimental overview," in Ramsete: Articulated and Mobile Robotics for Services and Technologies. Berlin, Heidelberg: Springer Berlin Heidelberg, 2001, pp. 181-226, ISBN: 978-3-540-45000-9. DOI: 10. 1007/3-540-45000-9_8. [Online]. Available: http://dx .doi .org/10.1007/3-540-45000$9 \_8$.

[31] M. R. Bageant, Balancing a two-wheeled segway robot, Undergraduate Thesis, Oct. 2011.

[32] A. R. da Silva Jr, "Design and control of a two-wheeled robotic walker," Master's thesis, University of Massachusettes (UMass): Department of Mechanical and Industrial Engineering, May 2014. 